\title{
Low-order stochastic modelling of low-frequency motions in reflected shock-wave/boundary-layer interactions
}

\author{
EMILE TOUBER $†$ AND NEIL D. SANDHAM \\ Aerodynamics and Flight Mechanics Research Group, School of Engineering Sciences, \\ University of Southampton, University Road, Southampton, SO17 1BJ, UK
}

(Received 31 December 2009; revised 8 July 2010; accepted 7 November 2010)

A combined numerical and analytical approach is used to study the low-frequency shock motions observed in shock/turbulent-boundary-layer interactions in the particular case of a shock-reflection configuration. Starting from an exact form of the momentum integral equation and guided by data from large-eddy simulations, a stochastic ordinary differential equation for the reflected-shock-foot low-frequency motions is derived. During the derivation a similarity hypothesis is verified for the streamwise evolution of boundary-layer thickness measures in the interaction zone. In its simplest form, the derived governing equation is mathematically equivalent to that postulated without proof by Plotkin (AIAA J., vol. 13, 1975, p. 1036). In the present contribution, all the terms in the equation are modelled, leading to a closed form of the system, which is then applied to a wide range of input parameters. The resulting map of the most energetic low-frequency motions is presented. It is found that while the mean boundary-layer properties are important in controlling the interaction size, they do not contribute significantly to the dynamics. Moreover, the frequency of the most energetic fluctuations is shown to be a robust feature, in agreement with earlier experimental observations. The model is proved capable of reproducing available lowfrequency experimental and numerical wall-pressure spectra. The coupling between the shock and the boundary layer is found to be mathematically equivalent to a first-order low-pass filter. It is argued that the observed low-frequency unsteadiness in such interactions is not necessarily a property of the forcing, either from upstream or downstream of the shock, but an intrinsic property of the coupled system, whose response to white-noise forcing is in excellent agreement with actual spectra.

Key words: compressible boundary layers, low-dimensional models, shock waves

\section{Introduction}

In recent years, shock-wave/turbulent boundary-layer interactions (SBLI) have received renewed interest, thanks to considerable progress in experimental and computational techniques. A principal concern is the occurrence of energetically significant low-frequency shock motions, which in turn can lead to undesirable unsteady pressure loads in practical aerospace applications. Recent experimental observations (see Dupont, Haddad \& Debiève 2006; Piponniau et al. 2009; Polivanov, Sidorenko \&

$\dagger$ Present address: Department of Aeronautics, Imperial College London, South Kensington Campus, London SW7 2AZ, UK. Email address for correspondence: e.touber@imperial.ac.uk 
Maslov 2009; Ringuette et al. 2009) and numerical studies (see Wu \& Martin 2008; Garnier 2009; Priebe, Wu \& Martin 2009; Touber \& Sandham 2009a,b) are in agreement that the pressure fluctuations are broadband in nature and centred around a frequency which is one to two orders of magnitude smaller than the characteristic frequency of the incoming boundary layer $\bar{u}_{1} / \delta_{0}$, where $\bar{u}_{1}$ is the upstream potentialflow velocity and $\delta_{0}$ is the upstream $99 \%$ boundary-layer thickness.

The physical mechanisms at the origin of the low-frequency shock motions are not currently understood but a number of tentative explanations have been proposed, usually falling into one of two categories. The first relates the low-frequency motions to specific events or flow structures from the upstream turbulent boundary layer, whereas the second looks for causal mechanisms within the interaction itself (i.e. downstream of the shock). In both cases, the difficulty resides in identifying a mechanism that can span time scales of the order of $10^{1} \delta_{0} / \bar{u}_{1}$ to $10^{2} \delta_{0} / \bar{u}_{1}$. With respect to the second category, a variety of mechanisms have been proposed. Piponniau et al. (2009) argue that the mass-entrainment time scale associated with the separation bubble and the developing mixing layer above it scales with the shock-motion time scales of interest. They suggest that the main controlling parameter is the compressible mixing-layer spreading rate, provided that a separation bubble is formed. Under such conditions, they indicate that the geometry of the flow configuration (i.e. compression corner or shock reflection) has little influence on the low-frequency dynamics. On the basis of direct numerical simulation (DNS) of a shock-reflection configuration, Pirozzoli \& Grasso (2006) suggest that the low-frequency shock motions could result from an acoustic resonance similar to Rossiter modes in cavity flows, where the shear-layer at the separation-bubble interface acts as the amplifier, the incident-shock tip as the source point of the acoustic field (produced by shock/vortex interactions), with feedback via the region of subsonic flow and receptivity of the shear layer to the acoustic field. It is unclear whether or not the low-frequency tones described in their results originate from sufficiently long data samples, knowing that in practice the oscillations are broadband. Yet another suggestion has been to relate the lowfrequency motions with an intrinsic hydrodynamic instability (see Robinet 2007 and Touber \& Sandham 2009b, for details).

Returning to the first category of mechanisms, the experimental work of Ganapathisubramani, Clemens \& Dolling $(2007,2009)$ is of special interest, since the authors find direct correlations between $O\left(50 \delta_{0}\right)$-long coherent structures in the incoming boundary layer and the shock motions. While the shock is undoubtedly affected by the passage of low- or high-speed streaks, as for instance evidenced by the tomographic particle image velocimetry study of Humble et al. (2009), the success of the mechanism studied by Ganapathisubramani et al. (2009) in explaining the low-frequency shock motions depends on the existence of sufficiently long streaky structures. Touber \& Sandham (2009b) performed large-eddy simulation (LES) of a shock-reflection configuration where special care was devoted to the inflow conditions to prevent the development of coherent structures more than $10 \delta_{0}$ long. Long data samples were acquired clearly establishing that, although the upstream boundary layer was deprived of very long coherent structures (i.e. more than $10 \delta_{0}$ long), the low-frequency shock motions could still be observed. This result does not mean that if present, long coherent structures as described by Ganapathisubramani et al. (2007) do not contribute to the low-frequency shock motions but it is an indication that they are not necessary for the underlying low-frequency SBLI dynamics.

In the authors' view, the variety of the mechanisms proposed in the literature, together with the subsequent debate about the merits of one approach relative to 
another, is symptomatic of the difficulty one has in identifying and then separating individual events from a (supposedly) nonlinear (chaotic) system, where actual causal events may well be impossible to detect. Instead of attempting to check the relevance of one assumed mechanism against numerical/experimental data with the inherent complexity of extracting this from fully turbulent flow, it could be more useful to identify the properties of the dynamical system arising from the coupling between the shock and the boundary layer. To some extent, this is the approach followed by Plotkin (1975), who postulated that the shock displacement was obeying a first-order stochastic ordinary differential equation (ODE) with an associated characteristic time scale. Plotkin has shown that such a mathematical model is capable of reproducing the wall-pressure low-frequency spectrum. To the best of the authors' knowledge, this interesting point has only been verified in two subsequent papers by Poggie \& Smits (2001, 2005). Two main reasons why Plotkin's model has not been widely adopted are: (i) it is a postulate and therefore lacks a physical basis for its ability to reproduce experimental wall-pressure spectra; (ii) it is impractical since the key parameter, the characteristic time scale of the ODE, needs to be determined a posteriori from existing data. Arguably, (ii) may be seen as a corollary of (i).

Nevertheless, it is rather intriguing that a relatively simple ODE is capable of reproducing the low-frequency spectra. The mathematical implications of this observation have been considered only at a superficial level. For example, one can read that Plotkin's model is a mathematical explanation of how relatively broadband perturbations, caused by the incoming turbulence, can lead to relatively low-frequency motions; or that it assumes that the restoring mechanism ensuring the shock stability is linear. However, there are more subtle implications. First, the analytical expression given by Plotkin for the spectrum is based on the response to white noise, meaning that the model does not assume as an input a turbulent signal but instead one which is equally composed of high and low frequencies. Second, while it is true that the postulated governing equation is linear, it is possible that the time constant associated with the restoring mechanism already incorporates nonlinear interactions between a velocity fluctuation and the coupled shock/boundary-layer system. This latter point is clearly indicated by Poggie \& Smits (2001).

The present paper aims at deriving an equation describing the shock lowfrequency motions, in the spirit of Plotkin's pioneering work, but from a completely different approach. The case of a shock-impingement configuration is chosen but this work could be extended to compression-ramp flows in the future. A combined LES/analytical approach is used, where the LES results are extensively employed to support and guide each step of the derivations. This work is organised as follows. Section 2 presents the numerical procedure used to generate the LES database. Section 3 describes the conditionally averaged LES data, which will be constantly used throughout the paper. We then move on to the derivations of the shock-foot dynamical equation in $\S 4$, the constituents of which are then modelled in $\S 5$. Section 6 summaries the final closed form of the model and presents some solutions of interest. Finally, $\S 7$ discusses the low-frequency shock motions in the light of the model. More mathematical aspects have been given in appendices.

\section{Numerical procedure for the large-eddy simulations}

\subsection{Governing equations and numerical approach}

The following filtered dimensionless three-dimensional compressible Navier-Stokes equations (expressed in conservative form) are solved. They are composed of one 
continuity equation, three momentum equations and the energy equation:

$$
\begin{aligned}
& \frac{\partial\langle\rho\rangle}{\partial t}+\frac{\partial\langle\rho\rangle \breve{u}_{i}}{\partial x_{i}}=0 \\
& \frac{\partial\langle\rho\rangle \breve{u}_{i}}{\partial t}+\frac{\partial\langle\rho\rangle \breve{u}_{i} \breve{u}_{j}}{\partial x_{j}}+\frac{\partial\langle p\rangle}{\partial x_{i}}-\frac{1}{R e} \frac{\partial\left\langle\tau_{i j}\right\rangle}{\partial x_{j}} \approx-\frac{\partial \sigma_{i j}}{\partial x_{j}}, \\
& \frac{\partial\left\langle E_{t}\right\rangle}{\partial t}+\frac{\partial\left[\left\langle E_{t}\right\rangle+\langle p\rangle\right] \breve{u}_{j}}{\partial x_{j}}-\frac{1}{\operatorname{Re}} \frac{\partial\left\langle\tau_{i j}\right\rangle \breve{u}_{i}}{\partial x_{j}}+\frac{1}{(\gamma-1) \operatorname{RePr} M_{1}^{2}} \frac{\partial}{\partial x_{j}}\left[\langle\mu\rangle \frac{\partial\langle T\rangle}{\partial x_{j}}\right] \\
& \approx-\breve{u}_{i} \frac{\partial \sigma_{i j}}{\partial x_{j}}-\frac{1}{(\gamma-1) M_{1}^{2}} \frac{\partial}{\partial x_{j}}\left[\langle\rho\rangle \Psi_{j}\right],
\end{aligned}
$$

where $\rho$ is the fluid density, $u_{i}$ is the instantaneous velocity vector, $p$ is the pressure, $T$ is the temperature and $t$ is the time. The subscript 1 denotes that the quantity is taken to be in the potential flow upstream of the interaction. The streamwise, wall-normal and spanwise directions are denoted by $x, y$ and $z$, respectively. The resolved equation of state, the resolved total energy/temperature relation and the resolved viscous shear-stress relations are, respectively,

$$
\begin{aligned}
\langle p\rangle & =\frac{1}{\gamma M_{1}^{2}}\langle\rho\rangle\langle T\rangle, \\
\langle T\rangle & =\gamma(\gamma-1) M_{1}^{2}\left[\frac{\left\langle E_{t}\right\rangle}{\langle\rho\rangle}-\frac{1}{2} \breve{u}_{i} \breve{u}_{i}\right], \\
\left\langle\tau_{i j}\right\rangle & =\langle\mu\rangle\left(\frac{\partial \breve{u}_{j}}{\partial x_{i}}+\frac{\partial \breve{u}_{i}}{\partial x_{j}}-\frac{2}{3} \delta_{i j} \frac{\partial \breve{u}_{k}}{\partial x_{k}}\right) .
\end{aligned}
$$

The resolved dynamic viscosity $\langle\mu\rangle$ is related to the resolved temperature assuming either a power law or Sutherland's law (depending on the case):

$$
\begin{aligned}
& \langle\mu\rangle=\langle T\rangle^{\Omega}, \\
& \langle\mu\rangle=\langle T\rangle^{3 / 2} \frac{1+C_{s}}{\langle T\rangle+C_{s}},
\end{aligned}
$$

where $C_{s}=S / \bar{T}_{1}^{\star}$, with $S$ being the Sutherland temperature and $\bar{T}_{1}^{\star}$ being the mean upstream free-stream dimensional temperature. In the present calculations, $C_{s}$ is 0.76 while $\Omega$ is set to 0.67 .

The $\langle\cdot\rangle$ and $`$ notations denote the grid-filter and Favre-filter operators, respectively, while the hat notation will refer to the spatial-filter operator

$$
\begin{aligned}
\widehat{\vec{a}}_{i}(\boldsymbol{x}) & =\int_{\mathscr{D}} G(\boldsymbol{x}-z ; \bar{\Delta}) \breve{a}_{i}(z) \mathrm{d}^{3} z, \\
\int_{\mathscr{D}} G(\boldsymbol{x}-z ; \bar{\Delta}) \mathrm{d}^{3} z & =1, \\
\breve{a}_{i} & =\frac{\left\langle\rho a_{i}\right\rangle}{\langle\rho\rangle} .
\end{aligned}
$$

The function $G(x-z ; \bar{\Delta})$ is the filter kernel, with the characteristic length scale $\bar{\Delta}$. The integration is performed on a compact subset of $\mathbb{R}^{3}$, denoted by $\mathscr{D}$. The usual indicial notation was used, and $\delta_{i j}$ denotes the Kronecker- $\delta$ function, $R e$ is the Reynolds number, $\operatorname{Pr}$ is the Prandtl number (taken to be 0.72), $M_{1}$ is the upstream 
Mach number and $\gamma$ is the specific heat ratio (taken to be 1.4). The reference values to normalise the flow variables are taken in the potential flow, upstream of the interaction. The reference length scale will vary during the discussion and will be explicitly defined where it is used. The subgrid-scale (SGS) stress tensor on the right-hand sides of $(2.1 b)$ and $(2.1 c)$ is

$$
\sigma_{i j}=\left\langle\rho u_{i} u_{j}\right\rangle-\frac{\left\langle\rho u_{i}\right\rangle\left\langle\rho u_{j}\right\rangle}{\langle\rho\rangle},
$$

and the SGS Reynolds heat flux on the right-hand side of $(2.1 c)$ is

$$
\Psi_{j}=\frac{\left\langle\rho T u_{j}\right\rangle}{\langle\rho\rangle}-\frac{\langle\rho T\rangle}{\langle\rho\rangle} \frac{\left\langle\rho u_{j}\right\rangle}{\langle\rho\rangle} .
$$

Note that the right-hand sides of $(2.1 b)$ and $(2.1 c)$ are incomplete. The list of the neglected SGS terms can be found in Touber \& Sandham (2008), together with the motivations which led to the above approximate form of the filtered equations. The SGS stress tensor is modelled via the classical eddy-viscosity approach,

$$
\sigma_{i j}-\frac{1}{3} \delta_{i j} \sigma_{k k}=-2\langle\rho\rangle v_{t} S_{i j}^{\star}
$$

where $v_{t}$ is the eddy viscosity and $S_{i j}^{\star}$ is the deviatoric part of the strain-rate tensor computed from the filtered velocity field:

$$
\begin{aligned}
S_{i j} & =\frac{1}{2}\left(\frac{\partial \breve{u}_{i}}{\partial x_{j}}+\frac{\partial \breve{u}_{j}}{\partial x_{i}}\right), \\
S_{i j}^{\star} & =S_{i j}-\frac{1}{3} \delta_{i j} S_{k k} .
\end{aligned}
$$

The eddy viscosity is then obtained from the mixed-time-scale (MTS) model by Inagaki, Kondoh \& Nagano (2005):

$$
\begin{aligned}
v_{t} & =C_{M} k_{e s} T_{S}, \\
k_{e s} & =\left[\breve{u}_{i}-\widehat{\breve{u}}_{i}\right]\left[\breve{u}_{i}-\widehat{\breve{u}}_{i}\right], \\
T_{S}^{-1} & =\left(\frac{\bar{\Delta}}{\sqrt{k_{e s}}}\right)^{-1}+\left(\frac{C_{T}}{\left|S^{\star}\right|}\right)^{-1},
\end{aligned}
$$

where $\left|S^{\star}\right|^{2}=2 S_{i j}^{\star} S_{i j}^{\star}$ and the constants $C_{M}$ and $C_{T}$ are set to 0.03 and 10 , respectively (see Touber \& Sandham 2009b).

The filter used in the code is a simple top-hat filter with characteristic width equal to the grid spacing. The flow is filtered only in the streamwise and spanwise directions, avoiding issues related to filtering in the stretched-grid direction. The filter size was defined as

$$
\bar{\Delta}=\sqrt{\Delta x \Delta z}
$$

with $\Delta x$ and $\Delta z$ being the grid spacing in the streamwise and spanwise directions, respectively. Once the eddy viscosity is obtained, the SGS heat flux is modelled as

$$
\Psi_{i}=-\frac{v_{t}}{P r_{t}} \frac{\partial \tilde{T}}{\partial x_{i}},
$$

where $v_{t}$ is taken from the SGS stress tensor model. The SGS turbulent Prandtl number $\operatorname{Pr}_{t}$ could, in theory, be computed dynamically as in Moin et al. (1991). However, we consider it to be constant here (as in Garnier, Sagaut \& Deville 2002), with $\operatorname{Pr}_{t}=1.0$. 
The aforementioned governing equations are solved using a fourth-order central spatial differencing scheme for the spatial derivatives and the third-order explicit Runge-Kutta scheme to integrate in time. The boundary treatment is also fourthorder (Carpenter, Nordstrom \& Gottlieb 1998). The code makes use of entropy splitting of the Euler terms and a Laplacian formulation of the viscous terms to enhance the stability of the non-dissipative central scheme (Sandham, Li \& Yee 2002). In addition, a variant of the standard total variation diminishing scheme is used for shock capturing (Yee, Sandham \& Djomehri 1999), coupled with the Ducros sensor (Ducros et al. 1999). Periodic boundary conditions are used in the spanwise direction, while the no-slip condition is enforced at the wall, which is set to be isothermal and equal to the upstream adiabatic wall temperature. The top (freestream) and outflow boundaries make use of an integrated characteristic scheme (Thompson 1987; Sandhu \& Sandham 1994) in order to minimise unwanted reflections from the computational-box boundaries. The oblique shock is introduced at the top boundary using the Rankine-Hugoniot relationships. The code was made parallel in all three directions using MPI libraries.

One major challenge in DNS and LES of wall-bounded turbulence is the need to specify realistic three-dimensional and time-varying inflow conditions. Moreover, in the present SBLI study, it is important to ensure that no artificial low-frequency forcing is introduced in the simulation domain, potentially interfering with the reflected shock dynamics. This was the main motivation to develop a modified version of the digital filter (DF) approach (Klein, Sadiki \& Janicka 2003). The details of the DF approach used in the present simulations can be found in Touber \& Sandham $(2009 \mathrm{~b})$, where it is shown that no particular low-frequency forcing is introduced by this choice of inlet conditions.

\subsection{Flow cases and computational set-ups}

The present work is based on the shock-reflection experiments from the Institut Universitaire des Systèmes Thermiques Industriels (IUSTI) in Marseilles (France). It consists in a Mach 2.3 turbulent boundary layer impinged by an oblique shock generated by a wedge placed in the potential flow. In Dupont et al. (2006), the wedge angle varies from $7^{\circ}$ to $9.5^{\circ}$. In the LES, only the $8^{\circ}$ case will be considered, as it is reported to be unsteady with an interaction length smaller than the $9.5^{\circ}$ case, reducing the computational cost. In this paper, the interaction length $L$ is defined as the distance between the mean reflected-shock-foot position $\bar{x}_{0}$, defined as the linear prolongation of the mean reflected shock to the wall, and the theoretical incidentshock impingement location on the wall in the absence of the boundary layer, $\bar{x}_{i m p}$ (i.e. $L=\bar{x}_{i m p}-\bar{x}_{0}$ ). The separation length is defined as the distance between the mean reattachment location $\bar{x}_{a t}$ and the mean separation location $\bar{x}_{\text {sep }}$ (i.e. $L_{\text {sep }}=\bar{x}_{a t}-\bar{x}_{\text {sep }}$ ). The Reynolds number $R e_{\delta_{2}}$, based on the boundary-layer momentum thickness $\delta_{2}$ (using the classical compressible formulation) upstream of the interaction, is $5 \times 10^{3}$.

The computational settings are detailed in table 1. Two LES simulations will be considered whose results and validations were published in recent papers. The first simulation, labelled narrow-span LES, is discussed in Touber \& Sandham (2009b) whereas the second one, labelled large-span LES, is discussed in Touber \& Sandham $(2009 a)$. The main difference between the two cases is the spanwise extent of the computational domain to allow longer integration times for the low-frequency studies (see table 1). The effect of the spanwise confinement on the interaction was presented in Touber \& Sandham (2009c) and Touber (2010), where it is shown to lead to longer interaction lengths and enhanced low-frequency motions. 


\begin{tabular}{lllll}
\hline & Narrow span & Large span & GSD & P\&G \\
Upstream Reynolds number $R e_{\delta_{2}}$ & $5 \times 10^{3}$ & $5 \times 10^{3}$ & $5 \times 10^{3}$ & $4 \times 10^{3}$ \\
Domain size $L_{x}, L_{y}, L_{z}$ in $L_{\text {sep }}$ & $4.3,0.7,0.1$ & $6.9,1.4,1.6$ & $5.5,2.1,0.5$ & $78,6.8,2.3$ \\
Grid size $N_{x}, N_{y}, N_{z}$ & $451,81,37$ & $451,151,281$ & $255,151,55$ & $2650,111,255$ \\
Grid resolution $\Delta x^{+}, \Delta y_{\text {min }}^{+}, \Delta z^{+}$ & $41,1.6,14$ & $33,1.3,12$ & $50,1,18$ & $15,1,6.5$ \\
Ratio $L_{\text {sep }} / \delta_{0}^{i m p}$ & $O(6)$ & $O(3)$ & $O(3)$ & $O(1)$ \\
SGS model & MTS & MTS & MSM & none (DNS) \\
Dynamic viscosity law & Power & Sutherland & Sutherland & Sutherland \\
Inflow conditions & DF & DF & Recycling & BT \\
Time step $\Delta t \bar{u}_{1} / \delta_{0}^{i m p}$ & $2 \times 10^{-3}$ & $2 \times 10^{-3}$ & $6 \times 10^{-4}$ & $8 \times 10^{-4}$ \\
Number of low-frequency cycles & $O\left(6 \times 10^{1}\right)$ & $O\left(3 \times 10^{1}\right)$ & $O(1)$ & $O(1)$
\end{tabular}

TABLE 1. Computational setups of the IUSTI $8^{\circ}$ case: present calculations and earlier ones. GSD, Garnier et al. (2002); P\&G, Pirozzoli \& Grasso (2006); MSM, mixed-scale model (see Sagaut 2005; Garnier et al. 2002); BT, bypass transition (tripped laminar boundary layer using blowing and suction). Number of low-frequency cycles covered by a sine wave at frequency $f=0.035 \bar{u}_{1} / L_{\text {sep }}$.

In addition to the current LES, table 1 provides a comparison with earlier simulations of the same case, although the DNS of Pirozzoli \& Grasso (2006) was run at a lower Reynolds number. It can be seen that the present simulations span 30-60 longer times than earlier works, so that the reported broadband motions of the reflected shock can be captured (see Touber \& Sandham 2009b). Despite the relatively long signal spanned by the large-span simulation, the convergence of the spectra at $S_{t}<10^{-1}$ is not perfect, where $S_{t} \equiv f L / \bar{u}_{1}$. Thus, some parts of the upcoming analysis will only be performed on the narrow-span LES results. Because the flow statistics of the two LES are published in the aforementioned papers, the next section only covers the shock-motion extraction and resulting conditional averages.

\section{Shock motions and conditional averages}

\subsection{Detection of the shock location}

The shock system is first identified using the dilatation rate. On the basis of a carefully chosen threshold value, the shocks can be detected. While this approach is robust in the potential flow, it becomes less and less reliable as one penetrates into the boundary layer, where the shock is significantly weaker and compressible turbulence structures may match the selected threshold value. Nevertheless, spurious data points can be kept to a minimum. Furthermore, the choice of the dilatation rate was found to produce smoother results than specific sensors such as the one from Ducros et al. (1999), which gave step-like results in the potential flow due to the high level of grid stretching.

Next, the extracted instantaneous shock positions are time-averaged to estimate the streamwise extent, along which the shock extraction can be deemed successful. This choice is manual and rather subjective but it aims at selecting a range of streamwise positions which occur a significant number of times. Therefore, extreme but rare shock positions are not considered. Following this choice, the raw data are then clipped to the selected domain and we are left with the final step, consisting of removing most of the remaining spurious points. The last step is performed automatically, where the decision is based on how far a data point is from the mean value. It was decided to 


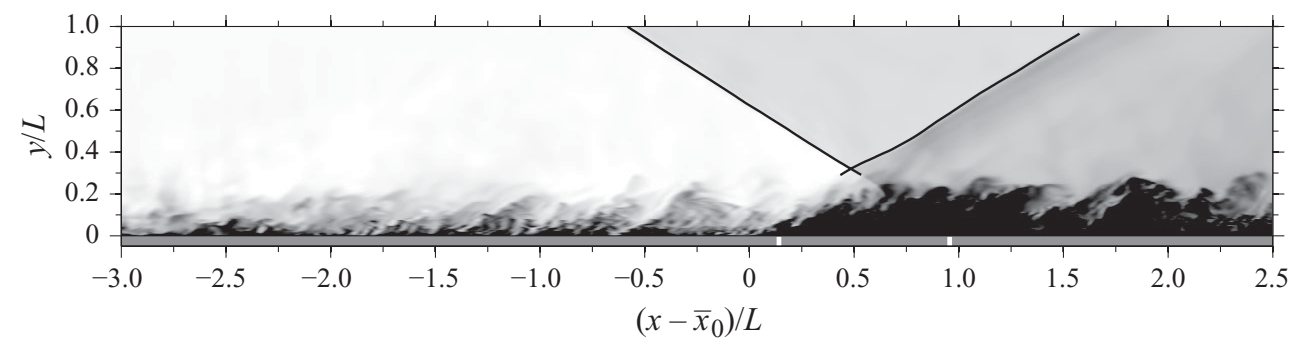

FIGURE 1. Instantaneous side view of the interaction of the large-span simulation with the detected shock system. The grey colour scheme linearly maps the temperature field ranging from hot (black) to cold (white), with $T_{w} / \bar{T}_{1} \approx 2.06$. The black lines indicate the shock system which was captured using the detection algorithm. The thick white ticks incorporated in the wall show the time-averaged separation and reattachment positions.

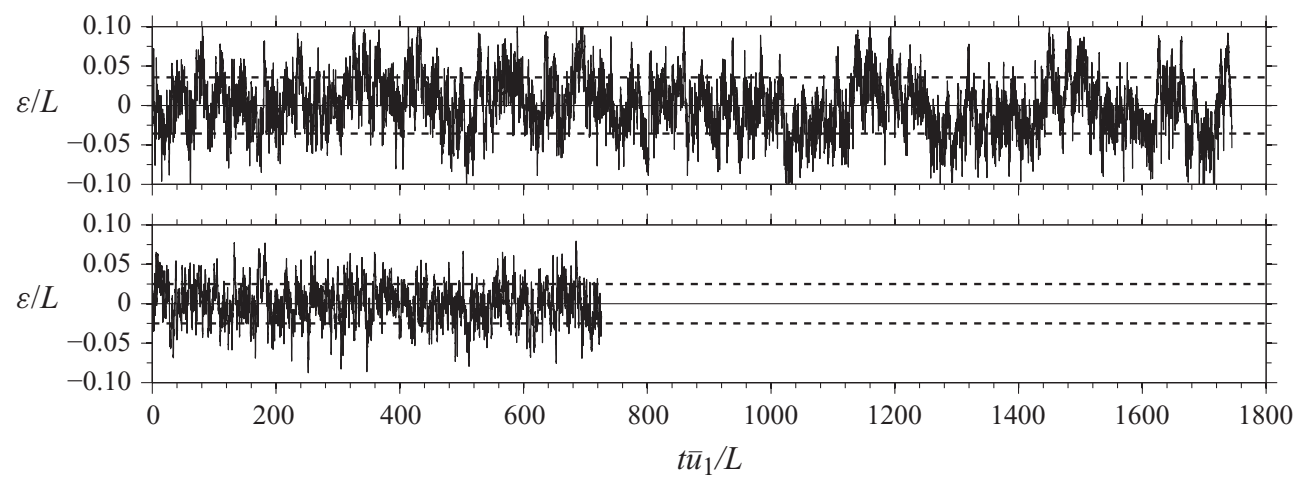

FIGURE 2. Shock-foot-displacement time series from the $(a)$ narrow-span and $(b)$ large-span simulations. The dashed lines indicate the location of the variance $\pm \sigma / L\left(\bar{\varepsilon}=0, \sigma^{2}=\overline{\varepsilon \varepsilon}\right)$.

remove points departing by more than four times the local standard deviation and to replace them using a linear interpolation from the closest instants where the position is reliably known (see Touber 2010 for details).

A snapshot of the end result is provided in figure 1, where one can see the detected shock system and the ability of the method to capture the oscillatory nature of the reflected shock. An animated version of this can be found in Touber \& Sandham (2010).

\subsection{Conditional averages}

The conditional averages used in this work are based on the shock-foot motions extracted from the LES data. By subtracting the time-averaged shock-foot position, the reflected-shock-foot displacement $\varepsilon(t)$ is obtained, as shown in figure 2. The time spanned by the large-span LES is shorter than that covered by the narrow-span LES, due to the computational overhead in the large-span configuration.

Nevertheless, the standard deviation $(\sigma)$ of both raw time series can be computed. The standard deviation is then used as a selection criterion. First, the space spanned by the possible shock-foot positions is split into 12 equally sized bins between $-3 \sigma$ and $+3 \sigma$. Then, for each available instant in the LES database, the flow fields are averaged according to which bin they belong to. 
(a)

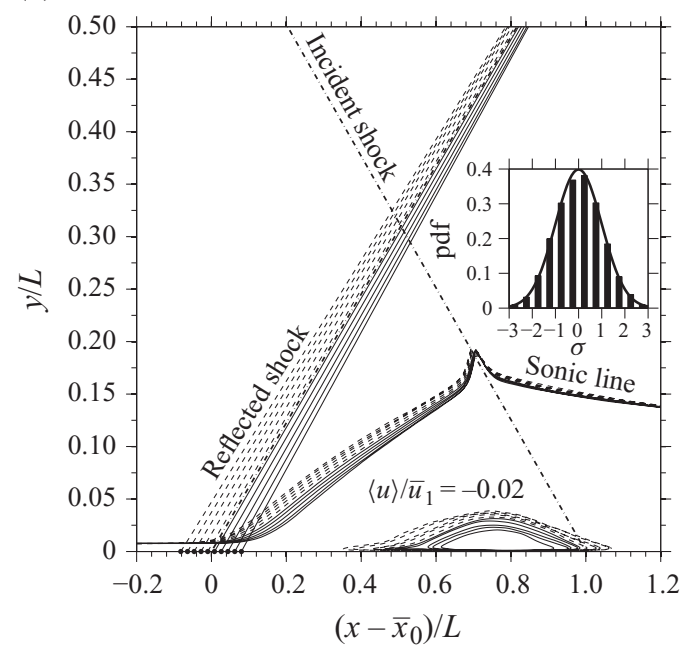

(b)

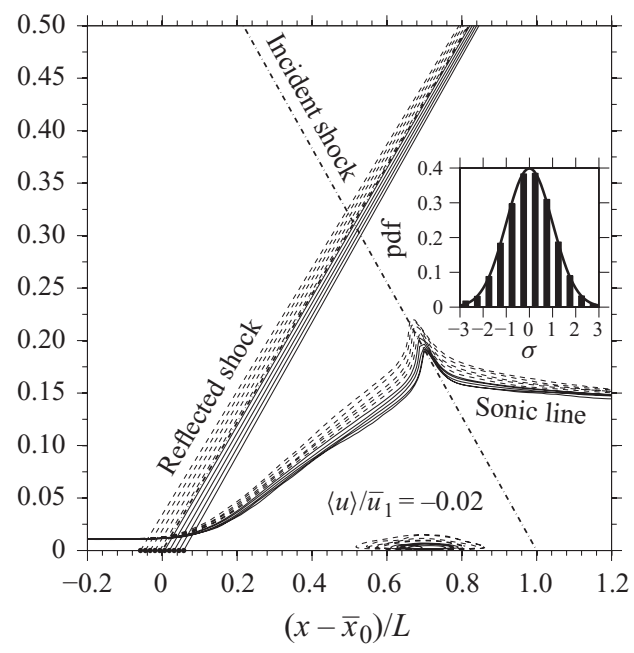

FIGURE 3. Conditional averages of the SBLI based on the probability density function (p.d.f.) of the shock-foot position, which is split into 12 bins in the range $\pm 3 \sigma$. The conditionally averaged shocks are nearly linear but only the best-fit lines are shown in the figure for clarity. Furthermore, the best-fit lines are extended to the wall but in reality the shocks do not penetrate the subsonic region, which is also indicated in the figure. The total number of samples are 160000 and 64990 in the $(a)$ narrow-span and $(b)$ large-span cases, respectively. The normal-law p.d.f. is shown on top of the black histograms.

Let $\mathscr{A}_{\varepsilon_{0}, \Delta \sigma}$ be the set of all the instants $t \in[0, T]$ such that the shock-foot displacement is located between $\varepsilon_{0}$ and $\varepsilon_{0}+\Delta \sigma$, where $T, \varepsilon_{0}$ and $\Delta \sigma$ are some predefined values. Let $\mathscr{N}\left(\mathscr{A}_{\varepsilon_{0}, \Delta \sigma}\right)$ be a measure associated with this set, consisting of the time spanned by $\mathscr{A}_{\varepsilon_{0}, \Delta \sigma}$. This can be written as

$$
\begin{aligned}
\mathscr{A}_{\varepsilon_{0}, \Delta \sigma} & =\left\{t \in[0, T]: \varepsilon(t) \in\left[\varepsilon_{0}, \varepsilon_{0}+\Delta \sigma\right]\right\}, \\
\mathscr{N}\left(\mathscr{A}_{\varepsilon_{0}, \Delta \sigma}\right) & =\int_{\mathscr{A}_{\varepsilon_{0}, \Delta \sigma}} 1 \mathrm{~d} t .
\end{aligned}
$$

The set $\mathscr{A}_{\varepsilon_{0}, \Delta \sigma}$ and its associated measure $\mathscr{N}$ being specified, it is possible to define the conditional-average operator $\langle\cdot\rangle_{\varepsilon_{0}, \Delta \sigma}$ :

$$
\left\langle u_{i}\right\rangle_{\varepsilon_{0}, \Delta \sigma}=\frac{1}{\mathscr{N}\left(\mathscr{A}_{\varepsilon_{0}, \Delta \sigma}\right)} \int_{\mathscr{A}_{\varepsilon_{0}, \Delta \sigma}} u_{i}(t) \mathrm{d} t .
$$

It is straightforward to see that this operator is linear and conserves constants.

Ideally, the LES data could provide $\left\langle u_{i}\right\rangle_{\varepsilon_{0}, \Delta \sigma}$ for any given values of $\varepsilon_{0}$ and $\Delta \sigma$. In practice, this is impossible due to the finite and short time spanned by the LES; and $\varepsilon_{0}, \Delta \sigma$ are chosen such that the range $[-3 \sigma, 3 \sigma]$ can be split into 12 segments. Figure 3 is a plot of the resulting conditionally averaged data. It features the different shock positions (except for the extreme bins), the respective positions of the sonic line as well as the contours where the streamwise velocity is $-0.02 \bar{u}_{1}$. To ease the reading of the figure, the upstream displacements are shown by dashed lines. Note that in the case of figure 3 no effort is made to distinguish the positive $\mathrm{d} \varepsilon / \mathrm{d} t$ events from their negative counterparts. The most interesting aspects of both figures $3(a)$ and $3(b)$ are: (i) the clear correlation between a stronger separation and an upstream 
position of the reflected shock and (ii) the fact that the reflected shock angle does not stay constant between upstream and downstream positions. Note that in both cases, the conditionally averaged shocks are nearly linear and therefore it was decided to approximate them by their best-fit lines. Some differences between the narrowspan and large-span cases may be seen, namely the smaller shock excursions and separation bubbles in the large-span LES and the behaviour of the sonic line, which seems to rotate around a different fixed point. The correlation between the size of the separation and the shock position is a well-established result (see Piponniau et al. 2009). Moreover, the reduction of the streamwise excursions of the shock as one moves further away from the wall was also documented by Dupont et al. (2006). The LES results thus confirm those experimental observations.

From this point onward, we wish to associate the conditional averages with the notion of phase averaging, although we stress that the shock motions are not harmonic so that the notion of phase is different from its usual meaning. At any given position, the velocity-vector time series $u_{i}(t)$ can be decomposed in its time-averaged value $\bar{u}_{i}$ and a time-dependent component $u_{i}^{\prime}(t)$. This is the classical Reynolds decomposition. Now suppose that the time dependency of $u_{i}^{\prime}$ occurs on two distinct time scales, a fast one denoted by $t_{f}$ and a slow one denoted by $t_{s}$ such that $t_{f} / t_{s} \ll 1$. In the present case, $t_{f}$ is associated with the time scales of turbulent structures in the upstream boundary layer whereas $t_{s}$ is associated with the time scales of the low-frequency shock motions. This can be made more formal by setting

$$
\left.\begin{array}{l}
t_{f} \equiv \delta_{0} / \bar{u}_{1}, \\
t_{s} \equiv t_{f} / r_{0}, \text { with } r_{0} \ll 1
\end{array}\right\}
$$

From Dupont et al. (2006) and Touber \& Sandham (2009b), it is known that for the shock-reflection case considered in this work, $r_{0} \sim 10^{-2}$. Thus, the time-dependent component $u_{i}^{\prime}$ is decomposed into the low-frequency $\left(\tilde{u}_{i}\right)$ and high-frequency $\left(u_{i}^{\prime \prime}\right)$ contributions:

$$
u_{i}(t) \equiv \bar{u}_{i}+\tilde{u}_{i}\left(t_{s}\right)+u_{i}^{\prime \prime}\left(t_{f}\right)
$$

By definition, the time average of all fluctuations is zero, i.e. $\overline{u_{i}^{\prime}}=0$. This implies that $\overline{u_{i}^{\prime \prime}}=-\overline{\tilde{u}_{i}}$, which is still too general for the present purposes. Thus, it is also required that each mean contribution vanishes:

$$
\begin{aligned}
\overline{\tilde{u}_{i}} & =0, \\
\overline{u_{i}^{\prime \prime}} & =0 .
\end{aligned}
$$

At this stage, it is tempting to try to relate $\tilde{u}_{i}$ with the conditionally averaged fields $\left\langle u_{i}\right\rangle_{\varepsilon_{0}, \Delta \sigma}$, but this is not trivial. The main difficulty in reconciling the two resides in the temporal dependence of $\tilde{u}_{i}$, as opposed to the dependence of $\left\langle u_{i}\right\rangle_{\varepsilon_{0}, \Delta \sigma}$ on the selected shock-foot position. To remove this difficulty, the following (strong) hypothesis will be used.

HYPOTHESIS 1. For a given reflected-shock-foot position taken from a low-pass filtered signal (with cutoff frequency $\left.O\left(0.1 \bar{u}_{1} / \delta_{0}\right)\right)$, the associated flow field $\tilde{u}_{i}$ is uniquely defined:

$$
\tilde{u}_{i}\left(t_{s}\right)=\tilde{u}_{i}\left(\varepsilon\left(t_{s}\right)\right) .
$$

The validity of the above hypothesis is debatable but it may be justified in the light of the LES results. While it is clear that there exist an infinite number of different 
flow fields $u_{i}$ yielding the same shock-foot position $x_{0}$ (when for example considering the transverse waves along the shock and the turbulence), it is argued that when only the low-frequency motions are retained, the picture may become uniquely defined. One supporting observation is that the conditionally averaged LES data, where the distinction between upstream and downstream shock motion was made, do not show any significant level of hysteresis. In other words, for a given shock position, the fact that the shock foot was moving upstream or downstream does not matter, giving one example where hypothesis 1 is satisfied. The above arguments strongly depend on the observed scale separation between the low-frequency shock motions and the turbulence-related fluctuations.

If hypothesis 1 is satisfied, and assuming that the turbulence fluctuations do not correlate with the shock-foot motions, making the conditional-averaging operation similar to a time integration (i.e. $\left\langle u_{i}^{\prime \prime}\right\rangle_{\varepsilon_{0}, \Delta \sigma}=\overline{u_{i}^{\prime \prime}}=0$ ), the following two corollaries may be written (the details of which are provided in Appendix A).

Corollary 1.

$$
\left\langle u_{i}\right\rangle_{\varepsilon_{0}, \Delta \sigma}-\bar{u}_{i}=\tilde{u}_{i}\left(\varepsilon_{0}\right)+O(\Delta \sigma)
$$

\section{Corollary 2.}

$$
\int_{\mathscr{A}_{\varepsilon_{0}, \Delta \sigma}} \tilde{u}_{i}\left(\varepsilon\left(t_{s}\right)\right) \tilde{u}_{j}\left(\varepsilon\left(t_{s}\right)\right) \mathrm{d} t_{s}=\left[\tilde{u}_{i}\left(\varepsilon_{0}\right) \tilde{u}_{j}\left(\varepsilon_{0}\right)+O(\Delta \sigma)\right] \mathscr{N}\left(\mathscr{A}_{\varepsilon_{0}, \Delta \sigma}\right) .
$$

As mentioned earlier, the term 'phase average' will be used here to refer to $\tilde{u}_{i}\left(t_{s}\right)$. To quantify the spatial and energetic relevance of the phase fluctuations, it is of interest to compute the phase-fluctuation stress tensor $\overline{\tilde{u}_{i} \tilde{u}_{j}}$. By invoking the previous corollaries, one can easily show that the phase-fluctuation stress tensor may be evaluated from the following sum (see Appendix A):

$$
\overline{\tilde{u}_{i} \tilde{u}_{j}} \approx \frac{1}{T} \sum_{k=0}^{N-1}\left[\left\langle u_{i}\right\rangle_{\varepsilon_{\min }+k \Delta \sigma, \Delta \sigma}-\bar{u}_{i}\right]\left[\left\langle u_{j}\right\rangle_{\varepsilon_{\min }+k \Delta \sigma, \Delta \sigma}-\bar{u}_{j}\right] \mathscr{N}\left(\mathscr{A}_{\varepsilon_{\min }+k \Delta \sigma, \Delta \sigma}\right),
$$

where $N=\left(\varepsilon_{\max }-\varepsilon_{\min }\right) / \Delta \sigma$ with $\varepsilon_{\max }=\max (\varepsilon(t)), \varepsilon_{\min }=\min (\varepsilon(t)), t \in[0, T]$.

Figure 4 compares the distribution of the kinetic energy associated with the phase fluctuations alone with the kinetic energy associated with all fluctuations. In the narrow-span case, the contribution of the phase fluctuations to the total energy represents about $30 \%$ whereas in the large-span case, where the low-frequency motions were found to be less energetic (see Touber \& Sandham 2009c), the contribution of the phase fluctuation is less than $10 \%$. In both cases, the contribution of the phase fluctuations is restricted around the mean reflected-shock position and in the vicinity of the first section of the mixing layer, as one would expect.

\section{Stochastic dynamical model derivation}

\subsection{Initial form of the momentum integral equation}

To derive the model, the streamwise component of the unsteady momentum equation is first integrated in the wall-normal direction. After some algebraic manipulations (provided in Appendix B), one can obtain the following exact form of the momentum 
(a)

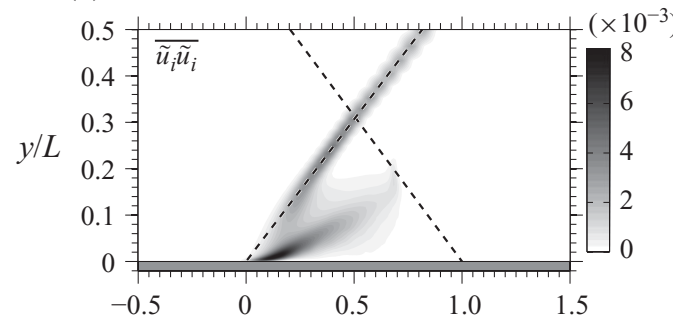

(c)

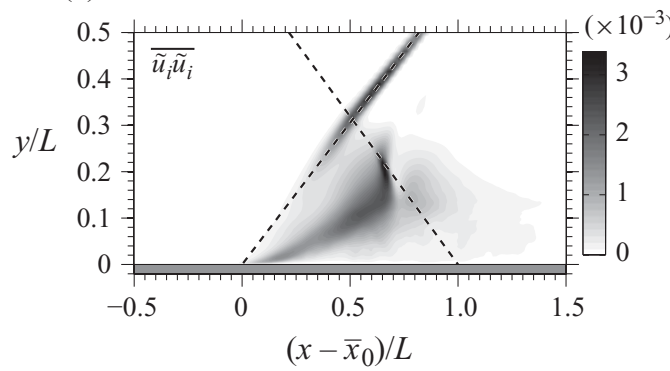

(b)

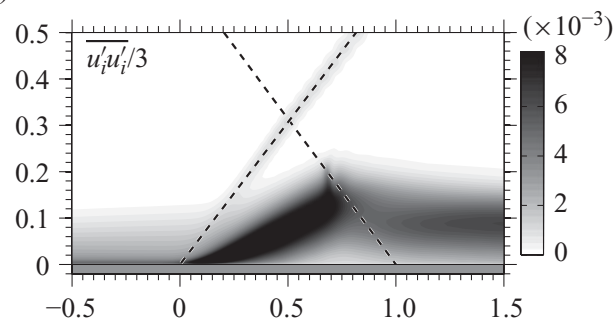

(d)

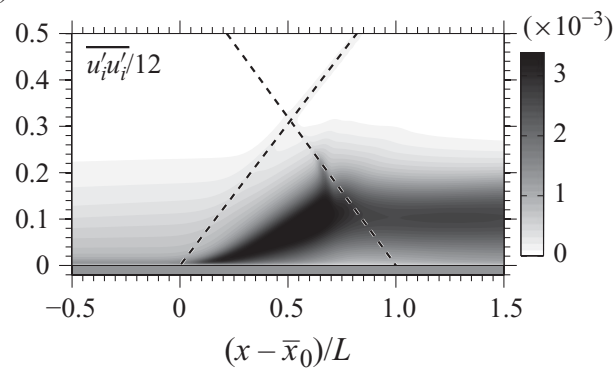

FIGURE 4. Kinetic energy fields from all fluctuations $(b, d)$ and from the phase fluctuations only $(a, c) .(a, b)$ Narrow-span case (note the factor $1 / 3$ in $(b)) ;(c, d)$ large-span case (note the factor $1 / 12$ in $(d))$. The dashed lines indicate the position of the shocks.

integral equation (MIE):

$$
\begin{gathered}
\rho_{h}\left(h-\delta_{1}\right)\left[\frac{\partial u_{h}}{\partial t}+u_{h} \frac{\partial u_{h}}{\partial x}\right]+u_{h} \frac{\partial}{\partial t}\left[\rho_{h}\left(\delta_{\rho}-\delta_{1}\right)\right]-\frac{\partial}{\partial x}\left[\rho_{h} u_{h}^{2} \delta_{2}\right]+\frac{\partial}{\partial z}\left[\int_{0}^{h} \rho u w \mathrm{~d} y\right] \\
-u_{h} \frac{\partial}{\partial z}\left[\int_{0}^{h} \rho w \mathrm{~d} y\right]=\frac{\partial}{\partial x}\left[p_{h}\left(\delta_{p}-h\right)\right]+\frac{\mu_{h}}{R e}\left[\left.\frac{\partial v}{\partial x}\right|_{y=h}+\left.\frac{\partial u}{\partial y}\right|_{y=h}\right]-\frac{1}{2} C_{f} \rho_{h} u_{h}^{2} \\
+\frac{1}{R e} \frac{\partial}{\partial x}\left[\int_{0}^{h} \tau_{x x} \mathrm{~d} y\right]+\frac{1}{R e}\left(\frac{\partial}{\partial z}\left[\int_{0}^{h} \tau_{x z} \mathrm{~d} y\right]-\left.\tau_{x z}\right|_{y=h} \frac{\partial h}{\partial z}\right)
\end{gathered}
$$

where the following thicknesses are defined (displacement, momentum, pressure and density thicknesses, respectively):

$$
\begin{aligned}
\delta_{1} & =\int_{0}^{h}\left(1-\frac{\rho u}{\rho_{h} u_{h}}\right) \mathrm{d} y \\
\delta_{2} & =\int_{0}^{h} \frac{\rho u}{\rho_{h} u_{h}}\left(1-\frac{u}{u_{h}}\right) \mathrm{d} y, \\
\delta_{p} & =\int_{0}^{h}\left(1-\frac{p}{p_{h}}\right) \mathrm{d} y \\
\delta_{\rho} & =\int_{0}^{h}\left(1-\frac{\rho}{\rho_{h}}\right) \mathrm{d} y
\end{aligned}
$$

and

$$
C_{f}=\left.\frac{2 \mu_{w}}{\rho_{h} u_{h}^{2} R e} \frac{\partial u}{\partial y}\right|_{w}
$$




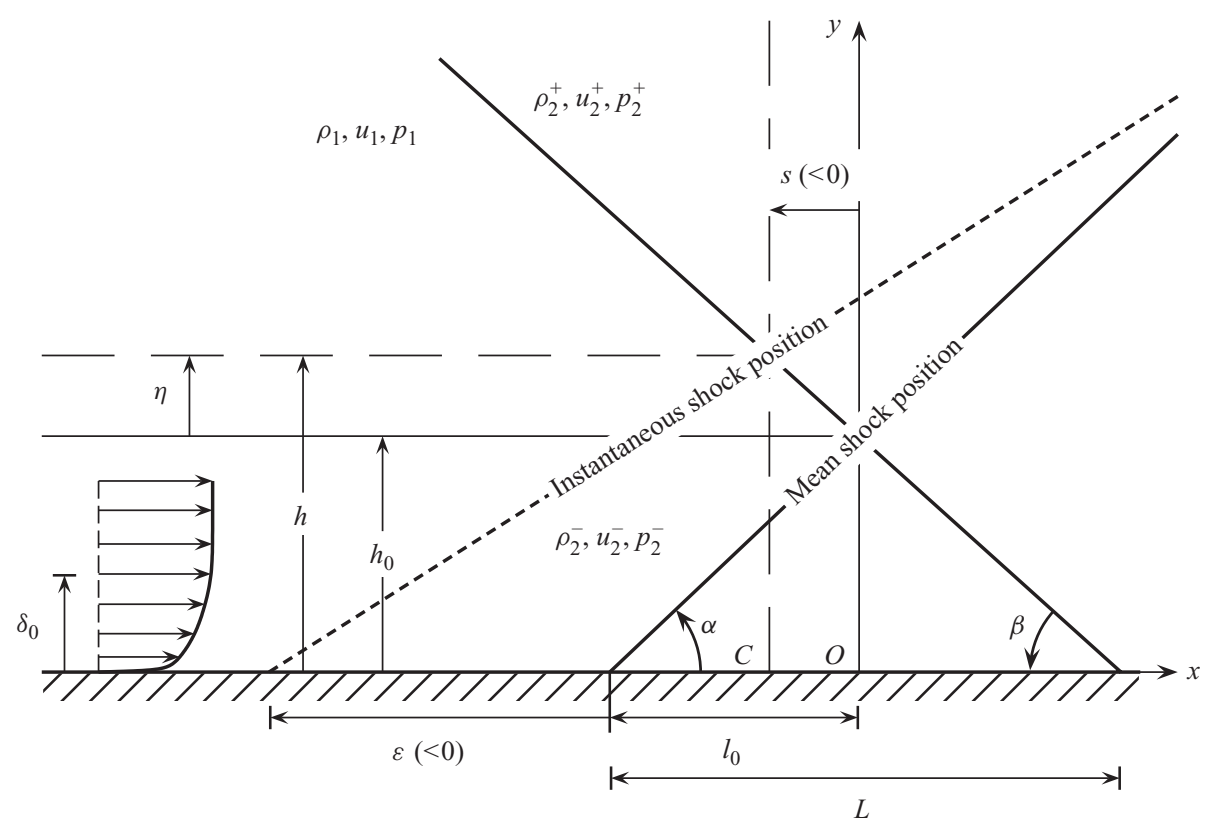

FIGURE 5. Sketch of the interaction with the definition of the notations in use.

where the subscripts $h$ and $w$ denote that variables are evaluated at $y=h$ and at the wall, respectively, with $h$ being the distance measured from the wall of the instantaneous shock-crossing point, as shown in figure 5.

\subsection{Change of variable}

It is convenient to introduce a new coordinate system by moving the origin of the streamwise $x$ axis to the instantaneous shock-foot position and then normalising with the distance covered from the instantaneous shock foot to the instantaneous shockcrossing point (denoted by $C$ in figure 5 ). Note that $l_{0}$ is the absolute distance between the mean shock-foot position and the mean streamwise cross-point position whereas $x$, $s$ and $\varepsilon$ can be either positive or negative distances, with $s$ and $\varepsilon$ respectively denoting the instantaneous shock-crossing and shock-foot positions relative to the mean (see figure 5). With the upstream movement of the shock foot sketched in figure 5, $s$ and $\varepsilon$ are negative. The distance from the origin of the axis system $O$ to the instantaneous shock-foot location is $l_{0}-\varepsilon$ and the distance separating the instantaneous shock foot from the instantaneous crossing point is $l_{0}-\varepsilon+s$. Therefore, the new coordinate system, denoted by $\xi$, is

$$
\xi \equiv \frac{x+l_{0}-\varepsilon}{l_{0}-\varepsilon+s} \text { or equivalently, } x \equiv\left(l_{0}-\varepsilon\right)(\xi-1)+s \xi .
$$

Hence, in the following sections, $\xi=0$ is the instantaneous shock-foot position, $\varepsilon$ is the shock-foot displacement with respect to its mean position and $\xi=1$ is the instantaneous location of the shock crossing.

Because of the integration in the wall-normal directions, the terms in (4.1) are only functions of $x, z$ and $t$. This can be expressed in a generic way by writing that the terms in (4.1) are of the type $f(x, z, t)$. Equation (4.4) will transform $f(x, z, t)$ into $f(\xi(z, t))$. From the chain rule, $\partial f / \partial(\cdot)=[\partial f / \partial \xi][\partial \xi / \partial(\cdot)]$, where $(\cdot)$ should be replaced by $t, x$ and $z$. From (4.4), it is straightforward to compute the following 
derivatives:

$$
\begin{aligned}
\frac{\partial \xi}{\partial t} & =\frac{1}{l_{0}-\varepsilon+s}\left[(\xi-1) \frac{\partial \varepsilon}{\partial t}-\xi \frac{\partial s}{\partial t}\right], \\
\frac{\partial \xi}{\partial x} & =\frac{1}{l_{0}-\varepsilon+s}, \\
\frac{\partial \xi}{\partial z} & =\frac{1}{l_{0}-\varepsilon+s}\left[(\xi-1) \frac{\partial \varepsilon}{\partial z}-\xi \frac{\partial s}{\partial z}\right] .
\end{aligned}
$$

Using the above relations, one can express (4.1) in the new coordinate system:

$$
\begin{aligned}
& \frac{\rho_{h}\left(h-\delta_{1}\right)}{l_{0}-\varepsilon+s}\left[(\xi-1) \frac{\partial \varepsilon}{\partial t}-\xi \frac{\partial s}{\partial t}+u_{h}\right] \frac{\partial u_{h}}{\partial \xi} \\
& +\frac{1}{l_{0}-\varepsilon+s}\left[u_{h}\left((\xi-1) \frac{\partial \varepsilon}{\partial t}-\xi \frac{\partial s}{\partial t}\right) \frac{\partial}{\partial \xi}\left[\rho_{h}\left(\delta_{\rho}-\delta_{1}\right)\right]-\frac{\partial}{\partial \xi}\left[\rho_{h} u_{h}^{2} \delta_{2}\right]\right] \\
& \quad+\frac{1}{l_{0}-\varepsilon+s}\left[(\xi-1) \frac{\partial \varepsilon}{\partial z}-\xi \frac{\partial s}{\partial z}\right]\left\{\frac{\partial}{\partial \xi}\left[\int_{0}^{h} \rho u w \mathrm{~d} y\right]-u_{h} \frac{\partial}{\partial \xi}\left[\int_{0}^{h} \rho w \mathrm{~d} y\right]\right\} \\
& =\frac{1}{l_{0}-\varepsilon+s} \frac{\partial}{\partial \xi}\left[p_{h}\left(\delta_{p}-h\right)\right]+\frac{\mu_{h}}{R e}\left[\left.\frac{1}{l_{0}-\varepsilon+s} \frac{\partial v}{\partial \xi}\right|_{y=h}+\left.\frac{\partial u}{\partial y}\right|_{y=h}\right]-\frac{1}{2} C_{f} \rho_{h} u_{h}^{2} \\
& \quad+\frac{1}{R e} \frac{1}{l_{0}-\varepsilon+s} \frac{\partial}{\partial \xi}\left[\int_{0}^{h} \tau_{x x} \mathrm{~d} y\right] \\
& \quad+\frac{1}{R e} \frac{1}{l_{0}-\varepsilon+s}\left[(\xi-1) \frac{\partial \varepsilon}{\partial z}-\xi \frac{\partial s}{\partial z}\right]\left\{\frac{\partial}{\partial \xi}\left[\int_{0}^{h} \tau_{x z} \mathrm{~d} y\right]-\left.\tau_{x z}\right|_{y=h} \frac{\partial h}{\partial \xi}\right\} .
\end{aligned}
$$

\subsection{Approximate form of the momentum integral equation}

In principle, if one could find all the appropriate necessary closure terms, (4.6) would be used to resolve the shock dynamics. However, in its current state, (4.6) is unpractical and one needs to make further assumptions in order to simply it. Some reasonable assumptions are:

(a) the study shall be restricted to $\xi<1$;

(b) the potential flow is assumed constant (e.g. the acoustic field is neglected) so that $u_{1}, \rho_{1}$ and $p_{1}$ are true constants $\left(\rho_{1}=\bar{\rho}_{1}, u_{1}=\bar{u}_{1}, p_{1}=\bar{p}_{1}\right)$;

(c) the top boundary (delimited by $h$ in figure 5) is assumed to be always inside the potential flow, i.e. $h>\delta_{0}$ at all times;

(d) the shock system is considered two-dimensional (i.e. spanwise variations are not considered), so that $h=h(t), s=s(t), \varepsilon=\varepsilon(t)$ (three-dimensional effects could be considered in a future study).

With the above assumptions, the subscripts $h$ can be replaced by 1 (e.g. $u_{h}=u_{1}$ ), since $h$ is inside the potential flow (assumption $c$ ) and the study restricted to the section upstream of the shock-crossing point (assumption $a$ ). Furthermore, for a constant potential flow (assumption $b$ ), one can write $\partial u_{h} / \partial \xi=\partial u_{1} / \partial \xi=0$. For similar reasons, multiplicative terms such as $\rho_{h}, u_{h}$ or $p_{h}$ can be pulled out of derivatives. The two-dimensional (2D) assumption (assumption $d$ ) is used to zero out terms with $\partial / \partial z$. Finally, the shear-stress term $\left.\tau_{x z}\right|_{y=h}$ vanishes under assumptions $b$ and $c$. Implementing the above simplifications to (4.6) eventually leads to the 
(a) Magnitude of the MIE terms

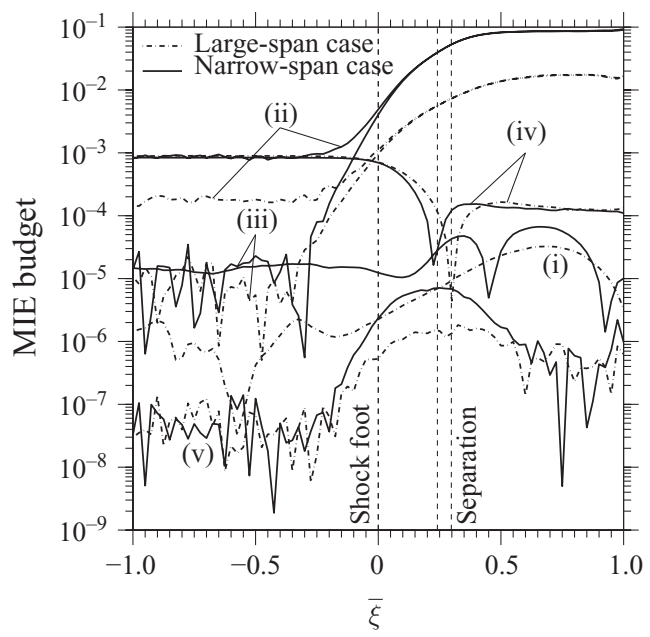

(b) Variance of the MIE terms

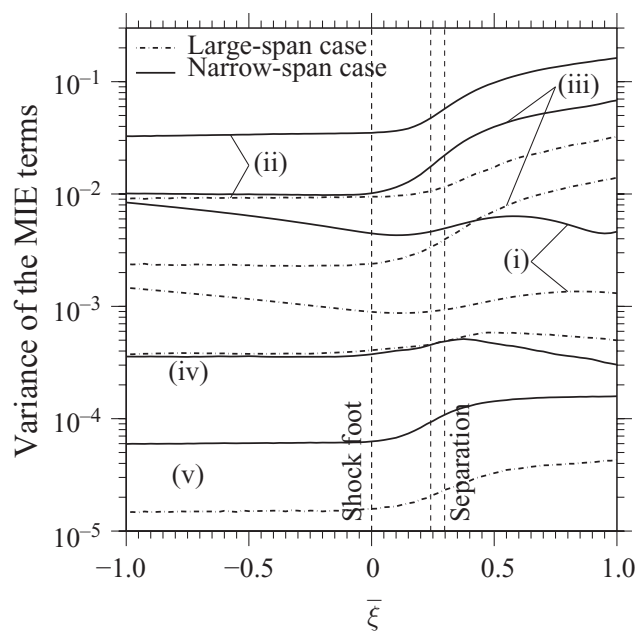

FiguRE 6. Evaluation of the contribution from each term in (4.7) to the $(a)$ time-averaged balance and $(b)$ their relative importance to the energy of the fluctuations. Note that most of the data smaller than $O\left(10^{-5}\right)$ are subject to noise.

following approximate form of the MIE:

$$
\begin{aligned}
\underbrace{\frac{1}{u_{1} l_{0}}\left[(1-\xi) \frac{\mathrm{d} \varepsilon}{\mathrm{d} t}+\xi \frac{\mathrm{d} s}{\mathrm{~d} t}\right] \frac{\partial}{\partial \xi}\left[\delta_{\rho}-\delta_{1}\right]}_{(\mathrm{i})}+\underbrace{\frac{1}{l_{0}} \frac{\partial \delta_{2}}{\partial \xi}}_{(\mathrm{ii})}+\underbrace{\frac{p_{1}}{\rho_{1} u_{1}^{2} l_{0}} \frac{\partial \delta_{p}}{\partial \xi}}_{(\mathrm{iii})} \\
=\underbrace{\frac{1}{2}\left(1-\frac{\varepsilon}{l_{0}}+\frac{s}{l_{0}}\right) C_{f}}-\underbrace{\frac{1}{\rho_{1} u_{1}^{2} l_{0} \operatorname{Re}} \frac{\partial}{\partial \xi}\left[\int_{0}^{h} \tau_{x x} \mathrm{~d} y\right]} .
\end{aligned}
$$

In a canonical boundary layer, term (v) would be neglected and it is worth checking if this would also hold for the current SBLI configuration. Each term in (4.7) is therefore evaluated using the LES data and the magnitudes are shown in figure 6(a). It can be seen that upstream of the interaction, (v) is $O\left(10^{-7}\right)$ whereas all the other terms are greater than $O\left(10^{-5}\right)$, justifying the common assumption made in canonical boundary layers. Upon entering the interaction region, the amplitude of (v) rises, as one would expect, to reach a maximum (for the region considered here) near separation. However, it may be argued that this maximum remains small compared with the other terms, with the exception of the skin-friction term (iv) right at separation, where it is strictly zero. Because the analysis of the time-averaged data is not sufficient to judge the relevance of $(\mathrm{v})$ in the unsteady context, the relative importance of the variance of each terms in (4.7) is also considered in figure $6(b)$. It is found that term (v) only makes a marginal contribution to the energy of the fluctuations and it appears justified, as a leading-order approximation, to neglect (v) from (4.7).

In figure $6(a)$, it is also interesting to note that on average within the interaction, there is an approximate balance between the rate of changes of momentum and pressure thicknesses (terms (ii) and (iii)). At leading order, those two terms control the interaction length by setting the necessary equilibrium between the adverse pressure gradient and the rate of change of momentum thickness. We shall come back to this point later. 

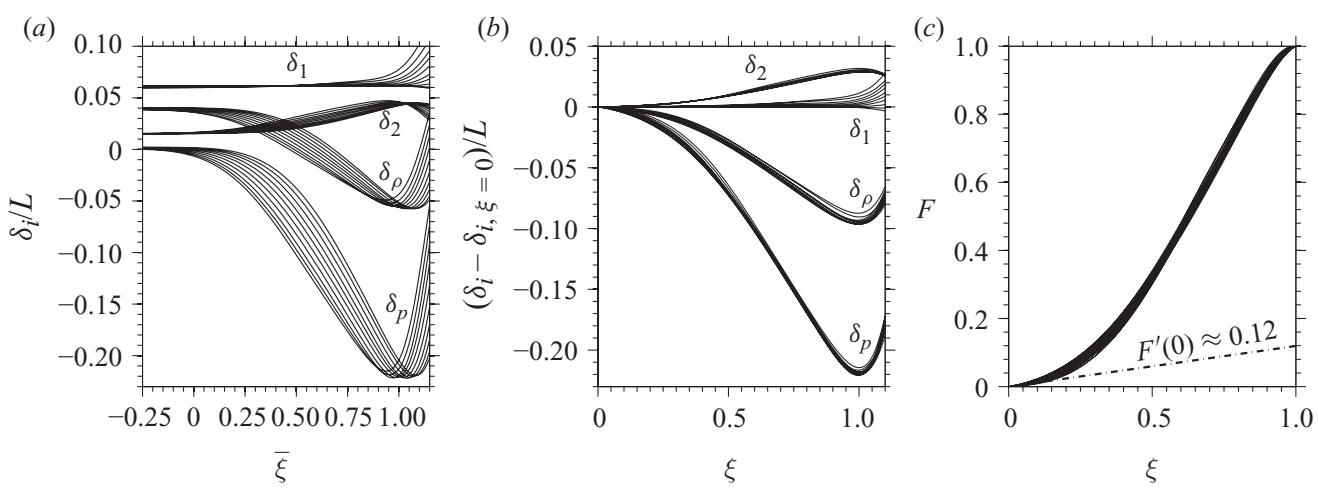

FIGURE 7. $(a-c)$ Validity of hypothesis 2 from the conditionally averaged LES data; see text for details.

\subsection{Hypothesis of the existence of a similarity solution}

Equation (4.7) with (v) neglected is not yet in a closed form but some interesting features are already emerging. The final dynamical equation which is sought is the governing equation for $\varepsilon$ and, looking at (4.7), some terms in $\varepsilon$ can already be identified amongst terms involving the streamwise evolutions of the various thicknesses. One common approach to transforming a partial differential equation into an ordinary one is to seek similarity solutions. In this particular case, one can attempt to map terms in $\partial / \partial \xi$ into a family of functions playing the role of coefficients in the final governing equation for the shock motions. Such families can indeed arise if the following hypothesis is invoked.

Hypothesis 2. There exists a similarity function $(F)$ that describes the streamwise evolution of the various boundary-layer thicknesses independent of the time variable, i.e.

$$
\left.\begin{array}{l}
F(\xi) \equiv \frac{\delta_{i}(\xi)-\delta_{i}(\xi=0)}{\Delta_{i}}, \\
\Delta_{i}(t) \equiv \delta_{i}(\xi=1)-\delta_{i}(\xi=0),
\end{array}\right\}
$$

where the subscript $i$ is any of the following: 1, 2, $\rho, p$.

Mathematically, hypothesis 2 corresponds to the supposed existence of a separation of variables. From (4.8),

$$
\delta_{i}(\xi)=F(\xi) \Delta_{i}(t)+\delta_{i}(\xi=0), \frac{\partial \delta_{i}}{\partial \xi}=\frac{\mathrm{d} F}{\mathrm{~d} \xi} \Delta_{i} \equiv F^{\prime} \Delta_{i},
$$

so that the MIE becomes

$$
\begin{aligned}
\frac{1}{u_{1} l_{0}}\left[(1-\xi) \frac{\mathrm{d} \varepsilon}{\mathrm{d} t}+\xi \frac{\mathrm{d} s}{\mathrm{~d} t}\right]\left(F^{\prime} \Delta_{\rho}-F^{\prime} \Delta_{1}\right)+\frac{1}{l_{0}} F^{\prime} \Delta_{2} & +\frac{p_{1}}{\rho_{1} u_{1}^{2} l_{0}} F^{\prime} \Delta_{p} \\
& =\frac{1}{2}\left(1-\frac{\varepsilon}{l_{0}}+\frac{s}{l_{0}}\right) C_{f} .
\end{aligned}
$$

The validity of hypothesis 2 can be tested using conditionally averaged LES data, as shown in figure 7. In figure 7(a), the $\delta_{i}$ functions are shown from the stationary axis $\bar{\xi}$. The same functions are then plotted in the moving coordinate system $\xi$ (see figure $7 b$ ), making the local extrema in the $\delta_{i}$ distributions centred at $\xi=1$. Finally, the $\delta_{i}$ functions are shifted by $\delta_{i}(\xi=0)$ and normalised by their respective amplitudes $\Delta_{i}$ to give the $F$ functions shown in figure $7(c)$. It is argued that the 30 curves shown in figure $7(c)$ collapse reasonably well onto the hypothesised universal function $F$. 
(a)

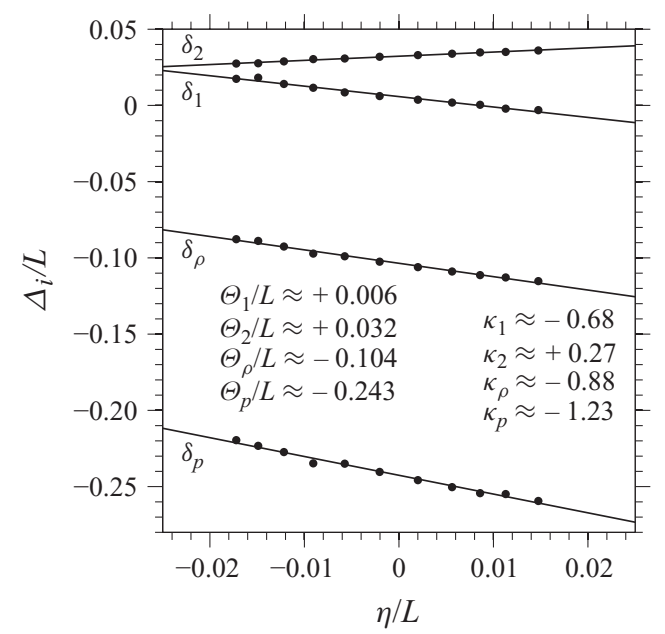

(b)

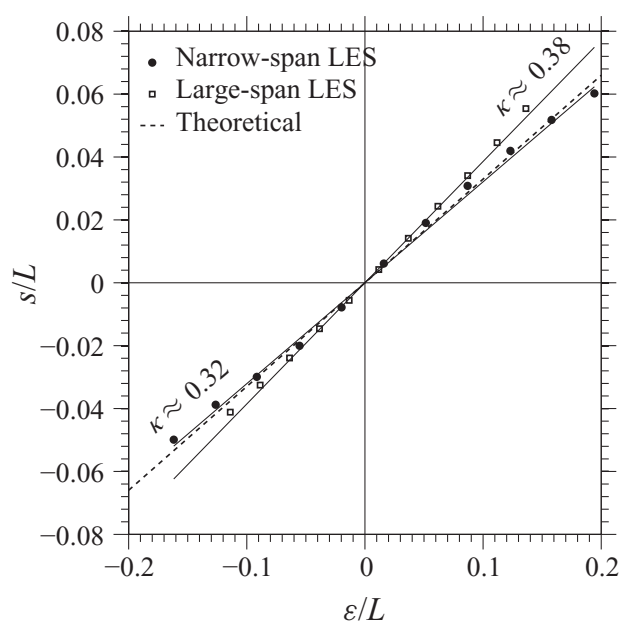

FiguRE 8. $(a)$ Dependency of thicknesses amplification $\left(\Delta_{i}\right)$ on the shock-system position $(\eta)$ and $(b)$ the relationship between the shock-foot motions and the shock-cross point motions. The theoretical line in $(b)$ will be described in $\S 5$.

However, evaluating the thicknesses from the LES fields, and in particular at the shock-crossing point streamwise station $\xi=1$, is difficult due to the shock-smearing and grid-stretching effects, reducing the accuracy of these quantities at this particular station. The plots in figure 7 should thus be regarded as indicative only. Furthermore, one can see in figure 7 that the time-averaged displacement thickness does not increase much between $\xi=0$ and $\xi=1$, compared with the other thicknesses, making the division by $\Delta_{1}$ in (4.8) sensitive to numerical errors. Therefore, the case of the $\delta_{1}$ distributions was not included in figure $7(c)$.

Despite the issues outlined above, the LES data provide good support for hypothesis 2. Looking at (4.10), one also needs to consider the $\Delta_{i}$ functions and these quantities are also difficult to obtain numerically. Nevertheless, figure $8(a)$ gives an idea of how the $\Delta_{i}$ functions depend on the shock-system position. The numerical results suggest that, as a first approximation, the overall changes of the different thicknesses considered here may be approximated by the mean value plus a linear dependence on $\eta$, defined as the shock-crossing point wall-normal displacement (see figure 5):

$$
\Delta_{i}=\Theta_{i}+\kappa_{i} \eta(t) .
$$

The above approximation will be further discussed in $\S 5$. Next, it is easily seen from geometrical considerations (see figure 5) that

$$
\eta(t)=-s(t) \tan \beta
$$

From (4.10) and (4.12), it is clear that a relationship between the shock-foot displacement $\varepsilon$ and the shock-crossing-point streamwise displacement $s$ is needed. This relation is reported in figure $8(b)$ using the LES data sets. Again, a linear relation seems appropriate and reflects the earlier impressions on the conditionally averaged data in figure 3 :

$$
s(t)=k \varepsilon(t) .
$$

The rationale behind (4.13) will be further discussed in $\S 5$ and the theoretical line in figure $8(b)$ will be explained. 
Using (4.11)-(4.13) in (4.10), one can write

$$
\begin{aligned}
& \frac{1}{u_{1}} k \tan \beta\left(\kappa_{1}-\kappa_{\rho}\right) F^{\prime}(\xi)[1+\xi(k-1)] \frac{\mathrm{d} \varepsilon}{\mathrm{d} t} \varepsilon+\frac{L}{u_{1}}\left(\frac{\Theta_{\rho}}{L}-\frac{\Theta_{1}}{L}\right) F^{\prime}(\xi)[1+\xi(k-1)] \frac{\mathrm{d} \varepsilon}{\mathrm{d} t} \\
& \quad+\left[\frac{1}{2} C_{f}(1-k)-k \tan \beta\left(\kappa_{2}+\frac{p_{1}}{\rho_{1} u_{1}^{2}} \kappa_{p}\right) F^{\prime}(\xi)\right] \varepsilon \\
& =L\left(\frac{1}{2} \frac{l_{0}}{L} C_{f}-\frac{\Theta_{2}}{L} F^{\prime}(\xi)-\frac{p_{1}}{\rho_{1} u_{1}^{2}} \frac{\Theta_{p}}{L} F^{\prime}(\xi)\right) .
\end{aligned}
$$

Since the location of interest in this study is the shock foot, the parameter $\xi$ can be set to zero. Allowing for $F^{\prime}(0)$ being non-zero (figure 7) and introducing the following non-dimensional variables,

$$
\left.\begin{array}{l}
\zeta \equiv \varepsilon / L, \\
t^{\star} \equiv t u_{1} / L, \\
\dot{\zeta} \equiv \mathrm{d} \zeta / \mathrm{d} t^{\star}
\end{array}\right\}
$$

(4.14) becomes

$$
\begin{array}{r}
k \tan \beta\left(\kappa_{1}-\kappa_{\rho}\right) \zeta \dot{\zeta}+\left(\frac{\Theta_{\rho}}{L}-\frac{\Theta_{1}}{L}\right) \dot{\zeta}+\left[\frac{C_{f}(0)}{2 F^{\prime}(0)}\right. \\
\left.(1-k)-k \tan \beta\left(\kappa_{2}+\frac{p_{1}}{\rho_{1} u_{1}^{2}} \kappa_{p}\right)\right] \zeta \\
=\frac{l_{0} C_{f}(0)}{2 L F^{\prime}(0)}-\frac{\Theta_{2}}{L}-\frac{p_{1}}{\rho_{1} u_{1}^{2}} \frac{\Theta_{p}}{L} .
\end{array}
$$

Equation (4.16) is a first-order nonlinear ODE representing the shock-foot motions in the presence of the forcing term $C_{f}(\xi=0)$ (note that separation occurs for $\xi>0$ so that $\left.C_{f}(\xi=0)>0\right)$. For particular cases, the constants could be computed from the LES, but for more general applications we need to model them. Prior to discussing some tentative modelling efforts, it is of interest to use the LES data to perform a leading-order analysis and further simplify the governing equation (4.16).

\subsection{Leading-order equations}

To further simplify the equation for the shock-foot motions, it is convenient to apply the triple decomposition approach introduced earlier (see $(3.3 a)$ ) to decompose the skin-friction time series at the shock foot:

$$
C_{f}(\xi=0)=\bar{C}_{f_{0}}+\tilde{C}_{f_{0}}\left(t_{s}\right)+C_{f_{0}}^{\prime \prime}\left(t_{f}\right) .
$$

Terms $\tilde{C}_{f_{0}}$ and $C_{f_{0}}^{\prime \prime}$ correspond to the skin-friction fluctuations at the shock foot associated with the low-frequency motions and the high-frequency fluctuations due to the turbulence, respectively. From the LES time series, it is found that both $\tilde{C}_{f_{0}}$ and $C_{f_{0}}^{\prime \prime}$ contribute to the skin-friction fluctuations and therefore one cannot neglect $C_{f_{0}}^{\prime \prime}$ in (4.17). Furthermore, using the LES conditional averages and invoking the first corollary $(3.5 a)$, it is possible to evaluate the correlation between $\tilde{C}_{f_{0}}$ and the shock displacement $\zeta$, as shown in figure 9. Although there is some departure from the linear relationship at larger positive $\varepsilon$ due to the asymmetry in $\left|\mathrm{d} \bar{C}_{f} / \mathrm{d} x\right|$ about the shock-foot position, it is argued that, as a first approximation, the variations in $\tilde{C}_{f_{0}}$ are linearly correlated with the shock-foot displacement:

$$
\tilde{C}_{f_{0}} \approx \Lambda \zeta
$$

with $\Lambda$ in the range $2 \times 10^{-3}$ to $3 \times 10^{-3}$ for the case considered. Using (4.17) and 


\begin{tabular}{|c|c|c|}
\hline Variable & Amplitude & Justification \\
\hline$\zeta$ & $O\left(10^{-1}\right)$ & Figure 2 \\
\hline$\dot{\zeta}^{\dagger}$ & $O\left(10^{-3}\right)$ & (3.2), (4.15) with $t \equiv t_{s}$ \\
\hline$k, k \tan \beta$ & $O\left(10^{-1}\right)$ & Figure $8(b), \beta \approx 32^{\circ}$ \\
\hline $\bar{C}_{f_{0}}$ & $O\left(10^{-3}\right)$ & Configuration input, figure 9 \\
\hline$C_{f_{0}}^{\prime \prime}$ & $O\left(10^{-4}\right)$ & From LES data \\
\hline$\Lambda$ & $O\left(10^{-3}\right)$ & Figure 9 \\
\hline$F^{\prime}(0)$ & $O\left(10^{-1}\right)$ & Figure 7 \\
\hline$p_{1} /\left(\rho_{1} u_{1}^{2}\right)^{\ddagger}$ & $O\left(10^{-1}\right)$ & For $\gamma=1.4$ and $M_{1}=2.3$ \\
\hline$l_{0} /(2 L)^{1}$ & $O\left(10^{-1}\right)$ & By geometry with $\alpha \approx 29^{\circ}, \beta \approx 32^{\circ}$ \\
\hline$\kappa_{1}, \kappa_{\rho}, \kappa_{p}$ & $O(1)$ & Figure $8(a)$ \\
\hline$\kappa_{2}$ & $O\left(10^{-1}\right)$ & Figure $8(a)$ \\
\hline$\Theta_{1} / L, \Theta_{2} / L$ & $O\left(10^{-2}\right)$ & Figure $8(a)$ \\
\hline$\Theta_{\rho} / L, \Theta_{p} / L$ & $O\left(10^{-1}\right)$ & Figure $8(a)$ \\
\hline
\end{tabular}

TABLE 2. Amplitudes of all the constituents found in (4.20) for $M_{1}=2.3$ and $\theta=8^{\circ}$.

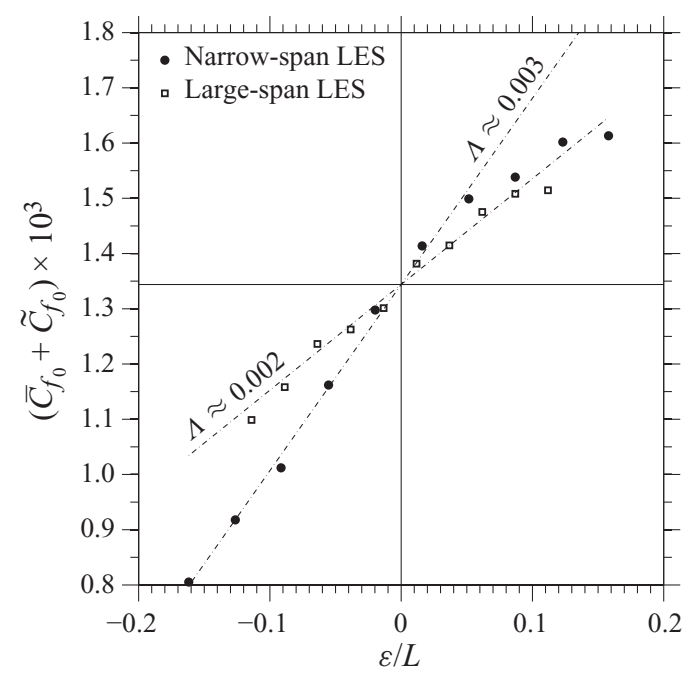

FigurE 9. Relationship between $\bar{C}_{f_{0}}+\tilde{C}_{f_{0}}$ and $\zeta=\varepsilon / L$.

(4.18) in (4.16) gives

$$
\begin{aligned}
& k \tan \beta\left(\kappa_{1}-\kappa_{\rho}\right) \zeta \dot{\zeta}+\left(\frac{\Theta_{\rho}}{L}-\frac{\Theta_{1}}{L}\right) \dot{\zeta} \\
& \quad+\left[\frac{1-k}{2 F^{\prime}(0)}\left(\bar{C}_{f_{0}}+\Lambda \zeta+C_{f_{0}}^{\prime \prime}\right)-k \tan \beta\left(\kappa_{2}+\frac{p_{1}}{\rho_{1} u_{1}^{2}} \kappa_{p}\right)\right] \zeta \\
& =\frac{l_{0}}{2 L F^{\prime}(0)}\left(\bar{C}_{f_{0}}+\Lambda \zeta+C_{f_{0}}^{\prime \prime}\right)-\frac{\Theta_{2}}{L}-\frac{p_{1}}{\rho_{1} u_{1}^{2}} \frac{\Theta_{p}}{L}
\end{aligned}
$$

Each term in (4.19) can now be quantified. This is performed in the case of an upstream Mach number $M_{1}=2.3$ and a wedge angle $\theta$ set to $8^{\circ}$, which gives $\alpha \approx 29^{\circ}$ and $\beta \approx 32^{\circ}$ from inviscid theory. The orders of magnitude of all the constituents in 
(4.19) are provided in table 2 and the governing equation is

$$
\begin{aligned}
& \underbrace{k \tan \beta}_{O\left(10^{-1}\right)}(\underbrace{\kappa_{1}}_{O(1)}-\underbrace{\kappa_{\rho}}_{O(1)}) \underbrace{\zeta \dot{\zeta}}_{O\left(10^{-4}\right)}+(\underbrace{\left[\frac{\Theta_{\rho}}{L}\right]}_{O\left(10^{-1}\right)}-\underbrace{\left[\frac{\Theta_{1}}{L}\right]}_{O\left(10^{-2}\right)}) \underbrace{\dot{\zeta}}_{O\left(10^{-3}\right)} \\
& +[(\underbrace{\bar{C}_{f_{0}}}_{O\left(10^{-3}\right)}+\underbrace{\Lambda \zeta}_{O\left(10^{-4}\right)}+\underbrace{C_{f_{0}}^{\prime \prime}}_{O\left(10^{-4}\right)}) \underbrace{\left[\frac{1-k}{2 F^{\prime}(0)}\right]}_{O(1)} \\
& -\underbrace{k \tan \beta}_{O\left(10^{-1}\right)}(\underbrace{\kappa_{2}}_{O\left(10^{-1}\right)}+\underbrace{\left[\frac{p_{1}}{\rho_{1} u_{1}^{2}} \kappa_{p}\right]}_{O\left(10^{-1}\right)})] \underbrace{\zeta}_{O\left(10^{-1}\right)} \\
& =\underbrace{\left[\frac{l_{0}}{2 L F^{\prime}(0)}\right]}_{O(1)}(\underbrace{\bar{C}_{f_{0}}}_{O\left(10^{-3}\right)}+\underbrace{\Lambda \zeta}_{O\left(10^{-4}\right)}+\underbrace{C_{f_{0}}^{\prime \prime}}_{O\left(10^{-4}\right)})-\underbrace{\left[\frac{\Theta_{2}}{L}\right]}_{O\left(10^{-2}\right)}-\underbrace{\left[\frac{p_{1}}{\rho_{1} u_{1}^{2}} \frac{\Theta_{p}}{L}\right]}_{O\left(10^{-2}\right)} .
\end{aligned}
$$

Neglecting all the $O\left(10^{-5}\right)$ terms, (4.20) reduces to

$$
\begin{aligned}
\frac{\Theta_{\rho}}{L} \dot{\zeta}+\left[\frac{1}{2 F^{\prime}(0)}\left(\bar{C}_{f_{0}}(1-k)-\frac{l_{0}}{L} \Lambda\right)\right. & \left.-k \tan \beta\left(\kappa_{2}+\frac{p_{1}}{\rho_{1} u_{1}^{2}} \kappa_{p}\right)\right] \zeta \\
= & \frac{l_{0} C_{f_{0}}^{\prime \prime}}{2 L F^{\prime}(0)}+\underbrace{\frac{l_{0} \bar{C}_{f_{0}}}{2 L F^{\prime}(0)}-\frac{\Theta_{2}}{L}-\frac{p_{1}}{\rho_{1} u_{1}^{2}} \frac{\Theta_{p}}{L}}_{\Upsilon} .
\end{aligned}
$$

Equation (4.21) is now a linear first-order ODE with both a forcing term $C_{f_{0}}^{\prime \prime}$ and a steady term $\Upsilon$ on the right-hand side. It is well known from the LES and from the experiments that the reflected shock oscillates about a mean position (in a nonharmonic manner). In other words, the reflected-shock-foot motions must be governed by a stable dynamical system and in the absence of any external forcing, the shock must remain at its equilibrium position. In the current coordinate system, this means $\zeta=0$ in the absence of any forcing (i.e. $C_{f_{0}}^{\prime \prime}=0$ ). Applying this condition to (4.21) leads to $\Upsilon=0$. Hence, the system is governed by

$$
\frac{l_{0} \bar{C}_{f_{0}}}{2 L F^{\prime}(0)}-\frac{\Theta_{2}}{L}-\frac{p_{1}}{\rho_{1} u_{1}^{2}} \frac{\Theta_{p}}{L}=0
$$

and

$$
\dot{\zeta}+\phi \zeta=\psi(t)
$$


where

$$
\begin{aligned}
\phi & =\frac{L}{\Theta_{\rho}}\left[\frac{1}{2 F^{\prime}(0)}\left(\bar{C}_{f_{0}}(1-k)-\frac{l_{0}}{L} \Lambda\right)-k \tan \beta\left(\kappa_{2}+\frac{p_{1}}{\rho_{1} u_{1}^{2}} \kappa_{p}\right)\right], \\
\psi(t) & =\frac{l_{0} C_{f_{0}}^{\prime \prime}(t)}{2 L F^{\prime}(0)} .
\end{aligned}
$$

Equation (4.22a) is the reflected-shock-foot steady-state equation whereas $(4.22 b)$ is its dynamical equation in the presence of fluctuations. Equation (4.22a) shows that, on average, the most significant balance is the balance between the rate of changes of momentum and pressure thicknesses (as previously noted in connection with figure $6 a$ ). As one could have anticipated, the error in this statement scales with the mean skin friction and is of the order of $10^{-3}$ in the present case.

Quite remarkably, (4.22b) is similar to the model proposed by Plotkin (1975), and the above may be viewed as a derivation of his model. This is discussed in more detail later. Generally speaking, $(4.22 b)$ is a stochastic differential equation resembling a Langevin equation for Brownian motions (see chapter 3 of Risken 1989), where $\phi$ is the damping coefficient and $\psi(t)$ is the Langevin force, with zero mean (i.e. $\overline{\psi(t)}=0$ ). However, the main difference with the classical Langevin equation resides in the time-correlation properties of the forcing, which is not proportional to a Dirac function, at least for time scales of the order of $\delta_{0} / \bar{u}_{1}$ (we shall see that for the time scales considered in the present problem, i.e. $O\left(10^{2} \delta_{0} / \bar{u}_{1}\right)$, the forcing may be considered similar to a white noise). One interesting property of $(4.22 b)$ is that it is sufficient to know the time correlation of the forcing (i.e. $\left.\overline{C_{f_{0}}^{\prime \prime}(t) C_{f_{0}}^{\prime \prime}(t+\tau)}\right)$ to calculate the correlation function of $\zeta$ (i.e. $\overline{\zeta(t) \zeta(t+\tau)}$ ), which is our ultimate goal. Therefore, if the time-correlation function of the skin-friction turbulence-related fluctuations is known, and provided that one can calculate the damping factor $\phi$, the derived governing equation $(4.22 b)$ is sufficient to predict the shock-motion low-frequency spectrum.

In the present case, the aforementioned results (i.e. $\Theta_{\rho} / L=-0.104, F^{\prime}(0)=0.12$, $\bar{C}_{f_{0}}=1.35 \times 10^{-4}, \quad k=0.32, \quad l_{0} / L=\tan \beta /(\tan \alpha+\tan \beta)=0.55, \quad \Lambda=3 \times 10^{-3}$, $k \tan \beta=0.2, \kappa_{2}=0.27, \kappa_{p}=-1.23$ and $\left.p_{1} /\left(\rho_{1} u_{1}^{2}\right)=0.134\right)$ can be used to find that the damping factor $\phi$ is roughly 0.23 . It will be shown later that the premultiplied spectrum of $\zeta$ when subject to a white-noise forcing is broadband with a peak at $\phi /(2 \pi)$. For $\phi \approx 0.23$, one finds $\phi /(2 \pi) \approx 0.037$, which is reminiscent of the Strouhalnumber value observed in the LES-weighted spectrum (see Touber \& Sandham $2009 b$ ). However, before discussing this encouraging result further, it is of importance to try to model the coefficients $\Theta_{\rho}, k, \kappa_{2}$ and $\kappa_{p}$ to overcome the need for some prior LES results.

\section{Modelling the ODE coefficients}

\subsection{The $k$ coefficient}

If the shock maintains its inclination angle at all times, one could write $s=\varepsilon \tan \alpha /(\tan \alpha+\tan \beta)$. However, this is not the case. For example, Dupont et al. (2006) noted that 'the reflected shock appears as a low-frequency unsteady sheet with a length of excursion vanishing far from the wall'. This implies that the reflected shock angle with respect to the wall changes as the shock moves back and forth. This picture may also be observed in the conditional averages, as shown in figure 3 and also in side-view animations of the LES (see Touber \& Sandham 2010). At 


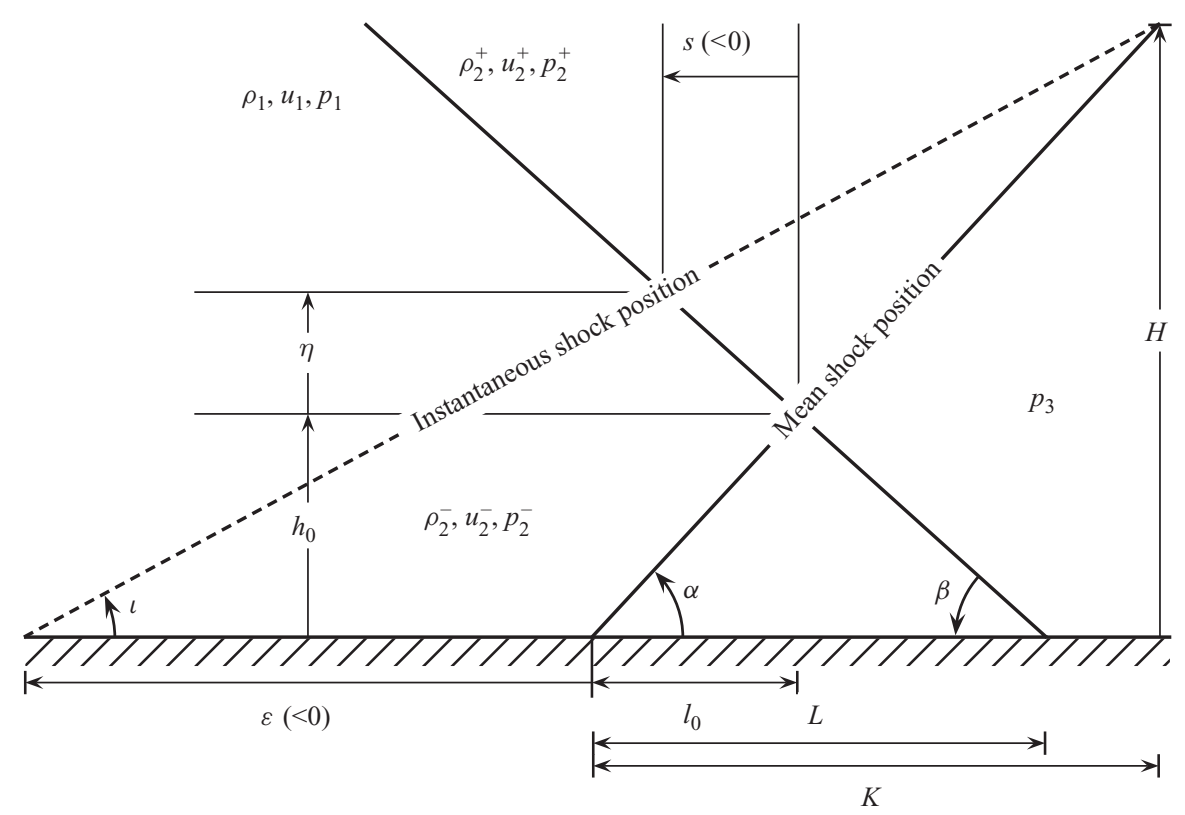

FIGURE 10. Sketch of the interaction with the notations used to compute $k$.

high frequencies, the shock cannot be considered to be a straight line (or sheet) because of transverse travelling waves, and defining an inclination angle may be difficult and not meaningful. However, at sufficiently low frequencies, the reflected shock appears to move as a whole and may be thought of a straight line (or sheet) with a given foot position and inclination angle (see Touber \& Sandham 2010). The quantitative relationship between $s$ and $\varepsilon$ seen in figure $8(b)$ can be combined with the aforementioned comments to justify relating $s$ and $\varepsilon$ as in (4.13), if the study is restricted to the low-frequency motions. An analytical expression for $k$ in (4.13) is derived in the following paragraph.

From the notation defined in figure 10 , one can write the following geometric relations:

to find that

$$
\left.\begin{array}{l}
K=H / \tan \alpha, \\
K-\varepsilon=H / \tan \iota, \\
\tan \iota=\left(h_{0}+\eta\right) /\left(l_{0}-\varepsilon+s\right), \\
\eta=-s \tan \beta, \\
\tan \alpha=h_{0} / l_{0},
\end{array}\right\}
$$

$$
s=\frac{\left(\varepsilon / h_{0}\right)\left(h_{0}-H\right)}{\left(\varepsilon / h_{0}\right) \tan \beta-\left(H / h_{0}\right)\left(1+\frac{\tan \beta}{\tan \alpha}\right)} .
$$

Assuming $\varepsilon / h_{0} \ll 1$, one can expand the above equation in series of $\varepsilon / h_{0}$ to find

$$
s=\frac{H / h_{0}-1}{\left(H / h_{0}\right)\left(1+\frac{\tan \beta}{\tan \alpha}\right)} \varepsilon+O\left(\varepsilon^{2} / h_{0}\right) .
$$


Following the aforementioned remark by Dupont et al. (2006), which is supported by figure 3 , one can say that $H$ is significantly larger than the incoming boundarylayer height $\delta_{0}$. One way to look at $H$ is to see it as a penetration length scale corresponding to the wall-normal distance a perturbation associated with a shockfoot displacement can travel along the shock during half of a low-frequency cycle. If $V$ is the propagation speed and $T$ is the typical period of a low-frequency cycle, one could write $H=(T / 2) V \sin \alpha$. Roughly, the propagation speed can be thought to scale with $u_{1} \cos \alpha$ and the characteristic frequency of the system with $u_{1} / L$, so that $H \sim \pi L \sin \alpha \cos \alpha=L \pi \sin (2 \alpha) / 2$. Considering that $\pi \sin (2 \alpha) / 2 \sim 1$, one finds that $H \sim L$. Therefore, one could replace $H$ with $L$ in $(5.3 a)$ and write

$$
s \approx \frac{L / h_{0}-1}{\left(L / h_{0}\right)\left(1+\frac{\tan \beta}{\tan \alpha}\right)} \varepsilon .
$$

Noting the geometrical relation $h_{0} / L=\tan \alpha \tan \beta /(\tan \alpha+\tan \beta)$, the theoretical value for $k$ is

$$
k=\frac{1-\tan \alpha \tan \beta /(\tan \alpha+\tan \beta)}{1+\tan \beta / \tan \alpha} .
$$

For $M_{1}=2.3$ and a wedge angle of $8^{\circ}$, inviscid theory gives that $\beta \approx 32.4^{\circ}$ and $\alpha \approx 29.4^{\circ}$, so that the theoretical $k$ value is about 0.33 , to compare with 0.38 in the large-span LES and 0.32 in the narrow-span LES (see figure $8 b$ ). The average error is less than $6 \%$ and therefore (5.4) is considered a good first-order approximation.

\subsection{The $\Theta_{i}$ coefficients}

The $\Theta$ coefficients represent the mean changes of thicknesses (i.e. $\delta_{1}, \delta_{2}, \delta_{\rho}, \delta_{p}$ ) between the shock foot $\xi=0$ and the shock-crossing point $\xi=1$. Note that, rigorously, we should write $\xi=1^{-}$owing to the discontinuity at this station. However, the thicknesses being integral quantities, the presence of the discontinuity is in fact irrelevant and we can write $\xi=1$. Although such quantities are not generally known, this section will introduce a model to estimate $\Theta_{p}$ and show how it can be related to $\Theta_{\rho}$ and $\Theta_{2}$ in a useful way.

In canonical boundary layers, the pressure is considered constant in the wall-normal direction. In the presence of the oblique shock, this approximation is obviously inadequate. However, upstream of the interaction, the boundary layer is a typical turbulent boundary layer and one can write

$$
\bar{p}(\xi<0, y) \approx p_{1},
$$

which is easily verified from figure 11 . Inside the interaction, the picture is more complex. At $\xi=1$ and $y=h_{0}$, the pressure is discontinuous, jumping from $p_{1}$ to $\bar{p}_{3}$ (where $\bar{p}_{3}$ refers to the mean pressure downstream of the interaction) whereas at the wall, the pressure continuously increases from $p_{1}$ to $\bar{p}_{3}$ over a streamwise distance ranging from $\xi \approx 0$ to well beyond the reattachment point. However, on the basis of figure 11, we argue that the isobar in the vicinity of $\xi=1$ can be modelled as a straight line given its actual ' $\mathrm{S}$ ' shape in the figure (see white dots), with the straight line chosen such that it averages the S. From the data, this idealised isobar would take a value between $p_{2}^{+}$and $\bar{p}_{3}$ (see the dash-dotted isobar corresponding to $p_{2}^{+}$); hence

$$
\bar{p}(\xi=1, y) \approx(1-r) p_{2}^{+}+r \bar{p}_{3},
$$


(a)

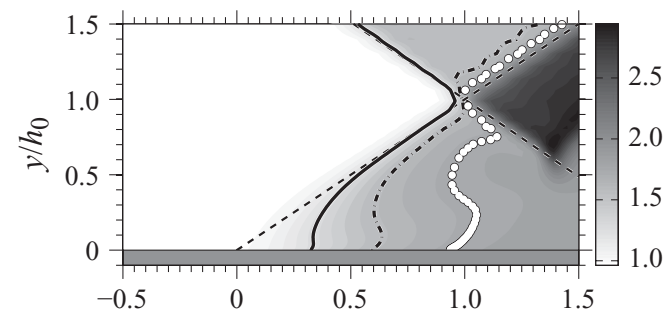

(c)

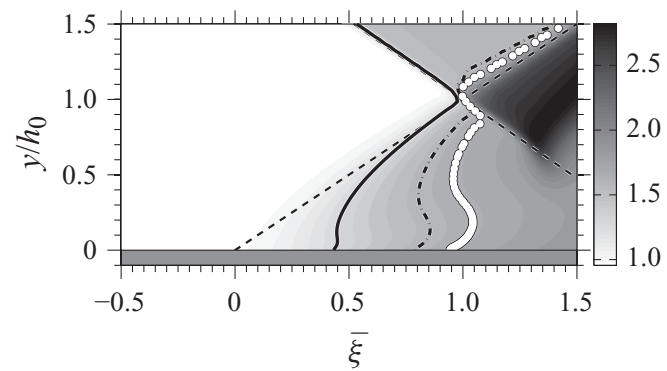

(b)

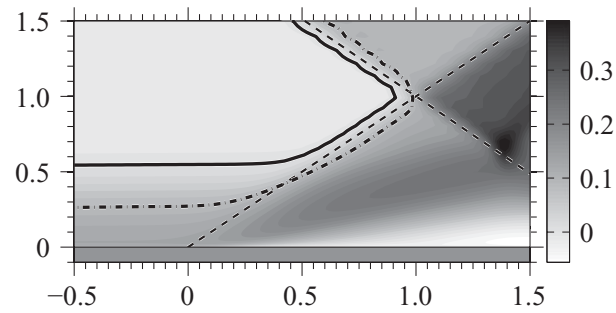

(d)

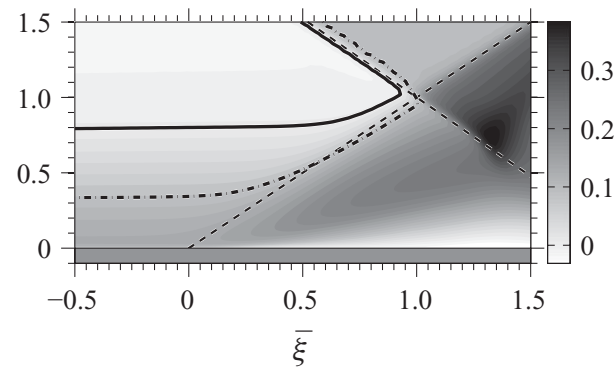

Figure 11. Mean pressure field $\bar{p} / p_{1}(a, c)$ and the mean momentum-thickness-integrand field $\left[\overline{\rho u} /\left(\rho_{1} u_{1}\right)-\overline{\rho u u} /\left(\rho_{1} u_{1} u_{1}\right)\right](b, d)$. Both the $(a, b)$ narrow-span and $(c, d)$ large-span LES data are shown. The thick solid and dash-dotted lines indicate the contours equal to the inviscid-theory results, using the upper-region values (e.g. $p_{2}^{+}, \rho_{2}^{+}, u_{2}^{+}$) for the dash-dotted lines and the bottom-region values (e.g. $p_{2}^{-}, \rho_{2}^{-}, u_{2}^{-}$) for the solid lines. The shock-system location is indicated by the set of dashed lines. The white dots in the pressure fields show the contours $(1-r) p_{2}^{+} / p 1+r \bar{p}_{3} / p_{1}$ with $r=0.2$ and 0.1 for $(a)$ and $(c)$, respectively.

with $r$ being a weighting factor. In the present case, $r \approx 0.2$ gives satisfactory results. Assuming the distributions (5.5) and (5.6), one finds

$$
\begin{aligned}
\frac{\Theta_{p}}{L} & \equiv \frac{1}{L}\left[\int_{0}^{h_{0}}\left(1-\frac{\bar{p}\left(\xi=1^{-}, y\right)}{p_{1}}\right) \mathrm{d} y-\int_{0}^{h_{0}}\left(1-\frac{\bar{p}(\xi=0, y)}{p_{1}}\right) \mathrm{d} y\right] \\
& \approx\left\{1-(1-r) \frac{p_{2}^{+}}{p_{1}}-r \frac{\bar{p}_{3}}{p_{1}}\right\} \frac{\tan \alpha \tan \beta}{\tan \alpha+\tan \beta},
\end{aligned}
$$

noting from geometrical considerations in figure 10 that $h_{0} / L=\tan \alpha \tan \beta /(\tan \alpha+$ $\tan \beta$ ). Applying (5.7) to the $M_{1}=2.3$ and $8^{\circ}$-wedge-angle case gives $\Theta_{p} / L=-0.233$ (with $r=0.2$ ) to compare with the LES value of -0.243 from figure $8(a)$. Of course, the choice of $r$ was based on the LES data whereas, generally, one has no prior knowledge of this value. However, note that the existence of a similarity function (see figure $7 c$ ) suggests that the weighting factor $r$ does not change during the shock motions and can thus be treated as a true constant.

In the previous section it was shown that, on average, the changes of pressure and momentum thicknesses between $\xi=0$ and $\xi=1$ are close to equilibrium with an error scaling on the skin friction (see $(4.22 a)$ ). Therefore, as a first approximation:

$$
\Theta_{2} \approx-\frac{p_{1}}{\rho_{1} u_{1}^{2}} \Theta_{p}, \text { or equivalently } \frac{\Theta_{2}}{\Theta_{p}} \approx-\frac{1}{\gamma M_{1}^{2}},
$$

where the ideal-gas law was used to transform $p_{1} /\left(\rho_{1} u_{1}^{2}\right)$ in $1 /\left(\gamma M_{1}^{2}\right)$. Using (5.8) with $M_{1}=2.3$ and $\gamma=1.4$, one finds $\Theta_{2} / \Theta_{p}=-0.135$, whereas from figure $8(a)$, the LES 
(a)

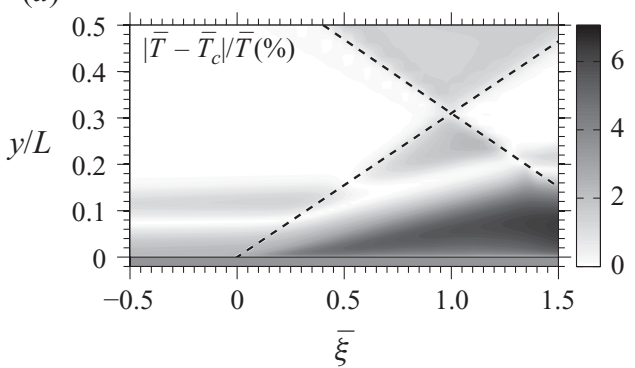

(b)

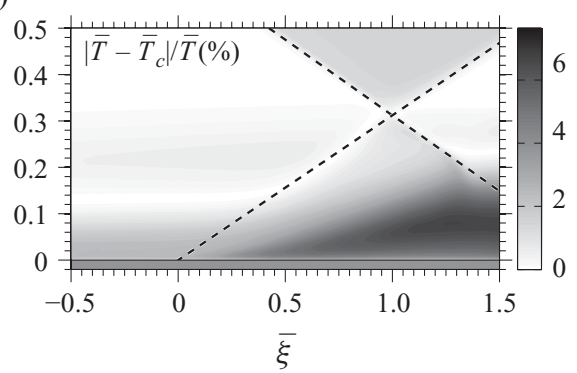

FIGURE 12. Percentage error between the temperature field computed from the velocity field using the Crocco-Busemann equation and the actual LES temperature field for the $(a)$ narrow-span and $(b)$ large-span simulations.

gives -0.132 , which is a satisfactory agreement. This result confirms that the error in (5.8) scales with $l_{0} \bar{C}_{f_{0}} /\left(2 L F^{\prime}(0)\right) \approx 3 \times 10^{-3}$, providing an encouraging consistency check.

Finally, to relate $\Theta_{\rho}$ to $\Theta_{p}$, the Crocco-Busemann relation will be used (see White 1991). The temperature field computed using the Crocco-Busemann relation is denoted by $T_{c}$ and defined as

$$
\frac{T_{c}}{T_{1}}=1+\frac{\gamma-1}{2} M_{1}^{2}\left(\frac{u}{u_{1}}\right)^{2},
$$

assuming the wall to be isothermal and equal to the upstream adiabatic-wall condition (as in the current LES settings). The validity of (5.9) is tested using the LES results and the error contour levels are shown in figure 12. Overall, the use of (5.9) is remarkably accurate with errors not exceeding $7 \%$.

Starting from the definition of the pressure thickness and using the ideal-gas law, one finds

$$
\delta_{p}=\int_{0}^{h}\left(1-\frac{p}{p_{1}}\right) \mathrm{d} y=\int_{0}^{h}\left(1-\frac{\rho T}{\rho_{1} T_{1}}\right) \mathrm{d} y,
$$

which with (5.9) may be approximated by

$$
\delta_{p} \approx \int_{0}^{h}\left\{1-\frac{\rho}{\rho_{1}}\left[1+\frac{\gamma-1}{2} M_{1}^{2}\left(1-\frac{u^{2}}{u_{1}^{2}}\right)\right]\right\} \mathrm{d} y,
$$

which can be expressed in terms of the density, displacement and momentum thickness definitions:

$$
\delta_{p} \approx \delta_{\rho}\left(1+\frac{\gamma-1}{2} M_{1}^{2}\right)-\frac{\gamma-1}{2} M_{1}^{2}\left(\delta_{1}+\delta_{2}\right) .
$$

Using (5.10c) in the definition of $\Delta_{i}$ (see (4.8)) and time-averaging, one finds

$$
\Theta_{p} \approx \Theta_{\rho}\left(1+\frac{\gamma-1}{2} M_{1}^{2}\right)-\frac{\gamma-1}{2} M_{1}^{2}\left(\Theta_{1}+\Theta_{2}\right) .
$$

Using (5.8), (5.11a) becomes

$$
\Theta_{p} \approx \Theta_{\rho}\left(1+\frac{\gamma-1}{2} M_{1}^{2}\right)-\frac{\gamma-1}{2} M_{1}^{2}\left(\Theta_{1}-\frac{1}{\gamma M_{1}^{2}} \Theta_{p}\right) .
$$


From figure $8(a)$, it is found that for the configuration studied here $\left|\Theta_{p} /\left(\gamma L M_{1}^{2}\right)\right| \approx$ $3 \times 10^{-2}$ whereas $\Theta_{1} / L \approx 6 \times 10^{-3}$. Therefore, as a leading-order approximation, the term in $\Theta_{1}$ in $(5.11 b)$ may be neglected:

$$
\Theta_{p} \approx \Theta_{\rho}\left(1+\frac{\gamma-1}{2} M_{1}^{2}\right)+\frac{\gamma-1}{2 \gamma} \Theta_{p} .
$$

Equation $(5.11 c)$ is re-arranged:

$$
\Theta_{p} \approx \chi \Theta_{\rho}, \quad \text { with } \quad \chi=\frac{2 \gamma+\gamma(\gamma-1) M_{1}^{2}}{\gamma+1} .
$$

For $M_{1}=2.3,(5.11 d)$ gives $\chi=2.40$ while the LES data in figure $8(a)$ give $\chi=2.34$. It should be stressed that the Crocco-Busemann equation used to derive $(5.11 d)$ was assumed to be applicable to the unsteady velocity field, the displacement-thickness contribution was neglected and the momentum-thickness contribution was related to the pressure thickness using (5.8). Despite those gross assumptions, only a $3 \%$ difference with the LES can be found for the present configuration.

\subsection{The $\kappa_{p}$ and $\kappa_{2}$ coefficients}

By definition, the $\kappa_{p}$ and $\kappa_{2}$ coefficients in (4.11) correspond to the rate of change of the pressure and momentum thickness between $\xi=1$ and $\xi=0$ as the shock moves back and forth (i.e. $\kappa_{p}=\mathrm{d} \Delta_{p} / \mathrm{d} \eta, \kappa_{2}=\mathrm{d} \Delta_{2} / \mathrm{d} \eta$ ). Although not explicitly written in (4.11), the shock motions of interest for this study are the low-frequency ones. Therefore, the reflected shock is considered to remain straight and to rotate around its foot as it oscillates. One direct consequence of such a motion is a modification of the pressure field in the region $2^{-}$as well as in region 3 (i.e. $p_{2}^{-}$and $p_{3}$ in figure 10 ), whereas $p_{2}^{+}$remains unchanged. We wish to express those changes in terms of the variable $\eta$, which is made possible by developing a series expansion of the classical oblique-shock jump relations, considering that $\eta / h_{0}$ is sufficiently small. The details of such expansions are provided in Appendix $\mathrm{C}$ and only the final result is reported here. For the pressure in region 3, we find

$$
\frac{p_{3}}{p_{1}}=\frac{\bar{p}_{3}}{p_{1}}+\frac{p_{2}^{+}}{p_{1}} \frac{\gamma M_{2}^{2} \varkappa}{1+\gamma} \frac{\eta}{h_{0}}+O\left(\frac{\eta^{2}}{h_{0}^{2}}\right)
$$

where $\chi=(\tan \alpha+\tan \beta) \sin (2 \alpha) \sin [2(\alpha+\theta)] /(\tan \beta(1-1 / \tan \alpha)-1)$. Assuming that the distributions (5.5) and (5.6) can be extented to the low-frequency oscillations, it is possible to write

$$
\Delta_{p}=\left[1-(1-r) \frac{p_{2}^{+}}{p 1}-r \frac{p_{3}}{p_{1}}\right]\left(h_{0}+\eta\right) .
$$

Using the series expansion (5.12) and (5.7), the above equation becomes

$$
\Delta_{p}=\Theta_{p}+\left\{1-r \frac{\bar{p}_{3}}{p_{1}}-\frac{p_{2}^{+}}{p_{1}}\left[1-r\left(1+\frac{\gamma M_{2}^{2} \varkappa}{1+\gamma}\right)\right]\right\} \eta+O\left(\frac{\eta^{2}}{h_{0}}\right) .
$$

Thus,

$$
\begin{aligned}
\kappa_{p} \approx 1- & r \frac{\bar{p}_{3}}{p_{1}}-\frac{p_{2}^{+}}{p_{1}}\left[1-r\left(1+\frac{\gamma M_{2}^{2} \varkappa}{1+\gamma}\right)\right], \\
& \text { with } x=\frac{\tan \alpha+\tan \beta}{\tan \beta(1-1 / \tan \alpha)-1} \sin (2 \alpha) \sin [2(\alpha+\theta)], \quad r=0.2 .
\end{aligned}
$$



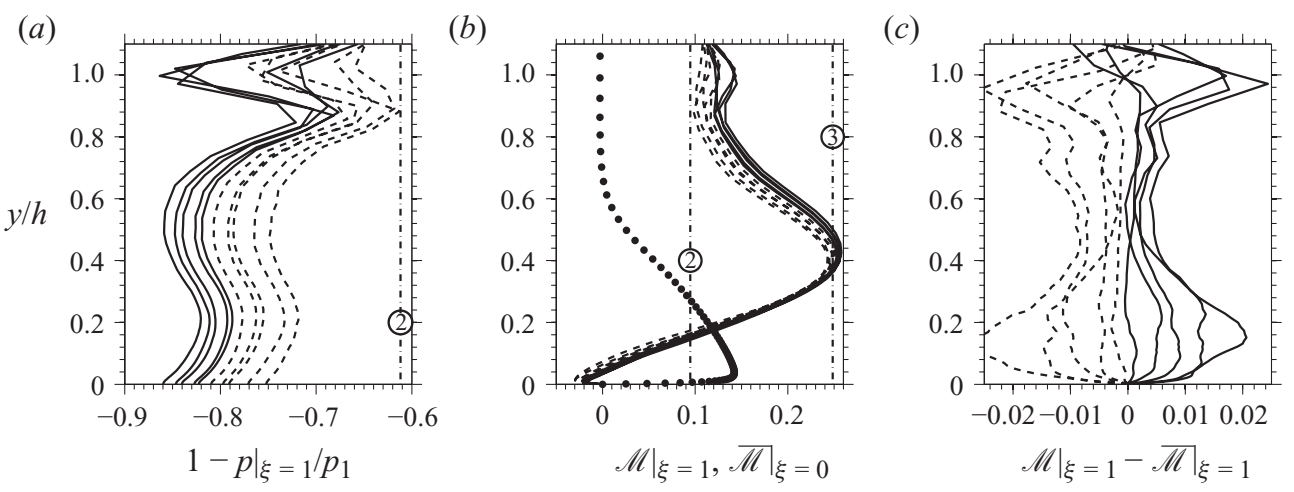

Figure 13. Integrands of $\delta_{p}$ and $\delta_{2}$ at $\xi=1$. The solid lines indicate cases where $\eta>0$ whereas dashed lines indicate cases where $\eta<0$. In $(b)$, the dotted line corresponds to $\overline{\mathscr{M}}$ at $\xi=0$. The numbers 2 and 3 embedded in black circles indicate the potential-flow values in regions 2 and 3 , respectively.

For $M_{1}=2.3$ and $\theta=8^{\circ}$, the modelled $\kappa_{p}$ using (5.14) gives -1.21 whereas the measured value on the narrow-span LES data is -1.23 . The difference is thus less than $2 \%$ for the particular choice of weighting factor $r=0.2$. As mentioned earlier, the weighting factor is based on time-averaged LES data and its use here is justified based on the similarity hypothesis, combined with (5.6) and the above series expansion.

The case of $\kappa_{2}$ is more complex than $\kappa_{p}$ mainly because the quantity $\mathscr{M} \equiv \rho u(1-$ $\left.u / u_{1}\right) /\left(\rho_{1} u_{1}\right)$ at $\xi=0$ and $\xi=1$ is not constant along the wall-normal direction, even approximately, as shown in figures 11 and $13(b)$. From the definition of the momentum thickness $(4.2 b)$, we have

$$
\frac{\Delta_{2}}{h}=\underbrace{\left.\frac{1}{h} \int_{0}^{h} \mathscr{M}\right|_{\xi=1} \mathrm{~d} y}_{\delta_{2, \xi=1} / h}-\underbrace{\left.\frac{1}{h} \int_{0}^{h} \mathscr{M}\right|_{\xi=0} \mathrm{~d} y}_{\delta_{2, \xi=0} / h} .
$$

Decomposing $\mathscr{M}(\xi=1, y)$ in its steady $(\overline{\mathscr{M}}(\xi=1, y))$ and time-dependent $\left(\mathscr{M}^{\prime}(\xi=1, y)\right)$ component, one can write

$$
\frac{\delta_{2, \xi=1}}{h}=\left.\frac{1}{h} \int_{0}^{h} \overline{\mathscr{M}}\right|_{\xi=1} \mathrm{~d} y+\left.\frac{1}{h} \int_{0}^{h} \mathscr{M}^{\prime}\right|_{\xi=1} \mathrm{~d} y .
$$

Since $\overline{\mathscr{M}}(\xi=1, y)$ is a continuous function on $y \in[0, h[$, the mean-value theorem states that there exists a positive real number $R$ such that

$$
\left.\frac{1}{h} \int_{0}^{h} \overline{\mathscr{M}}\right|_{\xi=1} \mathrm{~d} y=(1-R) \min _{0 \leqslant y<h}\left[\left.\overline{\mathscr{M}}\right|_{\xi=1}\right]+R \max _{0 \leqslant y<h}\left[\left.\overline{\mathscr{M}}\right|_{\xi=1}\right], \quad R>0 .
$$

Let us denote by $\mathscr{M}_{3}$ and $\overline{\mathscr{M}}_{3}$ the following quantities, respectively:

$$
\mathscr{M}_{3} \equiv \frac{\rho_{3} u_{3}}{\rho_{1} u_{1}}\left(1-\frac{u_{3}}{u_{1}}\right), \quad \overline{\mathscr{M}}_{3} \equiv \frac{\bar{\rho}_{3} \bar{u}_{3}}{\rho_{1} u_{1}}\left(1-\frac{\bar{u}_{3}}{u_{1}}\right) .
$$

From figure $13(b)$ one can see that $\left.\overline{\mathscr{M}}\right|_{\xi=1}$ does not exceed $\overline{\mathscr{M}}_{3}$, whereas inside the separation bubble, it is possible that it becomes negative. Recalling that the separation bubble height is very small compared to $h$ (see Touber $\&$ Sandham 2009a), it is argued 
that the possible negative contribution of $\overline{\mathscr{M}}$ in (5.17) remains small so that (5.17) becomes

$$
\left.\frac{1}{h} \int_{0}^{h} \overline{\mathscr{M}}\right|_{\xi=1} \mathrm{~d} y=R \overline{\mathscr{M}}_{3}
$$

Similarly, the fluctuating component $\left.\mathscr{M}^{\prime}\right|_{\xi=1}$ (shown in figure 13c) is related to $\mathscr{M}_{3}^{\prime}$ by invoking the mean-value theorem:

$$
\left.\frac{1}{h} \int_{0}^{h} \mathscr{M}^{\prime}\right|_{\xi=1} \mathrm{~d} y=r^{\prime}\left(\mathscr{M}_{3}-\overline{\mathscr{M}}_{3}\right)
$$

where $r^{\prime}$ is a real number. As for the pressure $p_{3}$, when the reflected shock moves back and forth, $\mathscr{M}_{3}$ will fluctuate and those changes can be expressed in terms of a series expansion in $\eta / h_{0}$ :

$$
\mathscr{M}_{3}=\overline{\mathscr{M}}_{3}+D \frac{\eta}{h_{0}}+O\left(\frac{\eta^{2}}{h_{0}^{2}}\right)
$$

where $D$ is a constant defined in (C 18) in Appendix C. Using (5.16), (5.19) and (5.20) in (5.15) gives

$$
\Delta_{2}=\underbrace{R \overline{\mathscr{M}}_{3} h_{0}-\bar{\delta}_{2, \xi=0}}_{\Theta_{2}}+\underbrace{\left(R \overline{\mathscr{M}}_{3}+r^{\prime} D\right)}_{\kappa_{2}} \eta+O\left(\frac{\eta^{2}}{h_{0}}\right),
$$

assuming that the fluctuations of the momentum thickness at $\xi=0$ are negligible compared with the variations at $\xi=1$ (i.e. $\tilde{\delta}_{2, \xi=0} \approx 0$ ) and where terms $\Theta_{2}$ and $\kappa_{2}$ are identified according to (4.11). To be consistent with (5.8), we must take $R$ such that

$$
R=\frac{1}{\overline{\mathscr{M}}_{3}}\left(\frac{\bar{\delta}_{2, \xi=0}}{h_{0}}-\frac{1}{\gamma M_{1}^{2}} \frac{\Theta_{p}}{h_{0}}\right) .
$$

The term in $\bar{\delta}_{2, \xi=0}$ is problematic since the momentum thickness at the shock foot is different from that of the incoming boundary layer and therefore needs to be modelled. The noticeable linear relationship between $L / \delta_{0}$ and $\left(p_{2}-p_{1}\right) /\left(2 \tau_{w}\right)$ in figure 7 of Touber \& Sandham (2009a) suggests that the momentum thickness at the shock foot may be related to the pressure jump $p_{2}-p_{1}$ as follows:

$$
\frac{\bar{\delta}_{2, \xi=0}}{h_{0}}=r^{\prime \prime} \frac{2 \tau_{w}}{p_{2}^{+}-p_{1}} \frac{L}{h_{0}},
$$

where the ratio $\bar{\delta}_{2, \xi=0} / \delta_{0}$ was considered constant and $r^{\prime \prime}$ is a positive real number to be determined from the LES data. Noting that $2 \tau_{w}=\bar{C}_{f_{0}} p_{1} \gamma M_{1}^{2}$ and $h_{0} / L=\tan \alpha \tan \beta /(\tan \alpha+\tan \beta)$, one finds

$$
\frac{\bar{\delta}_{2, \xi=0}}{h_{0}}=r^{\prime \prime} \frac{\gamma M_{1}^{2} \bar{C}_{f_{0}}}{p_{2}^{+} / p_{1}-1} \frac{\tan \alpha+\tan \beta}{\tan \alpha \tan \beta} .
$$

Hence, the following expression for $\kappa_{2}$ :

$$
\kappa_{2}=r^{\prime \prime} \frac{\gamma M_{1}^{2} \bar{C}_{f_{0}}}{p_{2}^{+} / p_{1}-1} \frac{\tan \alpha+\tan \beta}{\tan \alpha \tan \beta}-\frac{1}{\gamma M_{1}^{2}} \frac{\Theta_{p}}{h_{0}}+r^{\prime} D .
$$

The factors $r^{\prime}$ and $r^{\prime \prime}$ are computed from the LES. Using the narrow-span LES results, one finds $r^{\prime}=-0.14$ and $r^{\prime \prime}=0.2$ (giving $\kappa_{2} \approx 0.27$ ). Similar to $r$ in $(5.7), r^{\prime}$ is assumed 
to remain constant in time. This completes the modelling of the unknowns $k, \Theta_{\rho}, \kappa_{2}$ and $\kappa_{1}$ in (4.22).

\section{Closed form of the low-order stochastic model and some solutions}

\subsection{Final form of the model}

Upon substituting (5.4), (5.7), (5.11d), (5.14) and (5.24) into (4.22), one can write the following closed form of the model:

$$
\frac{1}{\bar{u}_{1}} \frac{\mathrm{d} \varepsilon}{\mathrm{d} t}+\phi \frac{\varepsilon}{L}=\Pi C_{f_{0}}^{\prime \prime}(t)
$$

with

$$
\begin{aligned}
& \Pi=\frac{\tan \beta}{2 F^{\prime}(0)(\tan \alpha+\tan \beta)}, \\
& \phi=\frac{2 \gamma+\gamma(\gamma-1) M_{1}^{2}}{(\gamma+1)\left[1+(1-r) P_{2}-r P_{3}\right]}\left\{\Pi \left[\left(\frac{1}{\tan \alpha}+\frac{1}{\tan \beta}\right)\left(\bar{C}_{f_{0}}-\Lambda\right)\right.\right. \\
& \left.+\bar{C}_{f_{0}} \frac{\tan \alpha}{\tan \beta}\right]+\left(1-\frac{\tan \alpha \tan \beta}{\tan \alpha+\tan \beta}\right)\left[r^{\prime \prime} \frac{\gamma M_{1}^{2} \bar{C}_{f_{0}}}{P_{2}-1}-r^{\prime} D\right. \\
& \left.\left.-r \frac{P_{2} \varkappa}{\gamma+1}\left(\frac{M_{2}}{M_{1}}\right)^{2}\right]\right\} \\
& \varkappa=\frac{\tan \alpha+\tan \beta}{\tan \beta(1-1 / \tan \alpha)-1} \sin (2 \alpha) \sin [2(\alpha+\theta)], \\
& D=\frac{\bar{M}_{3}}{M_{1}}\left\{\left(\frac{1}{2} \sqrt{\frac{R_{3}}{P_{3}}}-\frac{\bar{M}_{3}}{M_{1}}\right) A+\frac{1}{2} \sqrt{\frac{P_{3}}{R_{3}}} B+\left(\frac{M_{1}}{\bar{M}_{3}} \sqrt{R_{3} P_{3}}-2 P_{3}\right) C\right\}, \\
& A=\frac{\gamma \varkappa M_{2}^{2}}{1+\gamma} P_{2} \\
& B=\varkappa\left[\frac{1}{2 \sin ^{2}(\alpha+\theta)}-\frac{(\gamma-1) M_{2}^{2}}{4+2(\gamma-1) M_{2}^{2} \sin ^{2}(\alpha+\theta)}\right] R_{3}, \\
& C=\frac{\bar{M}_{3}}{M_{1}}\left\{x\left[\frac{(\gamma-1) M_{2}^{2}}{8+4(\gamma-1) M_{2}^{2} \sin ^{2}(\alpha+\theta)}-\frac{\gamma M_{2}^{2}}{2(1-\gamma)+4 \gamma M_{2}^{2} \sin ^{2}(\alpha+\theta)}\right]\right. \\
& \left.-\frac{(\tan \alpha+\tan \beta) \cos ^{2} \alpha}{\tan \beta(1-1 / \tan \alpha)-1}\right\}
\end{aligned}
$$

where $\alpha, \beta, P_{2} \equiv p_{2}^{+} / p_{1}, P_{3} \equiv \bar{p}_{3} / p_{1}, R_{3} \equiv \bar{\rho}_{3} / \rho_{1}, M_{2}$ and $\bar{M}_{3}$ are computed from the inviscid shock-reflection problem for a given pair of wedge angle $\theta$ and upstream Mach number $M_{1}$. Factors $F^{\prime}(0), r, r^{\prime}$ and $r^{\prime \prime}$ are assumed to take the values of 0.12 , $0.2,-0.14$ and 0.2 , respectively. The term $\bar{C}_{f_{0}}$ is an input parameter, together with the upstream Mach number $M_{1}$ and wedge angle $\theta$. The coefficient $\Lambda$, although of the same order as $\bar{C}_{f_{0}}$, is not an input parameter and is not generally known. In this work, it is taken to be $3 \times 10^{-3}$ (from the LES). The term $C_{f_{0}}^{\prime \prime}$ corresponds to the skin-friction turbulence-related variations at the reflected shock foot and therefore constitutes the dynamical-system input signal. 
6.2. Solution for white-noise forcing: shock-foot and pressure spectra

As mentioned earlier, the model system $(6.1 a-h)$ is a first-order linear stochastic differential equation resembling the Langevin equation for Brownian motion, written as

$$
\dot{\varepsilon}+\Phi \varepsilon=\Gamma(t),
$$

where in the present case $\Phi=\bar{u}_{1} \phi / L$ and $\Gamma=\bar{u}_{1} \Pi C_{f_{0}}^{\prime \prime}$. If $\Gamma$ is taken to be a Langevin force with zero mean and with a correlation function proportional to a Dirac function $(\delta)$,

$$
\overline{\Gamma(t)}=0, \quad \text { and } \quad \overline{\Gamma(t) \Gamma\left(t^{\prime}\right)}=q \delta\left(t-t^{\prime}\right),
$$

with $q$ being the proportionality coefficient, then (6.2a) is equivalent to (3.1) in Risken (1989) and the system $(6.2 a, b)$ can be solved with the correlation function of the shock displacement $\varepsilon$ given by (see (3.9) in Risken 1989)

$$
\overline{\varepsilon\left(t_{0}\right) \varepsilon\left(t_{0}+\tau\right)}=\varepsilon^{2}\left(t_{0}\right) \mathrm{e}^{-\Phi\left(2 t_{0}+\tau\right)}+\frac{q}{2 \Phi}\left[\mathrm{e}^{-\Phi|\tau|}-\mathrm{e}^{-\Phi\left(2 t_{0}+\tau\right)}\right]
$$

where $t_{0}$ is a chosen time and $\tau$ is the time lag separating the actual time from $t_{0}$. To remain general, $\tau$ can be taken both positive and negative. If $t_{0}$ is taken such that $\varepsilon\left(t_{0}\right)=0,(6.3 a)$ becomes

$$
\overline{\varepsilon\left(t_{0}\right) \varepsilon\left(t_{0}+\tau\right)}=\frac{q}{2 \Phi} \mathrm{e}^{-\Phi|\tau|}\left[1-\mathrm{e}^{-2 \Phi t_{0}}\right] .
$$

Note that, dimensionally speaking, the damping coefficient $\Phi \equiv 1 / \tau_{s}$ is the inverse of a time so that $\Phi t_{0}$ is the ratio between $t_{0}$ and the system characteristic time scale $\tau_{s}$. If $t_{0}$ is chosen long after the initial transients from starting up the flow, $t_{0}$ will be significantly larger than $\tau_{s}$, so that $\Phi t_{0} \gg 1$. The autocorrelation function of the shock-foot motions in response to a white-noise forcing with amplitude $2 q$ will therefore become

$$
\overline{\varepsilon\left(t_{0}\right) \varepsilon\left(t_{0}+\tau\right)}=\frac{q}{2 \Phi} \mathrm{e}^{-\Phi|\tau|} .
$$

By definition, the power spectral density (PSD, denoted by $\mathscr{S}$ ) is the Fourier transform of the autocorrelation function, hence

$$
\mathscr{S}(f)=\frac{q / \Phi^{2}}{1+(2 \pi f / \Phi)^{2}}=\frac{A_{0}}{1+\left(S_{t} / \phi_{\max }\right)^{2}},
$$

where $A_{0} \equiv q\left[L /\left(\bar{u}_{1} \phi\right)\right]^{2}, \phi_{\max } \equiv \phi /(2 \pi)$ and $S_{t}$ is the Strouhal number $\left(S_{t}=f L / \bar{u}_{1}\right)$. In general, one is interested in the wall-pressure PSD near the mean shock-foot position rather than the PSD of the shock-foot position itself. Let us assume that the instantaneous pressure at the mean shock-foot position $\bar{x}_{0}$ may be approximated by the mean pressure at $\bar{x}_{0}-\varepsilon$ :

$$
p_{w}\left(\bar{x}_{0}, t_{s}\right) \approx \bar{p}_{w}\left(\bar{x}_{0}-\varepsilon\right) .
$$

The above equation is not expected to be correct on fast time scales, hence the use of $t_{s}$, which was defined in (3.2). Since the shock motions in this study are considered small compared with the interaction length, one can expand (6.5a) using the first term in $\varepsilon$ :

$$
p_{w}\left(\bar{x}_{0}, t_{s}\right) \approx \bar{p}_{w}\left(\bar{x}_{0}\right)-\left.\frac{\mathrm{d} \bar{p}}{\mathrm{~d} x}\right|_{\bar{x}_{0}} \varepsilon
$$


For the slow time scales considered here, we have $p\left(t_{s}\right)-\bar{p} \approx \tilde{p}$; hence

$$
\tilde{p}_{w}\left(\bar{x}_{0}, t_{s}\right) \approx-\left.\frac{\mathrm{d} \bar{p}_{w}}{\mathrm{~d} x}\right|_{\bar{x}_{0}} \varepsilon .
$$

Therefore, the autocorrelation of the pressure fluctuations near the mean shock-foot position can be approximated by the shock-foot autocorrelation function using

$$
\overline{\tilde{p}_{w}\left(\bar{x}_{0}, t_{0}\right) \tilde{p}_{w}\left(\bar{x}_{0}, t_{0}+\tau\right)} \approx\left(\left.\frac{\mathrm{d} \bar{p}_{w}}{\mathrm{~d} x}\right|_{\bar{x}_{0}}\right)^{2} \overline{\varepsilon\left(t_{0}\right) \varepsilon\left(t_{0}+\tau\right)},
$$

for sufficiently large time lags $\tau$. Then, the wall-pressure PSD near the mean shockfoot position, denoted by $\mathscr{S}_{p}$, is

$$
\mathscr{S}_{p}\left(S_{t}\right) \approx \frac{A_{0}\left(\mathrm{~d} \bar{p}_{w} /\left.\mathrm{d} x\right|_{\bar{x}_{0}}\right)^{2}}{1+\left(S_{t} / \phi_{\max }\right)^{2}} .
$$

\subsection{Solution for forcing by synthetic turbulence}

It is emphasised that (6.7) is the response of the model to white-noise forcing and that the solution is valid only for sufficiently low frequencies (typically, $S_{t}<1$ ). As an alternative, in the event that the forcing term $C_{f_{0}}^{\prime \prime}$ is known, one can numerically integrate (6.2a). In practice, this may not be needed and (6.7) may be sufficiently accurate. To convince ourselves, we will use an artificial signal for $C_{f_{0}}^{\prime \prime}$, representative of the incoming turbulence. To do so, one can employ a digital filter approach, similar to that used to generate the inflow conditions for the LES. In this case, the problem is one-dimensional, and starting from $N$ normally distributed random numbers $\left\{a_{n}\right\}_{0 \leqslant n \leqslant N-1}$ with zero mean and unit variance (i.e. $\overline{a_{n}}=0, \overline{a_{n} a_{n}}=1$ and $\overline{a_{n} a_{m}}=0$ if $\left.n \neq m\right)$, the following synthetic turbulence series is produced:

$$
\begin{aligned}
& C_{f_{0}}^{\prime \prime}\left(t_{0}+n \Delta t\right)=C_{f_{0}}^{\prime \prime}\left(t_{0}+(n-1) \Delta t\right) \exp \left(-\frac{\pi \Delta t}{2 \tau_{c}}\right)+a_{n} \sqrt{q\left(1-\exp \left(-\frac{\pi \Delta t}{\tau_{c}}\right)\right)}, \\
& C_{f_{0}}^{\prime \prime}\left(t_{0}\right)=a_{0} \sqrt{q} \text { and }, n \in\{1, \ldots, N-1\},
\end{aligned}
$$

where $q$ is the imposed variance of $C_{f_{0}}^{\prime \prime}$ (i.e. $q=\overline{C_{f_{0}}^{\prime \prime} C_{f_{0}}^{\prime \prime}}$ ) and $\tau_{c}$ is the characteristic time scale of the correlation. In the present case, we take $q \approx 7 \times 10^{-4}, \tau_{c}=5 \times 10^{-2} \bar{u}_{1} / L$, $\Delta t=5 \times 10^{-3} \bar{u}_{1} / L$ and $N=5 \times 10^{8}$.

Once the synthetic signal $C_{f_{0}}^{\prime \prime}$ is obtained using (6.8), $(6.1 a)$ is integrated numerically using a fourth-order Runge-Kutta method, giving $\varepsilon(t)$, which is then used to compute $\mathscr{S}(f)$. If the wall-pressure PSD near the mean shock-foot position is of interest, the pressure-gradient conversion factor may be applied (see (6.6)). Note that for the weighted spectra, the correction is not necessary since the pressure-gradient factor term will appear in both the numerator and denominator. This equivalence between the shock-displacement and wall-pressure weighted spectra will be used to directly compare the model predictions with the experimental and numerical results, which are based on the pressure rather than the shock displacement itself. 

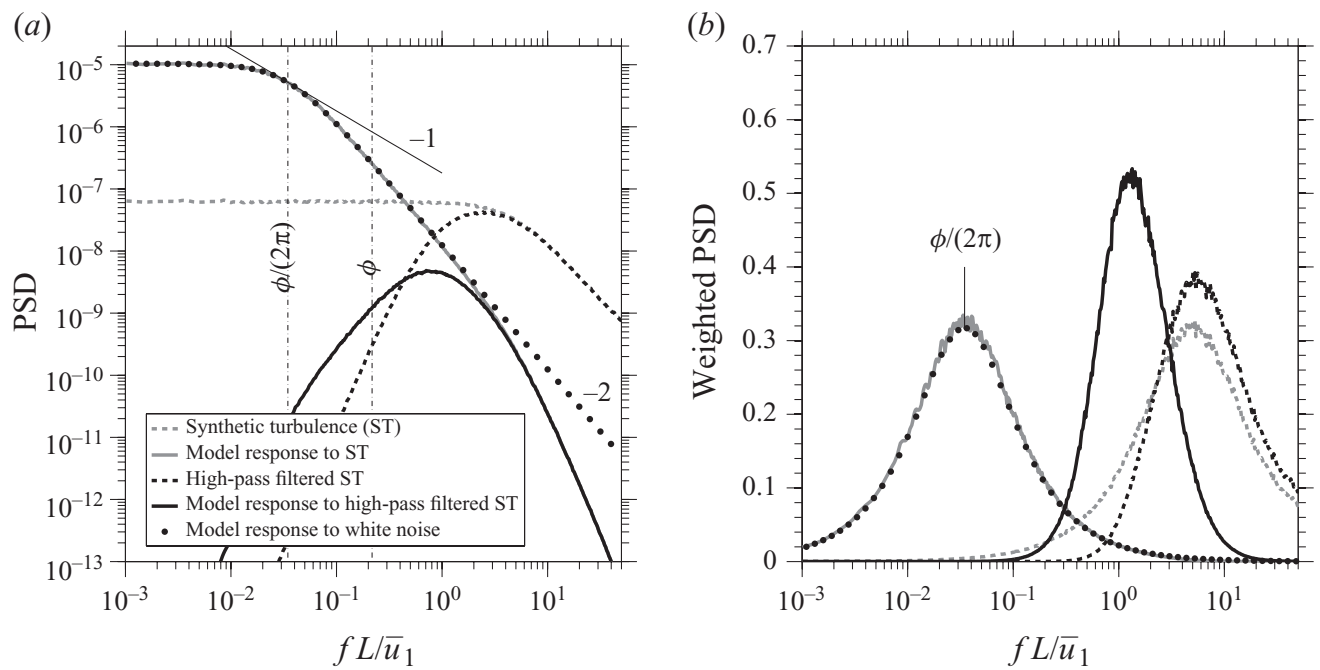

FIGURE 14. $(a, b)$ PSD and weighted PSD from the stochastic ODE using different forcing: a synthetic turbulence signal based on a one-dimensional digital filter approach (see $\S 6.3$ ), the same synthetic turbulence signal but high-pass filtered with cutoff frequency $f_{c}=5 \bar{u}_{1} / L$, and white noise (see $\S 6.2$ ).

\section{Model performance and discussion}

\subsection{Model results compared with LES and experimental findings}

Before comparing the predicted spectrum with experiment and LES, it is important to consider the effect of the choice of forcing as this may have implications for the discussion. First, the analytical solution to white noise (see $\S 6.2$ ) is considered. Second, the synthetic turbulence signal described in $\S 6.3$ is used to integrate the governing equation (6.1a). Finally, a high-pass filtered version of the same synthetic turbulence signal is examined. In all cases, the flow conditions correspond to the IUSTI $8^{\circ}$ case. Results are reported in figure 14.

First of all, it is seen that in the case of the white-noise and synthetic-turbulence forcing, the resulting shock motions exhibit significantly amplified low-frequency motions whereas the contributions of the higher frequencies are weaker than in the forcing itself. Moreover, it is found that at low frequencies the analytical solution to white noise is identical to the response to the synthetic-turbulence forcing. This is due to the particular synthetic-turbulence spectrum resembling that of white noise at low frequencies (see figure 14a). These results suggest that the model is not sensitive to the high-frequency content of the forcing, but to whether or not a level of noise is present at low frequencies. To test this idea, the synthetic turbulence was high-pass filtered to remove the low-frequency noise. As a consequence, the low-frequency motions disappear and the high-frequency content is reduced compared to the level of the forcing. Therefore, one important property of the system is that it acts as a low-pass filter. As such, it does not transfer energy from the higher to the lower frequencies but simply damps any fluctuations greater than a cutoff frequency while it amplifies any fluctuations smaller than this cutoff frequency. In fact, this is clear from (6.4), and figure 14 simply provides numerical evidence of the low-pass filtering property of $(6.1 a)$. 


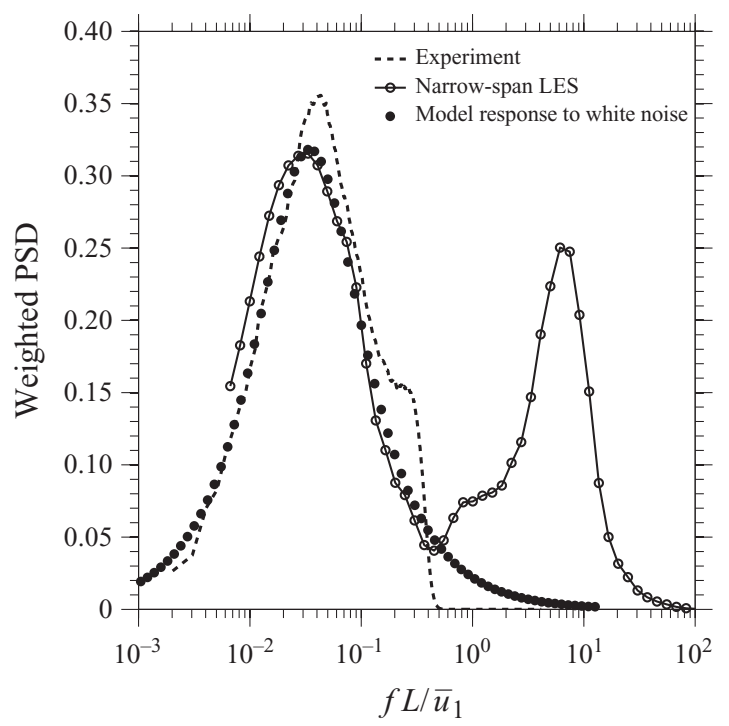

FIGURE 15. Weighted spectra from the model compared with the LES and experimental results. The LES spectrum is taken from figure 17 in Touber \& Sandham $(2009 b)$ and the experimental data from Dupont et al. (2006). Both the LES and experimental data are from wall-pressure probes near the mean shock-foot position. The experimental signal was low-pass filtered with cutoff frequency $f_{c}=2.5 \bar{u}_{1} / L$. The premultiplied spectra were normalised using the power available at low frequencies only (i.e. for the LES signal, the peak at higher frequencies is not included in the normalisation).

From (6.4) or (6.7), it is straightforward to see that the system is a first-order low-pass filter with cutoff Strouhal number $\phi /(2 \pi)$. The PSD of the shock motions or wall-pressure fluctuations near the mean shock foot rolls off as $S_{t}^{-2}$. Moreover, considering the premultiplied spectra (i.e. $f \times \mathscr{S}(f)$ ), one can show that there exists a maximum at $\phi /(2 \pi)$, which will be denoted by $\phi_{\max }$. Note that it coincides with the frequency (i.e. Strouhal number here) where the PSD is tangent to $S_{t}^{-1}$, as indicated in figure $14(a)$. This is the frequency typically quoted when characterising the property of the low-frequency oscillations (see e.g. Dupont et al. 2006). Incidentally, it corresponds to the cutoff frequency of the dynamical system (6.1).

On the basis of the above results, it appears justified to simply use a white-noise forcing to predict the wall-pressure weighted spectra and directly compare the result with the low-frequency motions observed both numerically and experimentally. Such a comparison is provided in figure 15, where the model is seen to be capable of predicting reasonably well not only the frequency of the most energetic low-frequency motion but also the broadband nature of the dynamics, which is an important aspect of the problem. Note that, in principle, the model can also predict the absolute spectral amplitude of the shock-foot displacement provided that the interaction length and the variance of the white noise are known. This encouraging result and the implications for understanding the underlying source of the low-frequency motions will be discussed in $\S 7.3$. Before doing so, we would like to take advantage of the model to describe the map of $\phi_{\max }$ for any given combination of upstream Mach number and wedge angle and discuss the sensitivity of the model to the choice of the constant values. 
(a)

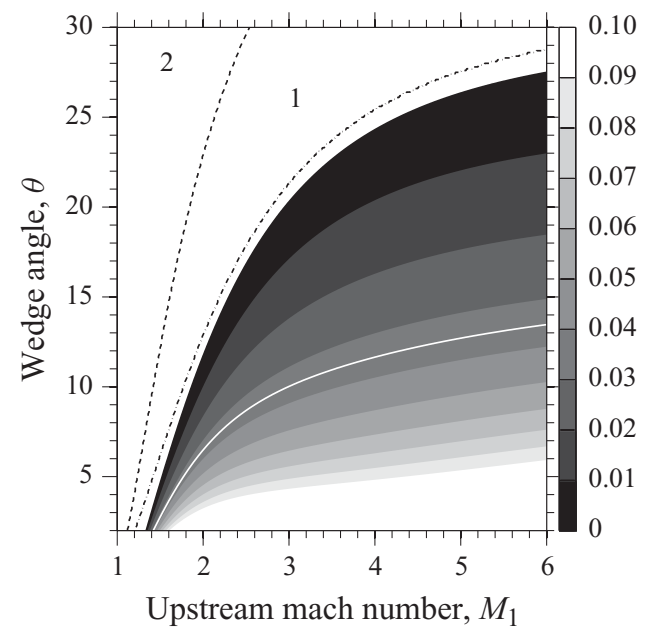

(b)

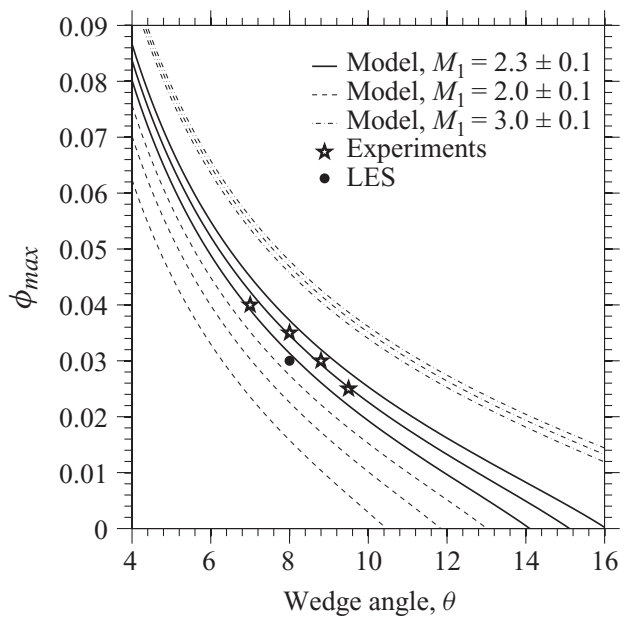

FIGURE 16. Predicted most energetic low frequency $\phi_{\max }$ for different $\left(M_{1}, \theta\right)$ pairs. In $(a)$, which is the map of $\phi /(2 \pi)$, the solid white line gives the $\phi_{\max }=0.035$ contour. The dashed line and dash-dotted line delimit two regions, labelled 1 and 2. Region 1 corresponds to Mach reflection cases and region 2 to cases where no oblique incident shock is formed. In $(b)$, the Mach 2.3 case of IUSTI is described for a wide range of wedge angles. The cases where $M_{1}=2$ and $M_{1}=3$ are also provided. For all cases, a variation of \pm 0.1 in the upstream Mach number value is applied to look at the sensitivity of the result to $\bar{M}_{1}$. The narrow-span LES result is also indicated together with the experimental results of Dupont et al. (2006). In both $(a)$ and $(b)$, the boundary-layer skin-friction properties were those of the IUSTI flow case.

\subsection{Cutoff frequency map and sensitivity to the model constants}

One great advantage of the present model is the possibility of using it for any given values of $M_{1}$ and $\theta$. Figure 16(a) shows the map of $\phi_{\max }$ for $M_{1}$ ranging from 1 to 6 and $\theta$ from $2^{\circ}$ to $30^{\circ}$, whenever a regular reflection exists. The first remark is that most values are within the range $10^{-2}$ to $10^{-1}$, which is consistent with the experimental observations of SBLI (see Dussauge, Dupont \& Debiève 2006, for example). Additionally, it is found that for a constant wedge angle, $\phi_{\max }$ increases with increasing Mach number and for a constant upstream Mach number, $\phi_{\max }$ decreases with increasing wedge angle. The latter trend can be tested against the experimental results of Dupont et al. (2006), as shown in figure $16(b)$. The agreement is well within the model and measurement uncertainties.

Note that $\phi_{\max }$ is expected to depend on the boundary-layer properties. Indeed, $\phi$ is explicitly related to the boundary-layer skin friction in $(6.1 c)$ and this could affect the results presented in figure $16(a)$, where $\bar{C}_{f_{0}}=1.35 \times 10^{-3}$. In addition, the modelling constants $F^{\prime}(0), r, r^{\prime}, r^{\prime \prime}$ and $\Lambda$ may all have significant impacts on the map of $\phi_{\max }$. To estimate the relative sensitivity to each of those constants, the map shown in figure $16(a)$ was computed with each constant successively doubled and halved (see Touber 2010). Overall, the monotonic trends are found to be preserved with steeper/more gradual slopes and/or increased/reduced levels of $\phi_{\max }$.

Two important results arose from the sensitivity study. First, the sensitivity of the model to the mean boundary-layer properties is weak for $\bar{C}_{f_{0}}$ and insignificant for $r^{\prime \prime}$, suggesting that the map in figure $16(a)$ is a good estimate for other mean boundary-layer properties (as long as the hypotheses used to derive the model hold). The mean boundary-layer properties thus play a major role in setting the interaction 
length (see the steady-state equation $(4.22 a)$ ) but their effect on the final dynamical equation is only weak. Second, the accuracy of the model for $\kappa_{2}$ and to a lesser extent for $\kappa_{p}$ is crucial. While $r$ can be easily determined to relatively good accuracy (see $\S 5), r^{\prime}$ is the most critical aspect of the present model and further improvements could be sought in the future. Nevertheless, the overall monotonicity of the map of $\phi_{\max }$ and the order of magnitude of the predicted $\phi_{\max }$ are maintained even for these sensitive cases. This demonstrates that the Strouhal-number value for the most energetic low-frequency shock motions is robust, with values remaining below 0.1 for a wide range of configurations, as argued by Dussauge et al. (2006).

Finally, it is important to bear in mind that the model is based on an approximate form of the momentum integral equation which itself relies on four assumptions (see $\S 4.3$ ), among which two are of primary importance. First, the interaction must be sufficiently large for the shock-crossing point to be above the incoming boundary layer. Therefore, one does not expect the model to be correct for weak interactions (i.e. for the smallest $\left(p_{2}^{+}-p_{1}\right) / \tau_{w}$ values). Second, the interaction was considered to be two-dimensional. Thus, any large spanwise wrinkling of the shock is not considered. In both cases, it would be possible to extend the model and release those constraints but this has been left for future work. We now proceed to a more general discussion about the contribution of the model to the understanding of the low-frequency shock motions.

\subsection{Discussion and implications with respect to the low-frequency unsteadiness}

Plotkin (1975), in the context of the compression-corner configuration, first postulated that the reflected shock could obey a stochastically forced first-order ordinary equation which is mathematically identical to that derived here (see $(6.1 a)$ ). The fact that the above lengthy derivations lead to the same governing equation as that proposed by Plotkin (1975) is comforting given the completely different approaches undertaken. In the present approach, the final governing equation is derived from the Navier-Stokes equations. To some extent, it may be seen as a proof of Plotkin's postulate (although this was not our original intention), with two distinct advantages that the underlying assumptions may be more clearly identified and that the time scale of the restoring mechanism is formally expressed as a function of the problem input parameters. In Plotkin (1975), one needs to determine this constant experimentally, resulting in a lack of applicability of the model, despite its mathematically appealing form. To the best of our knowledge, since the original publication of Plotkin (1975), only the two papers by Poggie \& Smits $(2001,2005)$ offer careful comparisons between Plotkin's model and experimental data, in each case with success.

Poggie \& Smits (2001) argue that, although the final model is described by a linear equation, it does not mean that the nonlinearities of the system are not accounted for. Their argument is that if one had considered a linearised theory (i.e. linearised Euler equations), the shock-motion spectrum would be the same as that of the incoming turbulence, which is not the case in the model. In the present derivation, one can see that, while the governing equation for the shock motions was clearly linearised (see steps between (4.19) and (4.21)) on the basis of sufficiently small shock displacements relatively to the interaction size, other significant nonlinear effects are mechanically embedded in the time scale $\phi^{-1}$. Indeed, looking at the constituents of $\phi$, one can see that although the model is expressed in the form of point-particle dynamics (i.e. the shock foot position), it does not convey a direct relation between a given velocity fluctuation and the shock response to it, as linearised Euler would do, but instead it accounts for integrated effects by means of the different thicknesses (see $(4.2 a-d)$ ), 
which are nonlinear functions of the velocity perturbations. In other words, the model accounts for the nonlinear coupling between the shock system and the boundary layer.

As mentioned in the previous section, the model describes this coupled shock/boundary-layer system as a low-pass filter with characteristic time scale $\phi^{-1}$. One remarkable result is that this time scale is significantly larger than any characteristic time scales of the incoming boundary layer $\left(\phi /(2 \pi)\right.$ is in the $10^{-2}$ to $10^{-1}$ range, giving $t_{s} \sim 10$ to $100 L / \bar{u}_{1}$ to compare with $t_{f}=\delta_{0} / \bar{u}_{1} \sim L / \bar{u}_{1}$ assuming that the interaction length scales with $\delta_{0}$ ). This conforms to experimental observations (Dupont et al. 2006), and the known issue in numerical simulations that such flows have long initial transients, even for laminar cases (indeed, in the absence of forcing, the convergence to the steady solution would be as $\exp \left(-t / t_{s}\right)$ ).

The low-pass filtering property of the system indicates that, strictly speaking, no transfer of energy from the higher to the lower frequencies is occurring. Instead, any high frequency is damped and any low frequency is amplified, with the frontier between high and low being determined by $\phi$. This was shown mathematically through the model response to white-noise forcing and numerically through direct integration of the response to synthetic turbulence signals. Therefore, the system itself is simply amplifying existing low-frequency fluctuations, even if energetically insignificant, while it filters out the high-frequency fluctuations, even if energetically significant. Moreover, the resulting broadband spectrum about a particular Strouhal number is not a property of the forcing but a characteristic of the shock/boundarylayer system itself.

On the basis of the preceding discussion, it is inferred that the origin of the low-frequency oscillations is not in the forcing but in the dynamics of the system formed by the shock/boundary-layer interaction. Of course, if one applies any specific forcing below the natural frequency of the system, such forcing will be picked up and magnified. A specific forcing could be any significantly long upstream coherent structures (see Ganapathisubramani et al. 2007, 2009, and references therein) or particular flow features within the interaction itself (see Pirozzoli \& Grasso 2006; Dussauge \& Piponniau 2008; Piponniau et al. 2009, and references therein). However, we stress that, mathematically speaking, these are not necessary and the lowfrequency motions can simply arise from a background (white) noise, as successfully demonstrated in figure 15.

\section{Conclusions}

The main contribution of this paper is the derivation of a stochastic ODE for the reflected-shock-foot motions, starting from the Navier-Stokes equations and based on some assumptions that were checked using LES data. The general form (4.16) of the governing equation relies on the assumed existence of a separation of variables (4.8), which is well supported by the LES data, allowing a transformation of what was initially a partial differential equation into an ordinary one. The derivation assumes two-dimensional motions (i.e. the spanwise wrinkling of the shock was not considered) with the shock-crossing point located above the incoming boundarylayer height $\delta_{0}$. Under such conditions, (4.16) was derived and then linearised on the basis of sufficiently small shock displacements and the analysis of LES data. This final form of the governing equation was found to be mathematically identical to the one postulated by Plotkin (1975) and capable of reproducing the wall-pressure low-frequency spectrum in the vicinity of the mean shock-foot position. 
Upon modelling the constituents of the derived governing equation, the dynamical system could be closed and expressed in terms of its input parameters: the upstream Mach number $M_{1}$, the wedge angle $\theta$ and the upstream boundary-layer properties (i.e. skin friction and momentum thickness). Although the upstream boundary-layer properties are found to be important for setting up the interaction length, the dynamical system was shown to be mainly controlled by $M_{1}$ and $\theta$. A wide range of input $\left(M_{1}, \theta\right)$ pairs were tested and the predicted most energetically significant low-frequency motions, expressed in the form of the Strouhal number $S_{t}$, were shown to remain in the range 0.01 to 0.1 , confirming the experimental evidence collected in Dussauge et al. (2006). The most energetic Strouhal number was found to increase with increasing $M_{1}$ for a constant wedge angle $\theta$, whereas it decreased with increasing wedge angle for constant $M_{1}$.

Mathematically speaking, the derived governing equation was shown to correspond to a first-order low-pass filter, and the analytical spectrum derived from forcing the system with white noise was shown to be in excellent agreement with the available experimental and numerical spectra. This result is consistent with the findings of Plotkin (1975) and Poggie \& Smits $(2001,2005)$ and leads to the suggestion that the low-frequency motions observed in SBLI need not be a characteristic of the forcing but simply the result of the low-pass filtering property of the dynamical system formed by the coupling between the boundary layer and the reflected shock, as demonstrated by the white-noise forcing. This does not mean that specific forcing from upstream (see Ganapathisubramani et al. 2007, amongst others) or downstream (see Pirozzoli \& Grasso 2006; Robinet 2007; Piponniau et al. 2009; Touber \& Sandham 2009b) does not play a role, but that they are not necessary. Obviously, if present and acting below the system cutoff frequency, they will inevitably be picked up by the system.

While further improvements to the proposed model are clearly possible (e.g. to include the shock spanwise wrinkling, derive a better model for $\kappa_{2}$, extend the model to compression ramps and/or hot/cold walls), it is interesting to comment on the implications of this work to efforts in controlling this flow. On the basis of the above discussion, there is no reason to believe that a periodic excitation could be of any help in inhibiting the low-frequency motions. From the dynamical-system point of view, there are two possible approaches: remove any low-frequency content in the forcing or modify the natural frequency of the system. The first option seems rather impracticable and it is probably better to focus on the second option. Obviously, one cannot modify the Navier-Stokes equations and a practical possibility is to modify the boundary conditions. Thus, one would need to implement wall-boundary conditions such that the net effect on the coupled shock/boundary-layer system may also be written in the form of a first-order linear ODE with ideally the same characteristic frequency as the natural system but with opposite sign. Supposing that such implementation is possible, the control system would then need to act on time scales of the order of $10 \delta_{0} / \bar{u}_{1}$, with the potential risk of exciting the system's natural frequency.

The authors would like to acknowledge the UK Turbulence Consortium EP/D044073/1 for the computational time provided on the HECToR facilities, the UK's national high-performance computing service, which is provided by EPCC at the University of Edinburgh and by CCLRC Daresbury Laboratory, and funded by the Office of Science and Technology through EPSRC's High End Computing Program. We are also grateful to the University of Southampton for the access to its high-performance computer, Iridis 2 . In addition, we would like to acknowledge the 
financial support of the European Union through the Sixth Framework Program with the UFAST project. Finally, we are grateful to J.-P. Dussauge and his co-workers at IUSTI for kindly making their data available and to O. Blesbois at Imperial College London for his time and advice when writing the mathematical developments presented in the appendices.

\section{Appendix A. Proof of the phase- and conditional-average relationships inherited from hypothesis 1}

\section{A.1. Proof of the first corollary}

Starting from the triple decomposition (3.3a), invoking the linear and scalarconserving properties of the conditional-averaging operator and assuming that $\left\langle u_{i}^{\prime \prime}\right\rangle_{\varepsilon_{0}, \Delta \sigma}=0$, it is straightforward to write

$$
\left\langle u_{i}\right\rangle_{\varepsilon_{0}, \Delta \sigma}=\frac{1}{\mathscr{N}\left(\mathscr{A}_{\varepsilon_{0}, \Delta \sigma}\right)} \int_{\mathscr{A}_{\varepsilon_{0}, \Delta \sigma}}\left(\bar{u}_{i}+\tilde{u}_{i}+u_{i}^{\prime \prime}\right) \mathrm{d} t=\left\langle\tilde{u}_{i}\right\rangle_{\varepsilon_{0}, \Delta \sigma}+\bar{u}_{i} .
$$

By introducing hypothesis 1 (see (3.4)) into the above equation, one finds

$$
\left\langle u_{i}\right\rangle_{\varepsilon_{0}, \Delta \sigma}-\bar{u}_{i}=\frac{1}{\mathscr{N}\left(\mathscr{A}_{\varepsilon_{0}, \Delta \sigma}\right)} \int_{\mathscr{A}_{\varepsilon_{0}, \Delta \sigma}} \tilde{u}_{i}\left(t_{s}\right) \mathrm{d} t_{s}=\frac{1}{\mathscr{N}\left(\mathscr{A}_{\varepsilon_{0}, \Delta \sigma}\right)} \int_{\mathscr{A}_{\varepsilon_{0}, \Delta \sigma}} \tilde{u}_{i}\left(\varepsilon\left(t_{s}\right)\right) \mathrm{d} t_{s} .
$$

Noting that the above integration is performed on the set $\mathscr{A}_{\varepsilon_{0}, \Delta \sigma}$, by definition of $\mathscr{A}_{\varepsilon_{0}, \Delta \sigma}$ one can write

$$
\varepsilon\left(t_{s}\right)=\varepsilon_{0}+\varphi\left(t_{s}\right) \Delta \sigma
$$

where $\varphi$ is a bounded function in $[0,1]$. If the phase-fluctuation velocity field $\tilde{u}_{i}(\varepsilon)$ is continuously differentiable on $\left[\varepsilon_{0}, \varepsilon_{0}+\Delta \sigma\right]$, Taylor's theorem with the Lagrange form of the remainder may be written as

$$
\begin{aligned}
\tilde{u}_{i}\left(\varepsilon\left(t_{s}\right)\right)=\tilde{u}_{i}\left(\varepsilon_{0}\right) & +\Delta \sigma q_{0}\left(t_{s}\right), \\
& \text { with }\left|q_{0}\left(t_{s}\right)\right| \leqslant \varphi\left(t_{s}\right) \sup _{\varepsilon \in\left[\varepsilon_{0}, \varepsilon_{0}+\Delta \sigma\right]}\left|\frac{\partial \tilde{u}_{i}}{\partial \varepsilon}\right| \leqslant \sup _{\varepsilon \in\left[\varepsilon_{0}, \varepsilon_{0}+\Delta \sigma\right]}\left|\frac{\partial \tilde{u}_{i}}{\partial \varepsilon}\right| .
\end{aligned}
$$

The condition that $\tilde{u}_{i}(\varepsilon)$ be continuously differentiable is questionable due to the presence of shocks, but this is purely a mathematical concern here, since the numerical velocity fields are differentiated across shockwaves in the process of solving the Navier-Stokes equations. Thus, we argue that in practice the remainder is well defined and bounded. Next, (A 4) is used in the integral (A 2):

$$
\left\langle u_{i}\right\rangle_{\varepsilon_{0}, \Delta \sigma}-\bar{u}_{i}=\frac{1}{\mathscr{N}\left(\mathscr{A}_{\varepsilon_{0}, \Delta \sigma}\right)}\left[\int_{\mathscr{A}_{\varepsilon_{0}, \Delta \sigma}} \tilde{u}_{i}\left(\varepsilon_{0}\right) \mathrm{d} t_{s}+\int_{\mathscr{A}_{\varepsilon_{0}, \Delta \sigma}} \Delta \sigma q_{0}\left(t_{s}\right) \mathrm{d} t_{s}\right] .
$$

The first integral is trivial since the integrand does not depend on $t_{s}$. Moreover, given the definition of $\mathscr{N}\left(\mathscr{A}_{\varepsilon_{0}, \Delta \sigma}\right)$ in $(3.1 b)$, the first term is simply $\tilde{u}_{i}\left(\varepsilon_{0}\right)$. The second integral concerns a bounded function of $t_{s} \in \mathscr{A}_{\varepsilon_{0}, \Delta \sigma}$ and is therefore controlled by the supremum of the function times the integral range. Hence

$$
\left\langle u_{i}\right\rangle_{\varepsilon_{0}, \Delta \sigma}-\bar{u}_{i}=\tilde{u}_{i}\left(\varepsilon_{0}\right)+\Delta \sigma Q_{0}\left(\mathscr{A}_{\varepsilon_{0}, \Delta \sigma}\right), \quad \text { with }\left|Q_{0}\left(\mathscr{A}_{\varepsilon_{0}, \Delta \sigma}\right)\right| \leqslant \sup _{\varepsilon \in\left[\varepsilon_{0}, \varepsilon_{0}+\Delta \sigma\right]}\left|\frac{\partial \tilde{u}_{i}}{\partial \varepsilon}\right| .
$$


For simplicity, (A 6) can be expressed in the Landau notation by noting that the remainder is of the order of $\Delta \sigma$ :

$$
\left\langle u_{i}\right\rangle_{\varepsilon_{0}, \Delta \sigma}-\bar{u}_{i}=\tilde{u}_{i}\left(\varepsilon_{0}\right)+O(\Delta \sigma) .
$$

In words, (A 7) simply expresses the idea that on $\mathscr{A}_{\varepsilon_{0}, \Delta \sigma}$, the phase-fluctuation field $\tilde{u}_{i}$ may be approximated by $\left\langle u_{i}\right\rangle_{\varepsilon_{0}, \Delta \sigma}-\bar{u}_{i}$ with an error of the order of $\Delta \sigma$, provided that hypothesis 1 is satisfied.

\section{A.2. Proof of the second corollary}

For the second corollary, we still consider that the phase-fluctuation velocity field is continuously differentiable on $\left[\varepsilon_{0}, \varepsilon_{0}+\Delta \sigma\right]$ and therefore start from (A 4) to write that for $t_{s} \in \mathscr{A}_{\varepsilon_{0}, \Delta \sigma}$ :

$$
\begin{aligned}
\tilde{u}_{i}\left(\varepsilon\left(t_{s}\right)\right) \tilde{u}_{j}\left(\varepsilon\left(t_{s}\right)\right) & =\left[\tilde{u}_{i}\left(\varepsilon_{0}\right)+\Delta \sigma q_{0}\left(t_{s}\right)\right]\left[\tilde{u}_{j}\left(\varepsilon_{0}\right)+\Delta \sigma s_{0}\left(t_{s}\right)\right] \\
\text { with }\left|q_{0}\left(t_{s}\right)\right| & \leqslant \sup _{\varepsilon \in\left[\varepsilon_{0}, \varepsilon_{0}+\Delta \sigma\right]}\left|\frac{\partial \tilde{u}_{i}}{\partial \varepsilon}\right|,\left|s_{0}\left(t_{s}\right)\right| \leqslant \sup _{\varepsilon \in\left[\varepsilon_{0}, \varepsilon_{0}+\Delta \sigma\right]}\left|\frac{\partial \tilde{u}_{j}}{\partial \varepsilon}\right| .
\end{aligned}
$$

Equation (A 8) can then be integrated over $\mathscr{A}_{\varepsilon_{0}, \Delta \sigma}$ :

$$
\int_{\mathscr{A}_{\varepsilon_{0}, \Delta \sigma}} \tilde{u}_{i}\left(\varepsilon\left(t_{s}\right)\right) \tilde{u}_{j}\left(\varepsilon\left(t_{s}\right)\right) \mathrm{d} t_{s}=\int_{\mathscr{A}_{\varepsilon_{0}, \Delta \sigma}}\left[\tilde{u}_{i}\left(\varepsilon_{0}\right)+\Delta \sigma q_{0}\left(t_{s}\right)\right]\left[\tilde{u}_{j}\left(\varepsilon_{0}\right)+\Delta \sigma s_{0}\left(t_{s}\right)\right] \mathrm{d} t_{s} .
$$

Terms $\tilde{u}_{i}\left(\varepsilon_{0}\right), \tilde{u}_{j}\left(\varepsilon_{0}\right)$ being independent of $t_{s}$, and $q_{0}\left(t_{s}\right), s_{0}\left(t_{s}\right)$ being bounded functions on $\mathscr{A}_{\varepsilon_{0}, \Delta \sigma}$, (A 9) becomes

$$
\begin{aligned}
& \int_{\mathscr{A}_{\varepsilon_{0}, \Delta \sigma}} \tilde{u}_{i}\left(\varepsilon\left(t_{s}\right)\right) \tilde{u}_{j}\left(\varepsilon\left(t_{s}\right)\right) \mathrm{d} t_{s}=\left[\tilde{u}_{i}\left(\varepsilon_{0}\right) \tilde{u}_{j}\left(\varepsilon_{0}\right)+\Delta \sigma\left\{Q_{0}\left(\mathscr{A}_{\varepsilon_{0}, \Delta \sigma}\right) \tilde{u}_{j}\left(\varepsilon_{0}\right)\right.\right. \\
& \left.\left.\quad+S_{0}\left(\mathscr{A}_{\varepsilon_{0}, \Delta \sigma}\right) \tilde{u}_{i}\left(\varepsilon_{0}\right)\right\}+\Delta \sigma^{2} M_{0}\right] \mathscr{N}\left(\mathscr{A}_{\varepsilon_{0}, \Delta \sigma}\right), \\
& \quad \text { with }\left|Q_{0}\left(\mathscr{A}_{\varepsilon_{0}, \Delta \sigma}\right)\right| \leqslant \sup _{\varepsilon \in\left[\varepsilon_{0}, \varepsilon_{0}+\Delta \sigma\right]}\left|\frac{\partial \tilde{u}_{i}}{\partial \varepsilon}\right|, \quad\left|S_{0}\left(\mathscr{A}_{\varepsilon_{0}, \Delta \sigma}\right)\right| \leqslant \sup _{\varepsilon \in\left[\varepsilon_{0}, \varepsilon_{0}+\Delta \sigma\right]}\left|\frac{\partial \tilde{u}_{j}}{\partial \varepsilon}\right| \\
& \quad \text { and }\left|M_{0}\right| \leqslant \sup _{\varepsilon \in\left[\varepsilon_{0}, \varepsilon_{0}+\Delta \sigma\right]}\left|\frac{\partial \tilde{u}_{i}}{\partial \varepsilon}\right| \sup _{\varepsilon \in\left[\varepsilon_{0}, \varepsilon_{0}+\Delta \sigma\right]}\left|\frac{\partial \tilde{u}_{j}}{\partial \varepsilon}\right| .
\end{aligned}
$$

Using Landau's notation, the second corollary can be expressed as

$$
\int_{\mathscr{A}_{\varepsilon_{0}, \Delta \sigma}} \tilde{u}_{i}\left(\varepsilon\left(t_{s}\right)\right) \tilde{u}_{j}\left(\varepsilon\left(t_{s}\right)\right) \mathrm{d} t_{s}=\left[\tilde{u}_{i}\left(\varepsilon_{0}\right) \tilde{u}_{j}\left(\varepsilon_{0}\right)+O(\Delta \sigma)\right] \mathscr{N}\left(\mathscr{A}_{\varepsilon_{0}, \Delta \sigma}\right) .
$$

\section{A.3. Estimation of the phase-fluctuation stress tensor}

Starting with the stress tensor computed from the conditionally averaged fields,

$$
I=\frac{1}{T} \sum_{k=0}^{N-1}\left[\left\langle u_{i}\right\rangle_{\varepsilon_{\min }+k \Delta \sigma, \Delta \sigma}-\bar{u}_{i}\right]\left[\left\langle u_{j}\right\rangle_{\varepsilon_{\min }+k \Delta \sigma, \Delta \sigma}-\bar{u}_{j}\right] \mathscr{N}\left(\mathscr{A}_{\varepsilon_{\min }+k \Delta \sigma, \Delta \sigma}\right),
$$

where $N=\left(\varepsilon_{\max }-\varepsilon_{\min }\right) / \Delta \sigma$ with $\varepsilon_{\max }=\max (\varepsilon(t)), \varepsilon_{\min }=\min (\varepsilon(t)), t \in[0, T]$; it is possible to use the first corollary to write

$$
\begin{aligned}
I=\frac{1}{T} \sum_{k=0}^{N-1}\left[\tilde{u}_{i}\left(\varepsilon_{\min }+k \Delta \sigma\right)+\Delta\right. & \left.\sigma Q_{0}\left(\mathscr{A}_{\varepsilon_{\min }+k \Delta \sigma, \Delta \sigma}\right)\right]\left[\tilde{u}_{j}\left(\varepsilon_{\min }+k \Delta \sigma\right)\right. \\
& \left.+\Delta \sigma S_{0}\left(\mathscr{A}_{\varepsilon_{\min }+k \Delta \sigma, \Delta \sigma}\right)\right] \mathscr{N}\left(\mathscr{A}_{\varepsilon_{\min }+k \Delta \sigma, \Delta \sigma}\right),
\end{aligned}
$$


and re-arrange to

$$
\begin{aligned}
I= & \frac{1}{T} \sum_{k=0}^{N-1}\left[\tilde{u}_{i}\left(\varepsilon_{\text {min }}+k \Delta \sigma\right) \tilde{u}_{j}\left(\varepsilon_{\text {min }}+k \Delta \sigma\right)+\Delta \sigma\left\{Q_{0}\left(\mathscr{A}_{\varepsilon_{\min }+k \Delta \sigma, \Delta \sigma}\right) \tilde{u}_{j}\left(\varepsilon_{\text {min }}+k \Delta \sigma\right)\right.\right. \\
& \left.+S_{0}\left(\mathscr{A}_{\varepsilon_{\min }+k \Delta \sigma, \Delta \sigma}\right) \tilde{u}_{i}\left(\varepsilon_{\min }+k \Delta \sigma\right)\right\} \\
& \left.+\Delta \sigma^{2} Q_{0}\left(\mathscr{A}_{\varepsilon_{\min }+k \Delta \sigma, \Delta \sigma}\right) S_{0}\left(\mathscr{A}_{\varepsilon_{\min }+k \Delta \sigma, \Delta \sigma}\right)\right] \mathscr{N}\left(\mathscr{A}_{\varepsilon_{\min }+k \Delta \sigma, \Delta \sigma}\right) .
\end{aligned}
$$

The form (A 10) of the second corollary is introduced in (A 14) to find

$$
I=\frac{1}{T} \sum_{k=0}^{N-1} \int_{\mathscr{A}_{\varepsilon_{\min }+k \Delta \sigma, \Delta \sigma}} \tilde{u}_{i}\left(\varepsilon\left(t_{s}\right)\right) \tilde{u}_{j}\left(\varepsilon\left(t_{s}\right)\right) \mathrm{d} t_{s}+J,
$$

where

$$
\begin{aligned}
J= & \frac{1}{T} \sum_{k=0}^{N-1} \Delta \sigma^{2}\left\{Q_{0}\left(\mathscr{A}_{\varepsilon_{\min }+k \Delta \sigma, \Delta \sigma}\right) S_{0}\left(\mathscr{A}_{\varepsilon_{\min }+k \Delta \sigma, \Delta \sigma}\right)\right. \\
& \left.-M_{0}\left(\mathscr{A}_{\varepsilon_{\text {min }}+k \Delta \sigma, \Delta \sigma}\right)\right\} \mathscr{N}\left(\mathscr{A}_{\varepsilon_{\min }+k \Delta \sigma, \Delta \sigma}\right) .
\end{aligned}
$$

Since the functions $Q_{0}, S_{0}, M_{0}$ are all bounded, the sum $J$ can be crudely bounded by

$$
J \leqslant \frac{\Delta \sigma^{2}}{T} M \sum_{k=0}^{N-1} \mathscr{N}\left(\mathscr{A}_{\varepsilon_{\min }+k \Delta \sigma, \Delta \sigma}\right)
$$

with $M$ a constant taken to be

$$
\begin{aligned}
M= & \max _{k \in\{0, \ldots, N-1\}}\left[\sup _{\mathscr{A}_{\varepsilon_{\min }+k \Delta \sigma, \Delta \sigma}}\left|Q_{0}\left(\mathscr{A}_{\varepsilon_{\min }+k \Delta \sigma, \Delta \sigma}\right) S_{0}\left(\mathscr{A}_{\varepsilon_{\min }+k \Delta \sigma, \Delta \sigma}\right)\right|\right. \\
& \left.+\sup _{\mathscr{A}_{\varepsilon_{\min }+k \Delta \sigma, \Delta \sigma}}\left|M_{0}\left(\mathscr{A}_{\varepsilon_{\min }+k \Delta \sigma, \Delta \sigma}\right)\right|\right] .
\end{aligned}
$$

By definition of $\mathscr{N}$, the sum on all $\mathscr{N}\left(\mathscr{A}_{\varepsilon_{\min }+k \Delta \sigma, \Delta \sigma}\right)$ is simply equal to $T$, so that

$$
I=\frac{1}{T} \sum_{k=0}^{N-1} \int_{\mathscr{A}_{\varepsilon_{\min }+k \Delta \sigma, \Delta \sigma}} \tilde{u}_{i}\left(\varepsilon\left(t_{s}\right)\right) \tilde{u}_{j}\left(\varepsilon\left(t_{s}\right)\right) \mathrm{d} t_{s}+K \Delta \sigma^{2}, \text { with } K \leqslant M .
$$

Invoking the additivity properties of the integral, the sum over all the subsets $\mathscr{A}_{\varepsilon_{\min }+k \Delta \sigma, \Delta \sigma}$ of $[0, T]$ can be changed into the integral over their union:

$$
I=\frac{1}{T} \int_{\bigcup_{k=0}^{N-1} \mathscr{A}_{\varepsilon_{\min }+k \Delta \sigma, \Delta \sigma}} \tilde{u}_{i}\left(\varepsilon\left(t_{s}\right)\right) \tilde{u}_{j}\left(\varepsilon\left(t_{s}\right)\right) \mathrm{d} t_{s}+K \Delta \sigma^{2}
$$

Given the definition of $N$, the union of the subsets $\mathscr{A}_{\varepsilon_{\min }+k \Delta \sigma, \Delta \sigma}$ spans the full time interval $[0, T]$ only once, so that (A 20) becomes, using Landau's notation,

$$
I=\underbrace{\frac{1}{T} \int_{T} \tilde{u}_{i}\left(\varepsilon\left(t_{s}\right)\right) \tilde{u}_{j}\left(\varepsilon\left(t_{s}\right)\right) \mathrm{d} t_{s}}_{\tilde{\tilde{u}_{i}} \tilde{u}_{j}}+O\left(\Delta \sigma^{2}\right) .
$$


Therefore, to second order in $\Delta \sigma$, we have

$$
\overline{\tilde{u}_{i} \tilde{u}_{j}} \approx \frac{1}{T} \sum_{k=0}^{N-1}\left[\left\langle u_{i}\right\rangle_{\varepsilon_{\min }+k \Delta \sigma, \Delta \sigma}-\bar{u}_{i}\right]\left[\left\langle u_{j}\right\rangle_{\varepsilon_{\min }+k \Delta \sigma, \Delta \sigma}-\bar{u}_{j}\right] \mathscr{N}\left(\mathscr{A}_{\varepsilon_{\min }+k \Delta \sigma, \Delta \sigma}\right) .
$$

\section{Appendix B. Derivation of the momentum integral equation}

Let us start from the continuity equation and the streamwise component of the momentum equation:

$$
\begin{aligned}
\frac{\partial \rho}{\partial t}+\frac{\partial \rho u}{\partial x}+\frac{\partial \rho v}{\partial y}+\frac{\partial \rho w}{\partial z} & =0 \\
\rho \frac{\partial u}{\partial t}+\rho u \frac{\partial u}{\partial x}+\rho v \frac{\partial u}{\partial y}+\rho w \frac{\partial u}{\partial z} & =-\frac{\partial p}{\partial x}+\frac{1}{R e}\left[\frac{\partial \tau_{x x}}{\partial x}+\frac{\partial \tau_{x y}}{\partial y}+\frac{\partial \tau_{x z}}{\partial z}\right] \\
\tau_{i j} & =\mu\left(\frac{\partial u_{j}}{\partial x_{i}}+\frac{\partial u_{i}}{\partial x_{j}}-\frac{2}{3} \delta_{i j} \frac{\partial u_{k}}{\partial x_{k}}\right) .
\end{aligned}
$$

Equation (B $1 b)$ can be integrated in the wall-normal direction up to $h$ (see figure 5 for the notations):

$$
\begin{aligned}
\int_{0}^{h}\left(\rho \frac{\partial u}{\partial t}+\rho u \frac{\partial u}{\partial x}+\rho w \frac{\partial u}{\partial z}\right) & \mathrm{d} y+\underbrace{\int_{0}^{h} \rho v \frac{\partial u}{\partial y} \mathrm{~d} y}_{A} \\
& =\int_{0}^{h}\left(-\frac{\partial p}{\partial x}+\frac{1}{R e}\left[\frac{\partial \tau_{x x}}{\partial x}+\frac{\partial \tau_{x y}}{\partial y}+\frac{\partial \tau_{x z}}{\partial z}\right]\right) \mathrm{d} y .
\end{aligned}
$$

Term $A$ can be integrated by parts and together with (B $1 a)$ gives

$$
\begin{aligned}
A=[\rho u v]_{0}^{h}-\int_{0}^{h} u \frac{\partial \rho v}{\partial y} \mathrm{~d} y=-u_{h} \int_{0}^{h}\left(\frac{\partial \rho}{\partial t}\right. & \left.+\frac{\partial \rho u}{\partial x}+\frac{\partial \rho w}{\partial z}\right) \mathrm{d} y \\
& +\int_{0}^{h} u\left(\frac{\partial \rho}{\partial t}+\frac{\partial \rho u}{\partial x}+\frac{\partial \rho w}{\partial z}\right) \mathrm{d} y
\end{aligned}
$$

where the no-slip boundary condition at $y=0$ was used to eliminate $\left.\rho u v\right|_{y=0}$. Equation (B 2) can be re-written, using (B 3):

$$
\begin{aligned}
& \underbrace{\int_{0}^{h} \rho \frac{\partial u}{\partial t} \mathrm{~d} y-u_{h} \int_{0}^{h} \frac{\partial \rho}{\partial t} \mathrm{~d} y+\int_{0}^{h} u \frac{\partial \rho}{\partial t} \mathrm{~d} y}_{a}+\underbrace{\int_{0}^{h} \rho u \frac{\partial u}{\partial x} \mathrm{~d} y-u_{h} \int_{0}^{h} \frac{\partial \rho u}{\partial x} \mathrm{~d} y+\int_{0}^{h} u \frac{\partial \rho u}{\partial x} \mathrm{~d} y}_{b} \\
& +\underbrace{\int_{0}^{h} \rho w \frac{\partial u}{\partial z} \mathrm{~d} y-u_{h} \int_{0}^{h} \frac{\partial \rho w}{\partial z} \mathrm{~d} y+\int_{0}^{h} u \frac{\partial \rho w}{\partial z} \mathrm{~d} y}_{c} \\
& =\underbrace{\int_{0}^{h}-\frac{\partial p}{\partial x} \mathrm{~d} y}_{d}+\underbrace{\frac{1}{R e} \int_{0}^{h} \frac{\partial \tau_{x y}}{\partial y} \mathrm{~d} y}_{e}+\underbrace{\frac{1}{R e} \int_{0}^{h} \frac{\partial \tau_{x x}}{\partial x} \mathrm{~d} y}_{f}+\underbrace{\frac{1}{R e} \int_{0}^{h} \frac{\partial \tau_{x z}}{\partial z} \mathrm{~d} y}_{g} .
\end{aligned}
$$


To re-arrange terms $a$ to $g$, we have to commute the integration and derivation operators. To do so, we recall Leibniz's rule

$$
\frac{\partial}{\partial \alpha}\left[\int_{0}^{h} f \mathrm{~d} y\right]=\int_{0}^{h} \frac{\partial f}{\partial \alpha} \mathrm{d} y+f(y=h) \frac{\partial h}{\partial \alpha}-f(y=0) \underbrace{\frac{\partial 0}{\partial \alpha}} .
$$

Term $a$ can be re-arranged using Leibniz's rule:

$$
\begin{aligned}
a=\int_{0}^{h} \frac{\partial \rho u}{\partial t} \mathrm{~d} y-u_{h} \int_{0}^{h} \frac{\partial \rho}{\partial y} \mathrm{~d} y= & \frac{\partial}{\partial t}\left[\int_{0}^{h} \rho u \mathrm{~d} y\right]-\rho_{h} u_{h} \frac{\partial h}{\partial t} \\
& -u_{h}\left(\frac{\partial}{\partial t}\left[\int_{0}^{h} \rho \mathrm{d} y\right]-\rho_{h} \frac{\partial h}{\partial t}\right) \\
= & \frac{\partial}{\partial t}\left[\int_{0}^{h} \rho u \mathrm{~d} y\right]-u_{h} \frac{\partial}{\partial t}\left[\int_{0}^{h} \rho \mathrm{d} y\right] .
\end{aligned}
$$

Equation (4.2a) can be re-arranged:

$$
\rho_{h} u_{h} \delta_{1}=\rho_{h} u_{h} \int_{0}^{h}\left(1-\frac{\rho u}{\rho_{h} u_{h}}\right) \mathrm{d} y=\int_{0}^{h} \rho_{h} u_{h}\left(1-\frac{\rho u}{\rho_{h} u_{h}}\right) \mathrm{d} y=\rho_{h} u_{h} h-\int_{0}^{h} \rho u \mathrm{~d} y,
$$

where the independence of $\rho_{h}$ and $u_{h}$ on $y\left(u_{h}=u_{h}(x, z, t)\right)$ was used. The same manipulation can be performed with $(4.2 d)$ and term $a$ becomes

$$
a=\rho_{h}\left(h-\delta_{1}\right) \frac{\partial u_{h}}{\partial t}+u_{h} \frac{\partial}{\partial t}\left[\rho_{h}\left(\delta_{\rho}-\delta_{1}\right)\right] .
$$

Similarly, term $b$ is re-arranged using Leibniz's rule:

$$
\begin{aligned}
b=\int_{0}^{h} \frac{\partial \rho u u}{\partial x} \mathrm{~d} y-u_{h} \int_{0}^{h} \frac{\partial \rho u}{\partial x} \mathrm{~d} y= & \frac{\partial}{\partial x}\left[\int_{0}^{h} \rho u u \mathrm{~d} y\right]-\rho_{h} u_{h} u_{h} \frac{\partial h}{\partial x} \\
& -u_{h}\left(\frac{\partial}{\partial x}\left[\int_{0}^{h} \rho u \mathrm{~d} y\right]-\rho_{h} u_{h} \frac{\partial h}{\partial x}\right) \\
= & \frac{\partial}{\partial x}\left[\int_{0}^{h} \rho u u \mathrm{~d} y\right]-u_{h} \frac{\partial}{\partial x}\left[\int_{0}^{h} \rho u \mathrm{~d} y\right] .
\end{aligned}
$$

From $(4.2 b)$, one finds

$$
\begin{aligned}
\rho_{h} u_{h}^{2} \delta_{2}=\rho_{h} u_{h}^{2} \int_{0}^{h} \frac{\rho u}{\rho_{h} u_{h}}\left(1-\frac{u}{u_{h}}\right) \mathrm{d} y=u_{h} \int_{0}^{h} \rho u \mathrm{~d} y-\int_{0}^{h} \rho u u \mathrm{~d} y & \\
& =\rho_{h} u_{h}^{2}\left(h-\delta_{1}\right)-\int_{0}^{h} \rho u u \mathrm{~d} y
\end{aligned}
$$

so that $b$ becomes

$$
b=\rho_{h} u_{h}\left(h-\delta_{1}\right) \frac{\partial u_{h}}{\partial x}-\frac{\partial}{\partial x}\left[\rho_{h} u_{h}^{2} \delta_{2}\right]
$$


Invoking Leibniz's rule again, $c$ is rearranged:

$$
\begin{aligned}
c=\int_{0}^{h} \frac{\partial \rho u w}{\partial z} \mathrm{~d} y-u_{h} \int_{0}^{h} \frac{\partial \rho w}{\partial z} \mathrm{~d} y= & \frac{\partial}{\partial z}\left[\int_{0}^{h} \rho u w \mathrm{~d} y\right]-\rho_{h} u_{h} w_{h} \frac{\partial h}{\partial z} \\
& -u_{h}\left(\frac{\partial}{\partial z}\left[\int_{0}^{h} \rho w \mathrm{~d} y\right]-\rho_{h} w_{h} \frac{\partial h}{\partial z}\right) \\
= & \frac{\partial}{\partial z}\left[\int_{0}^{h} \rho u w \mathrm{~d} y\right]-u_{h} \frac{\partial}{\partial z}\left[\int_{0}^{h} \rho w \mathrm{~d} y\right] .
\end{aligned}
$$

Note that $h$ is, by construction, allowed to depend on $z$ but is independent of $x$, so that term $d$ becomes

$$
d=-\left(\frac{\partial}{\partial x}\left[\int_{0}^{h} p \mathrm{~d} y\right]-p_{h} \frac{\partial h}{\partial x}\right)=-\frac{\partial}{\partial x}\left[\int_{0}^{h} p \mathrm{~d} y\right] .
$$

From $(4.2 c)$, noting that $p_{h}$ does not depend on $y$, one finds

$$
p_{h} \delta_{p}=p_{h} \int_{0}^{h}\left(1-\frac{p}{p_{h}}\right) \mathrm{d} y=p_{h} h-\int_{0}^{h} p \mathrm{~d} y
$$

so that

$$
d=\frac{\partial}{\partial x}\left[p_{h}\left(\delta_{p}-h\right)\right]
$$

Term $e$ can be easily integrated to give (using the definition (4.3))

$$
e=\frac{1}{R e}\left\{\left[\mu\left(\frac{\partial v}{\partial x}+\frac{\partial u}{\partial y}\right)\right]_{y=h}-\left[\mu \frac{\partial u}{\partial y}\right]_{y=0}\right\}=\frac{\mu_{h}}{R e}\left(\left.\frac{\partial v}{\partial x}\right|_{y=h}+\left.\frac{\partial u}{\partial y}\right|_{y=h}\right)-\frac{1}{2} C_{f} \rho_{h} u_{h}^{2} .
$$

Terms $f$ and $g$ are rearranged, using Leibniz's rule and the independence of $h$ on $x$ :

$$
\begin{gathered}
f=\frac{1}{R e}\left(\frac{\partial}{\partial x}\left[\int_{0}^{h} \tau_{x x} \mathrm{~d} y\right]-\left.\tau_{x x}\right|_{y=h} \frac{\partial h}{\partial x}\right)=\frac{1}{R e} \frac{\partial}{\partial x}\left[\int_{0}^{h} \tau_{x x} \mathrm{~d} y\right], \\
g=\frac{1}{\operatorname{Re}}\left(\frac{\partial}{\partial z}\left[\int_{0}^{h} \tau_{x z} \mathrm{~d} y\right]-\left.\tau_{x z}\right|_{y=h} \frac{\partial h}{\partial z}\right) .
\end{gathered}
$$

Using (B 8) to (B 18) in (B 4) leads to (4.1):

$$
\begin{aligned}
& \rho_{h}\left(h-\delta_{1}\right)\left[\frac{\partial u_{h}}{\partial t}+u_{h} \frac{\partial u_{h}}{\partial x}\right]+u_{h} \frac{\partial}{\partial t}\left[\rho_{h}\left(\delta_{\rho}-\delta_{1}\right)\right]-\frac{\partial}{\partial x}\left[\rho_{h} u_{h}^{2} \delta_{2}\right]+\frac{\partial}{\partial z}\left[\int_{0}^{h} \rho u w \mathrm{~d} y\right] \\
& -u_{h} \frac{\partial}{\partial z}\left[\int_{0}^{h} \rho w \mathrm{~d} y\right]=\frac{\partial}{\partial x}\left[p_{h}\left(\delta_{p}-h\right)\right]+\frac{\mu_{h}}{R e}\left[\left.\frac{\partial v}{\partial x}\right|_{y=h}+\left.\frac{\partial u}{\partial y}\right|_{y=h}\right]-\frac{1}{2} C_{f} \rho_{h} u_{h}^{2} \\
& +\frac{1}{R e} \frac{\partial}{\partial x}\left[\int_{0}^{h} \tau_{x x} \mathrm{~d} y\right]+\frac{1}{R e}\left(\frac{\partial}{\partial z}\left[\int_{0}^{h} \tau_{x z} \mathrm{~d} y\right]-\left.\tau_{x z}\right|_{y=h} \frac{\partial h}{\partial z}\right) .
\end{aligned}
$$




\section{Appendix C. Series expansions of the oblique-shock relations}

\section{C.1. Expansion of $\sin ^{2}(\iota+\theta)$}

To derive the following series expansions in terms of $\eta / h_{0}$, the notations presented in figure 10 will be used. From this figure, one can write the geometrical relations:

$$
\begin{aligned}
\tan \alpha & =\frac{h_{0}}{l_{0}}, \\
\sin \iota & =\frac{h_{0}+\eta}{\sqrt{\left(h_{0}+\eta\right)^{2}+\left(l_{0}-\varepsilon+s\right)^{2}}}, \\
\cos \iota & =\frac{l_{0}-\varepsilon+s}{\sqrt{\left(h_{0}+\eta\right)^{2}+\left(l_{0}-\varepsilon+s\right)^{2}}} .
\end{aligned}
$$

From (4.12) and (4.13) it is straightforward to show that

$$
s-\varepsilon=\eta \underbrace{\left[\frac{1-k}{k \tan \beta}\right]}_{K_{0}} .
$$

From trigonometric identities, one can write

$$
\sin ^{2}(\iota+\theta)=\sin ^{2} \iota \cos ^{2} \theta+\cos ^{2} \iota \sin ^{2} \theta+\sin \iota \cos \iota \sin (2 \theta) .
$$

Using $(\mathrm{C} 1 b, c)$ and $(\mathrm{C} 2)$,

$$
\begin{aligned}
\sin ^{2}(\iota+\theta)= & \frac{1}{h_{0}^{2}+\eta^{2}+2 h_{0} \eta+l_{0}^{2}+K_{0}^{2} \eta^{2}+2 l_{0} K_{0} \eta}\left\{\left(h_{0}^{2}+\eta^{2}+2 h_{0} \eta\right) \cos ^{2} \theta\right. \\
& \left.+\left(l_{0}^{2}+K_{0}^{2} \eta^{2}+2 l_{0} K_{0} \eta\right) \sin ^{2} \theta+\left(h_{0} l_{0}+h_{0} K_{0} \eta+l_{0} \eta+K_{0} \eta^{2}\right) \sin (2 \theta)\right\} .
\end{aligned}
$$

Upon multiplying both the numerator and denominator of the right-hand side of (C $3 b$ ) by $1 / h_{0}^{2}$ and defining $q \equiv \eta / h_{0}$, (C $3 b$ ) becomes

$$
\begin{aligned}
\sin ^{2}(\iota+\theta)= & \frac{1}{1+q^{2}+2 q+\left(l_{0} / h_{0}\right)^{2}+K_{0}^{2} q^{2}+2\left(l_{0} / h_{0}\right) K_{0} q}\left\{\left(1+q^{2}+2 q\right) \cos ^{2} \theta\right. \\
& +\left(\left(l_{0} / h_{0}\right)^{2}+K_{0}^{2} q^{2}+2\left(l_{0} / h_{0}\right) K_{0} q\right) \sin ^{2} \theta \\
& \left.+\left(l_{0} / h_{0}+K_{0} q+\left(l_{0} / h_{0}\right) q+K_{0} q^{2}\right) \sin (2 \theta)\right\} .
\end{aligned}
$$

Substituting ( $\mathrm{C} 1 a)$ into the above equation gives

$$
\begin{aligned}
\sin ^{2}(\iota+\theta)= & \frac{1}{1+q^{2}+2 q+1 / \tan ^{2} \alpha+K_{0}^{2} q^{2}+2 K_{0} q / \tan \alpha}\left\{\left(1+q^{2}+2 q\right) \cos ^{2} \theta\right. \\
& +\left(1 / \tan ^{2} \alpha+K_{0}^{2} q^{2}+2 K_{0} q / \tan \alpha\right) \sin ^{2} \theta \\
& \left.+\left(1 / \tan \alpha+K_{0} q+q / \tan \alpha+K_{0} q^{2}\right) \sin (2 \theta)\right\}
\end{aligned}
$$

Factorising $(\mathrm{C} 3 d)$ by $1 /\left(1+1 / \tan ^{2} \alpha\right)$ and noting that $\sin ^{2} \alpha=1 /\left(1+1 / \tan ^{2} \alpha\right)$, one finds

$$
\begin{aligned}
\sin ^{2}(\iota+\theta)= & \frac{\sin ^{2} \alpha}{1+2 C_{1} q+C_{2} q^{2}}\left\{\left(1+2 q+q^{2}\right) \cos ^{2} \theta\right. \\
& +\left(1+2 K_{1} q+K_{1}^{2} q^{2}\right) \sin ^{2} \theta / \tan ^{2} \alpha \\
& \left.+\left(1+q\left(1+K_{1}\right)+K_{1} q^{2}\right) \sin (2 \theta) / \tan \alpha\right\}
\end{aligned}
$$


where $C_{1} \equiv\left(K_{1}+\tan ^{2} \alpha\right) /\left(1+\tan ^{2} \alpha\right), C_{2} \equiv\left(K_{1}^{2}+\tan ^{2} \alpha\right) /\left(1+\tan ^{2} \alpha\right)$ and $K_{1} \equiv K_{0} \tan \alpha$. If the study is restricted to shock oscillations such that $q \ll 1$, one can expand the fractional term, i.e.

$$
\frac{1}{1+2 C_{1} q+C_{2} q^{2}}=1-2 C_{1} q+\left(4 C_{1}^{2}-C_{2}\right) q^{2}+O\left(q^{3}\right)
$$

Using $(\mathrm{C} 4)$ in $(\mathrm{C} 3 e)$ and retaining only terms up to the first order in $q$, one finds

$$
\begin{aligned}
\sin ^{2}(\iota+\theta)= & \sin ^{2} \alpha\left\{\left[1+2 q\left(1-C_{1}\right)\right] \cos ^{2} \theta+\left[1+2 q\left(K_{1}-C_{1}\right)\right] \sin ^{2} \theta / \tan ^{2} \alpha\right. \\
& \left.+\left[1+q\left(1+K_{1}-2 C_{1}\right)\right] \sin (2 \theta) / \tan \alpha\right\}+O\left(q^{2}\right),
\end{aligned}
$$

which upon regrouping terms of similar orders gives

$$
\begin{aligned}
\sin ^{2}(\iota+\theta)= & \overbrace{\sin ^{2} \alpha \cos ^{2} \theta+\cos ^{2} \alpha \sin ^{2} \theta+\sin (2 \theta) \sin \alpha \cos \alpha}^{\sin ^{2}(\alpha+\theta)} \\
& +\left\{2\left(1-C_{1}\right) \cos ^{2} \theta \sin ^{2} \alpha+2\left(K_{1}-C_{1}\right) \sin ^{2} \theta \cos ^{2} \alpha\right. \\
& \left.+\left(1+K_{1}-2 C_{1}\right) \sin (2 \theta) \sin \alpha \cos \alpha\right\} q+O\left(q^{2}\right) .
\end{aligned}
$$

It is relatively straightforward to see that $K_{1}-C_{1}=\sin ^{2} \alpha\left(K_{1}-1\right)$ and $1-C_{1}=(1-$ $\left.K_{1}\right) \cos ^{2} \alpha$, which if combined gives $1+K_{1}-2 C_{1}=\left(1-K_{1}\right) \cos (2 \alpha)$ using the relation $\cos (2 \alpha)=\cos ^{2} \alpha-\sin ^{2} \alpha$. On the basis of those remarks and after few manipulations of trigonometric identities, (C $5 b$ ) simplifies to

$$
\sin ^{2}(\iota+\theta)=\sin ^{2}(\alpha+\theta)+\left\{\frac{1-K_{1}}{2} \sin (2 \alpha) \sin (2 \alpha+2 \theta)\right\} q+O\left(q^{2}\right) .
$$

Using the definitions of $K_{1}$ and $K_{0},(\mathrm{C} 5 c)$ becomes

$$
\begin{aligned}
\sin ^{2}(\iota+\theta)= & \sin ^{2}(\alpha+\theta) \\
& +\frac{1}{2}\left[1+\frac{\tan \alpha}{\tan \beta}\left(1-\frac{1}{k}\right)\right] \sin (2 \alpha) \sin (2 \alpha+2 \theta) q+O\left(q^{2}\right) .
\end{aligned}
$$

If we now assume that $k$ can be modelled according to (5.4), (C 5d) becomes

$$
\begin{gathered}
\sin ^{2}(\iota+\theta)=\sin ^{2}(\alpha+\theta)+\frac{1}{2} \varkappa(\alpha, \beta, \theta) q+O\left(q^{2}\right), \text { with } \\
\varkappa(\alpha, \beta, \theta)=\frac{\tan \alpha+\tan \beta}{\tan \beta(1-1 / \tan \alpha)-1} \sin (2 \alpha) \sin [2(\alpha+\theta)] . \\
\text { C.2. Expansion of } p_{3} / p_{1}
\end{gathered}
$$

The pressure in region 3 varies according to

$$
\frac{p_{3}}{p_{1}}=\frac{p_{2}^{+}}{p_{1}}\left\{1+\frac{2 \gamma}{1+\gamma}\left[M_{2}^{2} \sin ^{2}(\iota+\theta)-1\right]\right\} .
$$

Using (C6), (C 7) can be expanded as

$$
\frac{p_{3}}{p_{1}}=\frac{p_{2}^{+}}{p_{1}}\left\{1+\frac{2 \gamma}{1+\gamma}\left[M_{2}^{2}\left(\sin ^{2}(\alpha+\theta)+\frac{1}{2} \varkappa(\alpha, \beta, \theta) \frac{\eta}{h_{0}}\right)-1\right]\right\}+O\left(\frac{\eta^{2}}{h_{0}^{2}}\right)
$$

where the mean pressure in region 3 (i.e. $\bar{p}_{3}$ ) can be identified:

$$
\frac{p_{3}}{p_{1}}=\frac{\bar{p}_{3}}{p_{1}}+\frac{p_{2}^{+}}{p_{1}} \frac{\gamma}{1+\gamma} M_{2}^{2} \varkappa(\alpha, \beta, \theta) \frac{\eta}{h_{0}}+O\left(\frac{\eta^{2}}{h_{0}^{2}}\right) \text {. }
$$


Hence

$$
\frac{p_{3}}{p_{1}}=\frac{\bar{p}_{3}}{p_{1}}+A \frac{\eta}{h_{0}}+O\left(\frac{\eta^{2}}{h_{0}^{2}}\right) \text {, with } A=\frac{p_{2}^{+}}{p_{1}} \frac{\gamma \varkappa M_{2}^{2}}{1+\gamma}
$$

\section{C.3. Expansion of $\rho_{3} / \rho_{1}$}

The density in region 3 varies according to

$$
\frac{\rho_{3}}{\rho_{1}}=\frac{\rho_{2}^{+}}{\rho_{1}}\left\{\frac{(\gamma+1) M_{2}^{2} \sin ^{2}(\iota+\theta)}{2+(\gamma-1) M_{2}^{2} \sin ^{2}(\iota+\theta)}\right\} .
$$

Substituting (C 6) into (C 10) gives

$$
\frac{\rho_{3}}{\rho_{1}}=\frac{\rho_{2}^{+}}{\rho_{1}}\left[\frac{a_{0}}{b_{0}}+\frac{\gamma+1}{2 b_{0}} M_{2}^{2} \varkappa q+O\left(q^{2}\right)\right]\left[1+\frac{\gamma-1}{2 b_{0}} M_{2}^{2} \varkappa q+O\left(q^{2}\right)\right]^{-1},
$$

where $a_{0} \equiv(\gamma+1) M_{2}^{2} \sin ^{2}(\alpha+\theta)$, and $b_{0} \equiv 2+(\gamma-1) M_{2}^{2} \sin ^{2}(\alpha+\theta)$. The term with power -1 can be expanded, leading to

$$
\begin{aligned}
\frac{\rho_{3}}{\rho_{1}} & =\frac{\rho_{2}^{+}}{\rho_{1}}\left[\frac{a_{0}}{b_{0}}+\frac{\gamma+1}{2 b_{0}} M_{2}^{2} \varkappa q+O\left(q^{2}\right)\right]\left[1-\frac{\gamma-1}{2 b_{0}} M_{2}^{2} \varkappa q+O\left(q^{2}\right)\right], \\
& =\frac{\rho_{2}^{+}}{\rho_{1}} \frac{a_{0}}{b_{0}}\left[1-\frac{\gamma-1}{2 b_{0}} M_{2}^{2} \varkappa q+\frac{\gamma+1}{2 a_{0}} M_{2}^{2} \varkappa q\right]+O\left(q^{2}\right) .
\end{aligned}
$$

Noting that $a_{0} / b_{0}=\bar{\rho}_{3} / \rho_{2}^{+},(\mathrm{C} 11 c)$ can be written as

$$
\begin{aligned}
& \frac{\rho_{3}}{\rho_{1}}=\frac{\bar{\rho}_{3}}{\rho_{1}}+B \frac{\eta}{h_{0}}+O\left(\frac{\eta^{2}}{h_{0}^{2}}\right) \\
& \quad \text { with } B=x\left[\frac{1}{2 \sin ^{2}(\alpha+\theta)}-\frac{(\gamma-1) M_{2}^{2}}{4+2(\gamma-1) M_{2}^{2} \sin ^{2}(\alpha+\theta)}\right] \frac{\bar{\rho}_{3}}{\rho_{1}} .
\end{aligned}
$$

C.4. Expansion of $M_{3} / M_{1}$

The Mach number in region 3 is computed according to

$$
\frac{M_{3}}{M_{1}}=\frac{1}{M_{1} \sin \iota} \sqrt{\frac{1+(1 / 2)(\gamma-1) M_{2}^{2} \sin ^{2}(\iota+\theta)}{\gamma M_{2}^{2} \sin ^{2}(\iota+\theta)-(\gamma-1) / 2}} .
$$

Substituting $(\mathrm{C} 6)$ and denoting $\chi(\alpha, \beta, 0)$ by $\varkappa_{0}$, one finds

$$
\begin{aligned}
\frac{M_{3}}{M_{1}}= & \frac{1}{M_{1}}\left[\sin ^{2} \alpha+\frac{1}{2} \varkappa_{0} q+O\left(q^{2}\right)\right]^{-1 / 2}\left[1+\frac{\gamma-1}{2} M_{2}^{2}\left(\sin ^{2}(\alpha+\theta)+\frac{1}{2} \varkappa q+O\left(q^{2}\right)\right)\right]^{1 / 2} \\
& \times\left[\frac{1-\gamma}{2}+\gamma M_{2}^{2}\left(\sin ^{2}(\alpha+\theta)+\frac{1}{2} \varkappa q+O\left(q^{2}\right)\right)\right]^{-1 / 2} .
\end{aligned}
$$

Each terms in powers of $\pm 1 / 2$ can be expanded up to the first order:

$$
\begin{aligned}
\frac{M_{3}}{M_{1}}= & \frac{1}{M_{1}}\left[\frac{1}{\sin \alpha}-\frac{\varkappa_{0}}{4 \sin ^{3} \alpha} q+O\left(q^{2}\right)\right]\left[\sqrt{\frac{b_{0}}{2}}+\frac{(\gamma-1) M_{2}^{2} \varkappa}{8 \sqrt{b_{0} / 2}} q+O\left(q^{2}\right)\right] \\
& \times\left[\frac{1}{\sqrt{c_{0}}}-\frac{\gamma M_{2}^{2} \varkappa}{4 c_{0}^{3 / 2}} q+O\left(q^{2}\right)\right],
\end{aligned}
$$


where $b_{0}$ is the same as in $(\mathrm{C} 11 a)$ and $c_{0}=(1-\gamma) / 2+\gamma M_{2}^{2} \sin ^{2}(\alpha+\theta)$. Regrouping terms of similar orders in $q$, one finds

$$
\frac{M_{3}}{M_{1}}=\frac{\bar{M}_{3}}{M_{1}}\left\{1+\frac{1}{4}\left[\frac{(\gamma-1) M_{2}^{2} \varkappa}{b_{0}}-\frac{\gamma M_{2}^{2} \varkappa}{c_{0}}-\frac{\varkappa_{0}}{\sin ^{2} \alpha}\right] q\right\}+O\left(q^{2}\right),
$$

where $\bar{M}_{3}=\sqrt{b_{0} /\left(2 c_{0} \sin ^{2} \alpha\right)}$. Hence

$$
\begin{aligned}
& \frac{M_{3}}{M_{1}}=\frac{\bar{M}_{3}}{M_{1}}+C \frac{\eta}{h_{0}}+O\left(\frac{\eta^{2}}{h_{0}^{2}}\right), \\
& \text { with } C=\frac{\bar{M}_{3}}{M_{1}}\left\{x\left[\frac{(\gamma-1) M_{2}^{2}}{8+4(\gamma-1) M_{2}^{2} \sin ^{2}(\alpha+\theta)}-\frac{\gamma M_{2}^{2}}{2(1-\gamma)+4 \gamma M_{2}^{2} \sin ^{2}(\alpha+\theta)}\right]\right. \\
& \left.-\frac{(\tan \alpha+\tan \beta) \cos ^{2} \alpha}{\tan \beta(1-1 / \tan \alpha)-1}\right\} \text {. }
\end{aligned}
$$

\section{C.5. Expansion of $\rho_{3} u_{3}\left(1-u_{3} / u_{1}\right) /\left(\rho_{1} u_{1}\right)$}

To expand $\rho_{3} u_{3}\left(1-u_{3} / u_{1}\right) /\left(\rho_{1} u_{1}\right)$, let us first note that

$$
\frac{\rho_{3} u_{3}}{\rho_{1} u_{1}}\left(1-\frac{u_{3}}{u_{1}}\right)=\frac{M_{3}}{M_{1}}\left(\sqrt{\frac{\rho_{3} p_{3}}{\rho_{1} p_{1}}}-\frac{M_{3} p_{3}}{M_{1} p_{1}}\right) .
$$

Using (C9), (C 12) and (C 15), (C 16) becomes

$$
\begin{aligned}
\frac{\rho_{3} u_{3}}{\rho_{1} u_{1}}\left(1-\frac{u_{3}}{u_{1}}\right)= & {\left[\frac{\bar{M}_{3}}{M_{1}}+C q+O\left(q^{2}\right)\right]\left\{\left[\left(\frac{\bar{\rho}_{3}}{\rho_{1}}+B q+O\left(q^{2}\right)\right)\left(\frac{\bar{p}_{3}}{p_{1}}+A q+O\left(q^{2}\right)\right)\right]^{1 / 2}\right.} \\
& \left.-\left(\frac{\bar{M}_{3}}{M_{1}}+C q+O\left(q^{2}\right)\right)\left(\frac{\bar{p}_{3}}{p_{1}}+A q+O\left(q^{2}\right)\right)\right\} .
\end{aligned}
$$

Expanding the square root and the products inside the curly brackets leads to

$$
\begin{aligned}
\frac{\rho_{3} u_{3}}{\rho_{1} u_{1}}\left(1-\frac{u_{3}}{u_{1}}\right)= & {\left[\frac{\bar{M}_{3}}{M_{1}}+C q+O\left(q^{2}\right)\right]\left\{\sqrt{\frac{\bar{\rho}_{3} \bar{p}_{3}}{\rho_{1} p_{1}}}+\frac{1}{2} \sqrt{\frac{\rho_{1} p_{1}}{\bar{\rho}_{3} \bar{p}_{3}}}\left(\frac{\bar{\rho}_{3}}{\rho_{1}} A+\frac{\bar{p}_{3}}{p_{1}} B\right) q\right.} \\
& \left.-\frac{\bar{M}_{3} \bar{p}_{3}}{M_{1} p_{1}}-\left(\frac{\bar{M}_{3}}{M_{1}} A+\frac{\bar{p}_{3}}{p_{1}} C\right) q+O\left(q^{2}\right)\right\} .
\end{aligned}
$$

The last product is then expanded to give

$$
\begin{aligned}
\frac{\rho_{3} u_{3}}{\rho_{1} u_{1}}\left(1-\frac{u_{3}}{u_{1}}\right)= & \frac{\bar{M}_{3}}{M_{1}}\left(\sqrt{\frac{\bar{\rho}_{3} \bar{p}_{3}}{\rho_{1} p_{1}}}-\frac{\bar{M}_{3} \bar{p}_{3}}{M_{1} p_{1}}\right)+\frac{\bar{M}_{3}}{M_{1}}\left\{\frac{1}{2} \sqrt{\frac{p_{1} \bar{\rho}_{3}}{\bar{p}_{3} \rho_{1}}} A+\frac{1}{2} \sqrt{\frac{\rho_{1} \bar{p}_{3}}{\bar{\rho}_{3} p_{1}}} B\right. \\
& \left.-\frac{\bar{M}_{3}}{M_{1}} A-\frac{\bar{p}_{3}}{p_{1}} C+\frac{M_{1}}{\bar{M}_{3}} C\left(\sqrt{\frac{\bar{\rho}_{3} \bar{p}_{3}}{\rho_{1} p_{1}}}-\frac{\bar{M}_{3} \bar{p}_{3}}{M_{1} p_{1}}\right)\right\} q+O\left(q^{2}\right) .
\end{aligned}
$$

The first term in $(\mathrm{C} 17 c)$ is $\bar{\rho}_{3} \bar{u}_{3}\left(1-\bar{u}_{3} / u_{1}\right) /\left(\rho_{1} u_{1}\right)$, so that

$$
\begin{aligned}
\frac{\rho_{3} u_{3}}{\rho_{1} u_{1}}\left(1-\frac{u_{3}}{u_{1}}\right)= & \frac{\bar{\rho}_{3} \bar{u}_{3}}{\rho_{1} u_{1}}\left(1-\frac{\bar{u}_{3}}{u_{1}}\right)+\frac{\bar{M}_{3}}{M_{1}}\left\{\left(\frac{1}{2} \sqrt{\frac{p_{1} \bar{\rho}_{3}}{\bar{p}_{3} \rho_{1}}}-\frac{\bar{M}_{3}}{M_{1}}\right) A+\frac{1}{2} \sqrt{\frac{\rho_{1} \bar{p}_{3}}{\bar{\rho}_{3} p_{1}}} B\right. \\
& \left.+C\left(\frac{M_{1}}{\bar{M}_{3}} \sqrt{\frac{\bar{\rho}_{3} \bar{p}_{3}}{\rho_{1} p_{1}}}-2 \frac{\bar{p}_{3}}{p_{1}}\right)\right\} q+O\left(q^{2}\right) .
\end{aligned}
$$


Hence

$$
\begin{aligned}
& \frac{\rho_{3} u_{3}}{\rho_{1} u_{1}}\left(1-\frac{u_{3}}{u_{1}}\right)=\frac{\bar{\rho}_{3} \bar{u}_{3}}{\rho_{1} u_{1}}\left(1-\frac{\bar{u}_{3}}{u_{1}}\right)+D \frac{\eta}{h_{0}}+O\left(\frac{\eta^{2}}{h_{0}^{2}}\right), \\
& \text { with } D=\frac{\bar{M}_{3}}{M_{1}}\left\{\left(\frac{1}{2} \sqrt{\frac{p_{1} \bar{\rho}_{3}}{\bar{p}_{3} \rho_{1}}}-\frac{\bar{M}_{3}}{M_{1}}\right) A+\frac{1}{2} \sqrt{\frac{\rho_{1} \bar{p}_{3}}{\bar{\rho}_{3} p_{1}}} B+\left(\frac{M_{1}}{\bar{M}_{3}} \sqrt{\frac{\bar{\rho}_{3} \bar{p}_{3}}{\rho_{1} p_{1}}}-2 \frac{\bar{p}_{3}}{p_{1}}\right) C\right\},
\end{aligned}
$$

where $A, B$ and $C$ are defined in (C9), (C 12) and (C 15), respectively.

\section{REFERENCES}

Carpenter, M. H., Nordstrom, J. \& Gottlieb, D. 1998 A stable and conservative interface treatment of arbitrary spatial accuracy. Tech. Rep. CR-206921. NASA.

Ducros, F., Ferrand, V., Nicoud, F., Weber, C., Darrace, D., Gacherieu, C. \& Poinsot, T. 1999 Large-eddy simulation of the shock/turbulence interaction. J. Comput. Phys. 152, 517-549.

Dupont, P., Haddad, C. \& Debiève, J. F. 2006 Space and time organization in a shock-induced separated boundary layer. J. Fluid Mech. 559, 255-277.

Dussauge, J.-P., Dupont, P. \& Debiève, J.-F. 2006 Unsteadiness in shock wave boundary layer interaction with separation. Aerosp. Sci. Technol. 10, 85-91.

Dussauge, J.-P. \& PiponniaU, S. 2008 Shock/boundary-layer interactions: possible sources of unsteadiness. J. Fluids Struct. 24, 1166-1175.

Ganapathisubramani, B., Clemens, N. T. \& Dolling, D. S. 2007 Effects of upstream boundary layer on the unsteadiness of shock-induced separation. J. Fluid Mech. 585, 369-394.

Ganapathisubramani, B., Clemens, N. T. \& Dolling, D. S. 2009 Low-frequency dynamics of shock-induced separation in a compression ramp interaction. J. Fluid Mech. 585, 397-425.

GARNIER, E. 2009 Stimulated detached eddy simulation of three-dimensional shock/boundary layer interaction. Shock Waves 19 (6), 479-486.

Garnier, E., Sagaut, P. \& Deville, M. 2002 Large eddy simulation of shock/boundary-layer interaction. AIAA J. 40 (10), 1935-1944.

Humble, R. A., Elsinga, G. E., Scarana, F. \& van Oudheusden, B. W. 2009 Three-dimensional instantaneous structure of a shock wave/turbulent boundary layer interaction. J. Fluid Mech. 622, 33-62.

Inagaki, M., Kondoh, T. \& Nagano, Y. 2005 A mixed-time-scale SGS model with fixed modelparameters for practical LES. J. Fluids Engng 127, 1-13.

KLeIN, M., SADIKI, A. \& JANicKa, J. 2003 A digital filter based generation of inflow data for spatially developing direct numerical or large eddy simulations. J. Comput. Phys. 186, 652-665.

Moin, P., Squires, K., Савот, W. \& LeE, S. 1991 A dynamic subgrid-scale model for compressible turbulence and scalar transport. Phys. Fluids 3 (11), 2746-2757.

Piponniau, S., Dussauge, J.-P., Debiève, J.-F. \& Dupont, P. 2009 A simple model for low-frequency unsteadiness in shock-induced separation. J. Fluid Mech. 629, 87-108.

Pirozzoli, S. \& Grasso, F. 2006 Direct numerical simulation of impinging shock wave/turbulent boundary layer interaction at $M=2.25$. Phys. Fluids 18 (6), 065113.

Plotkin, K. J. 1975 Shock wave oscillation driven by turbulent boundary-layer fluctuations. AIAA J. 13 (8), 1036-1040.

Poggie, J. \& Smits, A. J. 2001 Shock unsteadiness in a reattaching shear layer. J. Fluid Mech. 429, $155-185$.

Poggie, J. \& Smits, A. J. 2005 Experimental evidence for Plotkin model of shock unsteadiness in separated flow. Phys. Fluids 17 (1), 018107.

Polivanov, P. A., Sidorenko, A. A. \& Maslov, A. A. 2009 Experimental study of unsteady effects in shock wave/turbulent boundary layer interaction. In 47th AIAA Aerospace Sciences Meeting, Orlando, Florida, USA.

Priebe, S., Wu, M. \& Martin, M. P. 2009 Direct numerical simulation of a reflected-shockwave/turbulent-boundary-layer interaction. AIAA J. 47 (5), 1174-1185.

Ringuette, M. J., Bookey, P., Wyckham, C. \& Smits, A. J. 2009 Experimental study of a Mach 3 compression ramp interaction at $\operatorname{Re}_{\theta}=2400$. AIAA J. 47 (2), 373-385. 
Risken, H. 1989 The Fokker-Plank Equation, 2nd edn. Springer.

RoBinET, J.-C. 2007 Bifurcations in shock-wave/laminar-boundary-layer interaction: global instability approach. J. Fluid Mech. 579, 85-112.

Sagaut, P. 2005 Large Eddy Simulations for Incompressible Flows, 3rd edn. Springer.

Sandham, N. D., LI, Q. \& YeE, H. C. 2002 Entropy splitting for high-order numerical simulation of compressible turbulence. J. Comput. Phys. 178, 307-322.

SAndhu, H. S. \& SAndham, N. D. 1994 Boundary conditions for spatially growing compressible shear layers. Tech. Rep. QMW-EP-1100. Queen Mary, University of London.

Thompson, K. W. 1987 Time dependent boundary conditions for hyperbolic systems. J. Comput. Phys. 68 (1), 1-24.

Touber, E. 2010 Unsteadiness in shock-wave/boundary-layer interactions. PhD thesis, University of Southampton, School of Engineering Sciences.

Touber, E. \& Sandham, N. D. 2008 Oblique shock impinging on a turbulent boundary layer: low-frequency mechanisms. In 38th Fluid Dynamics Conference, Seattle, Washington, USA. AIAA Paper 2008-4170.

Touber, E. \& SANDham, N. D. 2009a Comparison of three large-eddy simulations of shock-induced turbulent separation bubbles. Shock Waves 19 (6), 469-478.

Touber, E. \& SANDhAM, N. D. $2009 b$ Large-eddy simulation of low-frequency unsteadiness in a turbulent shock-induced separation bubble. Theor. Comput. Fluid Dyn. 23, 79-107.

Touber, E. \& SANDham, N. D. 2009c Large-eddy simulations of an oblique shock impinging on a turbulent boundary layer: effect of the spanwise confinement on the low-frequency oscillations. In 2nd Intl Conf. on Turbulence and Interactions, Sainte-Luce, Martinique.

Touber, E. \& SANDhAM, N. D. 2010 Oblique-shock/turbulent-boundary-layer interaction. arXiv:1010.2044v1. Available at: http://arxiv.org/abs/1010.2044v1.

White, F. M. 1991 Viscous Fluid Flow, 2nd edn. McGraw-Hill.

Wu, M. \& Martin, M. P. 2008 Analysis of shock motion in shockwave and turbulent boundary layer interaction using direct numerical simulation data. J. Fluid Mech. 594, 71-83.

Yee, H. C., Sandham, N. D. \& Djomehri, M. J. 1999 Low-dissipative high-order shock-capturing methods using characteristic-based filters. J. Comput. Phys. 150, 199-238. 